\author{
Aus dem Fachbereich Medizin \\ der Johann Wolfgang Goethe-Universität \\ Frankfurt am Main \\ betreut am \\ Gustav-Embden-Zentrum der Biochemie \\ Institut für Biochemie II \\ Direktor/in: Prof. Dr. Ivan Dikic
}

\title{
Proteome dynamics upon proteotoxic stress and disease
}

\author{
Dissertation \\ zur Erlangung des Doktorgrades der theoretischen Medizin \\ des Fachbereichs Medizin \\ der Johann Wolfgang Goethe-Universität \\ Frankfurt am Main \\ vorgelegt von \\ Kevin Klann \\ Master of Science \\ aus Frankfurt am Main
}

Frankfurt am Main, 2020 
Dekan:

Referent/in:

Korreferent/in:

ggf. 2. Korreferent/in:

Tag der mündlichen Prüfung:
Prof. Dr. Stefan Zeuzem

Dr. Christian Münch

Prof. Dr. Thomas Oellerich

Prof. Dr. Urs Christen

20.04.2021 
This work is dedicated to Kristina and my whole beloved family. Thank you for your support on the way through these years. 


\section{Table of contents}

$\begin{array}{lll}1 & \text { Kurzzusammenfassung der vorgelegten Arbeit } & 7\end{array}$

2 Short summary of the presented work 9

3 Acronyms 11

4 Introduction 12

4.1 Protein dynamics . . . . . . . . . . . . . . . . . . . . . . 12

4.2 The effect of cellular stress on protein dynamics . . . . . . . . . 13

4.3 Protein dynamics during infection . . . . . . . . . . . . . . . . . . 14

4.4 Measurements of protein dynamics . . . . . . . . . . . . . . . . 16

5 Manuscripts 17

5.1 Multiplexed enhanced protein dynamics mass spectrometry . . . . 17

5.2 Protein dynamics upon infection with SARS-CoV-2 . . . . . . . . . 19

6 Discussion

6.1 Unbiased measurements of protein synthesis . . . . . . . . . . 21

6.2 Translational control upon stress . . . . . . . . . . . . . . . . 22

6.3 Implications for disease biology . . . . . . . . . . . . . . . . 23 
6.4 Dynamic protein changes upon viral infection . . . . . . . . . . . . 24

7 Published works $\quad 26$

7.1 Functional Translatome Proteomics Reveal Converging and DoseDependent Regulation by mTORC1 and elF2 $\ldots$. . . . . . . . . 26

7.2 Instrument Logic Increases Identifications during Multiplexed Translatome Measurements . . . . . . . . . . . . . . . . . . . 45

7.3 Unbiased translation proteomics upon cell stress . . . . . . . . . 51

7.4 Proteomics of SARS-CoV-2-infected host cells reveals therapy targets ........................ 55

7.5 Growth factor receptor signaling inhibition prevents SARS-CoV-2 replication . . . . . . . . . . . . . . . . . 62

8 Presentation of own work on manuscripts 89

8.1 Functional Translatome Proteomics Reveal Converging and DoseDependent Regulation by mTORC1 and elF2a, 2020, Molecular Cell 89

8.2 Instrument Logic Increases Identifications during Mutliplexed Translatome Measurements, 2020, Analytical Chemistry . . . . . . . . . 89

8.3 Unbiased translation proteomics upon cell stress, 2020, Molecular and Cellular Oncology . . . . . . . . . . . . . . . . . . . . . . . . . 89

8.4 Proteomics of SARS-CoV-2-infected host cells reveals therapy targets, 2020, Nature (Co-First author) . . . . . . . . . . . . . . . . 90 
8.5 Growth factor receptor signaling inhibition prevents SARS-CoV-2 replication, 2020, Molecular Cell (Co-First author) . . . . . . . . 90

9 References $\quad 91$

10 Acknowledgements

$\begin{array}{ll}11 \text { Curriculum Vitae } & 103\end{array}$

12 Schriftliche Erklärung 


\section{Kurzzusammenfassung der vorgelegten Arbeit}

Die Funktion aller Zellen wird durch ein komplexes Netzwerk von Proteinen aufrechterhalten, welches nach Bedarf neue Proteine synthetisiert und beschädigte, beziehungsweise nicht benötigte Enzyme/Proteine abbaut. Dieses Netzwerk der sogenannte Proteinhomeostase benötigt Regulation auf unterschiedlichen Ebenen um korrekt zu arbeiten. Störungen dieser Maschinerie sind an der Pathogenese von malignen Neoplasien und neurodegenerativen Erkrankungen beteiligt. Außerdem beeinträchtigen verschiedene humanpathogene Bakterien und Viren dieses Netzwerk der Proteinhomeostase. Um diese Krankheitsbilder besser zu verstehen ist es daher notwendig die Regulation dieses Netzwerks und die Auswirkungen von Pertubationen auf zellulärer Ebene zu beobachten um Rückschlüsse auf die Pathogenese ziehen zu können. Es konnten bereits für einige Krankheiten Therapieansätze entwickelt werden, die das ProteinhomeostaseNetzwerk gezielt modulieren ${ }^{1-3}$. Trotz dieser Fortschritte ist weiterhin für viele Störfaktoren unklar, wie sie das Proteinnetzwerk der Zelle beeinflussen. Dies ist teilweise dem Umstand geschuldet, dass die resultierenden Effekte innerhalb der Zelle hochdynamisch und daher oft schwer messbar sind. Teil dieser Arbeit war es eine Messmethode zu entwickeln, die es ermöglicht die Auswirkungen von proteotoxischem Stress, d.h. Störungen der Proteinhomeostase, auf die Dynamik von Proteinsynthese und -abbau in der Zelle mit Hilfe von Massenspektrometrie (MS) zu untersuchen. Um diese Messungen zu ermöglichen wurde eine neue Methode entwickelt, multiplexed enhanced protein dynamic (mePROD) MS, welche durch besondere Probenzusammenstellung und Datenanalyse ermöglicht, schnelle und transiente Veränderungen (im Zeitraum von Minuten) in der Proteinbiosynthese von tausenden Proteine zu messen ${ }^{4}$. Zwei der prominentesten Regulatoren der Proteinbiosynthese die Integrated Stress Response (ISR) und mammalian target of rapamycin (mTOR) werden mit vielen verschiedenen Krankheiten in Verbindung gebracht ${ }^{2,5}$ und sind unter anderem Ziele für neue Therapien in der Onkologie. Obwohl beide die Proteinbiosynthese beeinflussen, wurden sie bisher als Signalwege mit unterschiedlichen Effekten auf die Protein- 
synthese gesehen. Mittels der neu entwickelten mePROD-Methode konnte im Rahmen dieser Arbeit erstmals der globale Effekt beider Regulationswege auf das Proteinnetzwerk gezeigt werden. Es fanden sich überlappende Muster zwischen mTOR- und ISR-vermittelter Regulation der Proteinsynthese. Dies verändert die bisherige Modellvorstellung für diese zellulären Vorgänge und stellt eine wichtige Ressource für das Forschungsfeld dar ${ }^{4,6}$. Des Weiteren wurde die entwickelte mePROD Methodik verbessert um den Probendurchsatz zu erhöhen ${ }^{7}$. Durch die Kombination mit logik-basierter Messmethoden, konnte die Anzahl der durch mePROD quantifizierten Proteinen bei gleicher Messzeit verdreifacht werden.

Die Dynamik von Proteinsynthese und -abbau wird häufig während Infektionen verändert um die Vermehrung des Krankheitserreger zu ermöglichen oder selbiges zu bekämpfen. Für eine rationale Entwicklung von Therapien gegen diese Infektionen ist es daher unumgänglich die Veränderungen ausgehend von dem Erreger zu erfassen, zu verstehen und die Antwort der Wirtszelle auf die Infektion zu charakterisieren. Ende des Jahres 2019 wurde ein neues Virus entdeckt, SARS-CoV-2, welches sich, hochinfektiös, rasant verbreitet hat und zu einer weltweiten Pandemie geführt hat ${ }^{8-10}$. Um das neuartige Virus zu verstehen und auf Basis molekularbiologischer Erkenntnisse Therapieansätze zu entwickeln, haben wir die zuvor entwickelte mePROD Methode angewandt um die dynamischen Veränderungen im Wirtsproteom zu erforschen. Es konnte erstmals beobachtet werden, dass nach Infektion mit SARS-CoV-2 verschiedenste Prozesse dereguliert wurden (mRNA Splicing, Glykolyse, DNS Synthese oder Proteinhomeostase $)^{11}$. Durch gezielte Inhibierung dieser Prozesse konnte gezeigt werden, dass diese Funktionen der Wirtszelle wichtig für die Replikation von SARS-CoV-2 und durch Medikation modulierbar sind. In einer weiteren Studie konnte zudem durch Analyse von zellulären Signaltransduktionswegen nachgewiesen werden, dass die Aktivierung von Wachstumsfaktor-Signalwegen für die effiziente Replikation der Viren notwendig ist ${ }^{12}$. Die getestete Anwendung von Medikamenten, die bereits zugelassen (Ribavirin, Sorafenib) oder sich in klinischen Studien befinden, blockierte die Virusreplikation vollständig in vitro und zeigt somit mögliche, in die Klinik übertragbare, Therapieansätze. 


\section{Short summary of the presented work}

Correct cellular function is ensured by a complex network of proteins and enzymes, regulating protein synthesis and degradation. This protein network, maintaining the so-called protein homeostasis, regulates those processes on multiple levels, producing new or degrading old proteins to cope with changing intra- and extracellular environments. Disturbance of this tightly regulated machinery can have severe effects on the cell and can lead to a variety of pathologies on organism level. Diseases including cancer, neurodegeneration and infections are associated with causative or consequent alterations in protein homeostasis. To understand the pathologies of these diseases, it is therefore critical to examine how perturbations of protein homeostasis affect cellular pathways and physiology. In the recent years, analysis of protein homeostasis networks has resulted in the development of novel therapeutic approaches ${ }^{1-3}$. However, for many factors it remains unclear how the cell is affected, if they are disturbed. Protein synthesis and degradation represent immediate responses of the cell to changes and need to be studied in the right timeframe, making them difficult to access by common methodology. In this work we developed a new mass spectrometry (MS) based method to study protein synthesis and degradation on a system-wide scale. Multiplexed enhanced protein dynamic (mePROD) MS was developed, overcoming these limitations by special sample mixing and novel data analysis protocols. MePROD thereby enables the measurement of rapid and transient (e.g. minutes) changes in protein synthesis of thousands of proteins ${ }^{4}$. During responses of the cell to stressors (e.g. protein misfolding, oxidation or infection), two major pathways regulate the protein synthesis: the Integrated Stress Response (ISR) and mammalian target of rapamycin (mTOR). Both pathways have been connected with various diseases in the past ${ }^{2,5}$ and are common therapy targets. Although both pathways target protein synthesis in stress responses, the set of targets regulated by these pathways was believed to differ. Through the new mePROD MS method we could measure a comprehensive comparison of both pathways for the first time, revealing comparable system-wide patterns of regulation between 
the two pathways. This changed the current view on the regulation elicited by these pathways and furthermore represents a useful resource for the whole field of research. We could further develop the mePROD method and decrease MS measurement time needed to obtain an in-depth dataset ${ }^{7}$. Through implementation of logic based instrument methods, it was possible to enhance the number of measured proteins by approximately three-fold within the same measurement time.

The dynamics of protein synthesis and degradation are frequently modulated by pathogens infecting the cell to promote pathogen replication. At the same time, the cell counteracts the infection by modulating protein dynamics as well. To develop useful therapy approaches to fight infections, it therefore is necessary to understand the complex changes within the host cell during infections on a system-wide scale. In 2019, a novel coronavirus spread around the world, causing a world-wide health-crisis ${ }^{8-10}$. To better understand this novel virus and its infection of the host cell we conducted a study applying the mePROD methodology and classical proteomics to characterize the dynamic changes during the infection course in vitro. We discovered that the infection remodeled a diverse set of host cell pathways (e.g. mRNA splicing, glycolysis, DNA synthesis and protein homeostasis) and thereby showed possible targets for antiviral therapy ${ }^{11}$. By targeted inhibition of these pathways, we could observe that these pathways indeed are necessary for SARS-CoV-2 replication and their inhibition could reduce viral load in the cells. Another experimental approach focused on the dynamic changes of protein modification, namely phosphorylation, after infection with SARS-CoV-2 ${ }^{12}$. Here, we could show the very important participation of growth factor signaling pathways in viral proliferation. Both studies together revealed critical pathways that are needed for the viral proliferation and hence are promising candidates for further therapies. Subsequent targeting of these pathways by either already approved drugs (Ribavirin and Sorafenib) or drugs in clinical trials (2-deoxyglucose, PladienolideB, NMS-873, Pictilisib, Omipalisib, RO5126766 and Lonafarnib) could block viral replication in vitro and suggests important clinical approaches targeting SARSCOV-2 infection. 


\section{Acronyms}

$\begin{array}{cc}\text { AHA } & \text { Azido-homoalanine (methionine analogue) } \\ \text { ATP } & \text { Adenosine triphosphate } \\ \text { DDA } & \text { Data dependent acquisition } \\ \text { GFR } & \text { Growth factor receptor } \\ \text { ISR } & \text { Integrated stress response } \\ \text { mePROD } & \text { Multiplexed enhanced protein dynamics } \\ \text { MS } & \text { Mass spectrometry } \\ \text { mTORC1 } & \text { mTOR complex I } \\ \text { mTOR } & \text { Mammalian target of rapamycin } \\ \text { NGS } & \text { Next generation sequencing } \\ \text { SILAC } & \text { Stable isotope labeling in cell culture } \\ \text { TMD } & \text { Targeted mass difference } \\ \text { TMT } & \text { Tandem mass tags } \\ \text { UPR } & \text { Mitochondrial unfolded protein response }\end{array}$




\section{Introduction}

\section{Protein dynamics}

The central dogma of cell biology relies on the protein synthesis from mRNA transcribed complementary to the genomic DNA. While it is nowadays easily possible to predict all possible protein sequences derived from the genome, the synthesis event itself is highly regulated and not every protein is expressed in every tissue and condition. Furthermore, in recent years, studies showed that the level of total transcribed mRNA often does not correlate with the resulting protein levels in the cell $^{13}$. This phenomenon is the result of multiple layers of regulation at the protein level, starting with synthesis, followed by, often transient, modifications and finally, ending with degradation (Fig. 1). Each layer on its own has an influence on the final protein amount and function.

The synthesis machinery of proteins is controlled by a plethora of events managing the translational status of an $\mathrm{mRNA}^{14}$. Regulatory networks integrate a variety of signals (such as metabolic states, extracellular changes or stressors) and differentially control mRNA translation accordingly. Subsequently, the produced polypeptides emerging from the ribosome can be heavily modified by enzymes or chemicals. These modifications can be highly dynamic and often regulate protein function ${ }^{15}$. While some modifications (e.g. some oxidation states) are irreversible, others, such as phosphorylation, are not. Modification of proteins and enzymes thereby represents a fast molecular switch for their functions, without the energy needed to synthesize or degrade the protein. The last major component of the proteome dynamic regulatory network is the degradation machinery. Proteins are constantly turned over, either due to irreversible modification, damage or functional reasons, by several systems working in conjunction to control protein levels $^{16,17}$. The degradative system as well is highly dynamic and constantly adapts to the cellular needs.

The dynamic equilibrium of these processes has been termed "protein home- 
ostasis" (or "proteostasis"). Disturbance of protein homeostasis and subsequent deregulation of protein dynamics can be both cause and/or effect of various diseases, such as cancer, infection or neurodegeneration ${ }^{2,5,18-20}$, thus, having high pathological relevance. During these pathologies, protein dynamics are subject to several regulatory events. On one hand, diseases can cause the proteostasis to collapse, altering the properties and composition of the proteome. On the other hand, cells have innate sensors for irregular protein homeostasis, triggering response mechanisms trying to restore proteome balance. Both aspects happen at the same time, making quantitative assessment of the resulting changes mandatory. To therapeutically tip this balance in favor of the patient, it is therefore critical in the first step to measure and understand the proteome changes happening during human pathologies, to derive potential candidates to target by therapies.
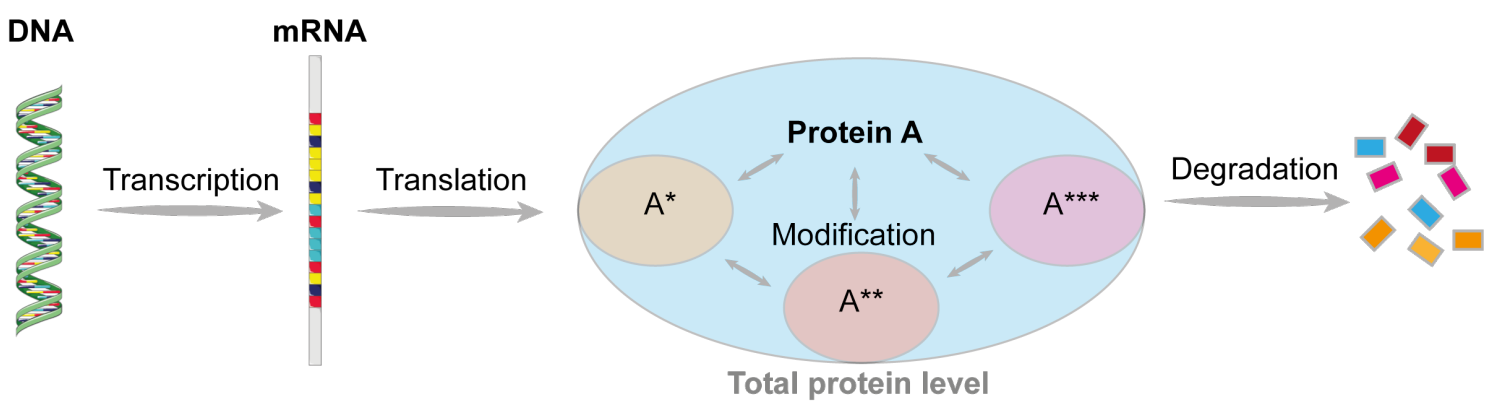

Figure 1: Scheme of the central dogma of cell biology. Gene information is transcribed into mRNA, which in turn is translated into the protein. The pool of the resulting protein is partially modified and degraded, reaching a dynamic equilibrium of protein synthesis, modification and degradation.

\section{The effect of cellular stress on protein dynamics}

As stated before, the regulatory networks controlling proteostasis sense and integrate diverse cellular stress signals. Stress in this context can range from metabolic stress, such as adenosine triphosphate (ATP) depletion, over viral or bacterial infections to proteotoxic stress elicited by damage to proteins/enzymes or mutations. These stresses are recognized by various sensors in the different cellular compartments and signaled to downstream targets. The signaling events from 
stress are diverse and have different results, depending on cell type and stressor. For example, some cascades can result in inflammatory responses, elevating expression levels of cytokines, while some others can increase the capacity of chaperones to increase cellular maintenance capacity. While some response types are specific for certain stressors (e.g. antioxidant genes during oxidative stress; strong immune reponse during infection), some pathways are common between most stressors. These common pathways are signaling hubs inside the cell that integrate information from different potential stress sources (Fig. 2). A prominent and important example is the translational control after sensing cellular stress. There are two major pathways controlling protein translation upon stress on multiple levels: 1) the integrated stress response (ISR), which acts by dynamic phosphorylation of the eukaryotic translation initiation factor 2 alpha (elF2 $\alpha$ or EIF2S1), thereby limiting its activity ${ }^{21-23}$ and, 2) the mammalian target of rapamycin complex 1 (mTORC1), which regulates protein synthesis by phosphorylation of ribosomal protein S6 kinase 1 (S6K1) and the eukaryotic initiation factor 4E binding protein 1 (EIF4EBP1) ${ }^{24,25}$. Both pathways have been extensively studied for their translational regulation ${ }^{26-28}$, mainly by next generation sequencing (NGS) based methods, and have been viewed as substantially different in context of their effect on translation. Notably, both pathways not only directly regulate protein synthesis, but also elicit transcriptional and degradative changes, shaping the stress response proteome.

\section{Protein dynamics during infection}

Both ISR and mTOR pathways and the corresponding changes in protein dynamics have not only found associated with cancer or neurodegeneration, but also been linked to various infectious diseases. Both bacterial and viral infections are known to modulate protein synthesis, modification and degradation, resulting in diverse effects on the host cell, which are not yet fully understood. Bacteria, such as Legionella sp. or Salmonella sp., can secrete effector proteins into the host cell that modulate cellular translation via mTORC1 or reshape the ubiquitin system to change autophagy and degradation ${ }^{29-31}$. While bacteria usually secrete 


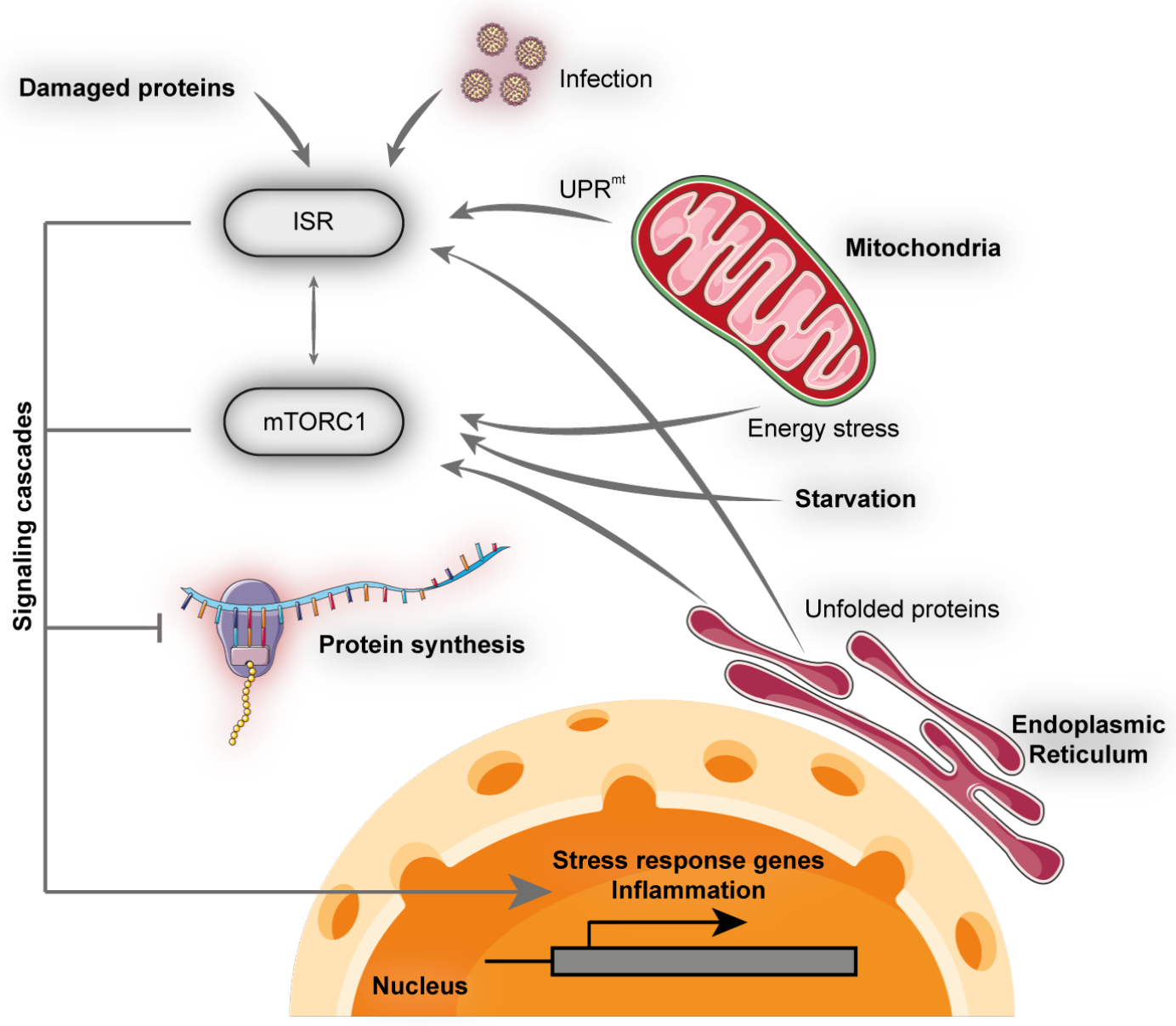

Figure 2: Simplified scheme of cellular stress responses. The integrated stress response (ISR) and mammalian target of rapamycin complex 1 (mTORC1) integrate stress signals from various sources (e.g. unfolded/damaged proteins in different compartments [cytosol, endoplasmic reticulum or mitochondria; UPR ${ }^{m t}$ ], energy stress, starvation or infections) and trigger multiple signaling cascades. Both pathways inhibit protein synthesis and drive expression of different sets of stress response genes (e.g. inflammatory genes such as cytokines, chaperones or antioxidant responses).

proteins to interact with the host proteome, viruses release their genome into the host to hijack machineries for virus production. Both RNA and DNA viruses are known to manipulate the host translation machinery to efficiently translate viral proteins $^{3,20,32-34}$.

In case of RNA viruses, protein synthesis is not only regulated by the synthesized 
viral proteins, but is in turn also commonly repressed by the host cell via the ISR as a countermeasure ${ }^{35,36}$. The viral RNA genome is sensed by the double-stranded RNA activated kinase protein kinase $R(P K R)$, which is one of the four kinases driving the $\mathrm{ISR}^{23}$, thus leading to translational modulation and important antiviral signaling. However, many viruses are able to evade such antiviral responses by a variety of different mechanisms ${ }^{34,37}$. Direct interaction with the cellular translation components can force production of viral genes, albeit antiviral signaling ${ }^{34,38,39}$. In addition, the directed degradation of important host molecules can lead to antagonistic effects on inflammatory responses and antiviral signaling ${ }^{40,41}$.

Notably, pathogens not only affect protein synthesis and degradation, but they also dynamically modulate protein function either directly ${ }^{42}$ or indirectly by changes in the modification states ${ }^{43}$. Hence, during infections the host proteome is reshaped on multiple levels, creating a tug-of-war between pathogen and host over the host cellular machineries. Assessment of these changes has proven important to understand the disease and reveal treatment options ${ }^{43,44}$.

\section{Measurements of protein dynamics}

In the recent years mass spectrometry has taken the lead in proteomics and currently represents the state of the art technology to measure protein levels ${ }^{45}$. With more sensitive machines and sophisticated workflows, quantitative measurements of levels from $>12,000$ proteins $^{46}$ are possible together with deep profiling of modification sites, such as phosphorylation ${ }^{47,48}$. However, the quantitative assessment of protein synthesis and degradation has proven more difficult and fewer advances have been made so far. Protein dynamics are commonly measured by incorporation of labeling agents, such as isotope marked amino acids (stable isotope labeling in cell culture; SILAC ${ }^{49}$ ), unnatural amino acids (e.g. Azidohomoalanine; $\mathrm{AHA}^{50}$ ) or puromycin ${ }^{51}$. These labeling reagents enable to distinguish the pre-existing proteins from newly synthesized proteins during the labeling time by a mass shift. Due to the higher complexity of the sample, the depth of such measurements is intrinsically reduced. Additionally, while studying rapid changes in protein synthesis/degradation the signal of interest is usually of very low inten- 
sity and thus measurement is restrained by technical limitations. To overcome these limitations several approaches have been developed by either multiplexing samples by Tandem Mass Tags (TMT) ${ }^{52}$ or enrichment of labeled moieties by affinity purification ${ }^{50,51}$. TMT reagents enable the pooling of up to 16 samples in one measurement by modifying peptides with different isobaric reporter reagents. After labeling, the modified peptides from different samples can be pooled and measured simultaneously. The labeling reagents will modify peptides from all samples with the same mass, thereby adding no additional complexity in the survey scan. Upon fragmentation (that is commonly done to identify the peptide sequence) the labeling reagent will fall apart into (up to 16) different reporter ions, depending on the sample. This then allows accurate quantification of the multiplexed samples within the same MS run, strongly reducing technical variation. Nevertheless, for measurements of protein dynamics these approaches still lack either the detection of rapid changes due to long labeling times (pulsed SILAC / $\mathrm{TMT}^{52}$ ) or reasonable depth $^{50,51}$. Additionally, the incorporation of unnatural amino acids intrinsically disturbs the observed system, due to differences in biology ${ }^{6,53}$, rendering them prone to experimental artifacts.

\section{Manuscripts}

\section{Multiplexed enhanced protein dynamics mass spectrometry}

In the manuscript "Functional Translatome Proteomics Reveal Converging and Dose-dependent Regulation by mTORC1 and elF2 $\alpha$ ", a novel advancement in protein dynamic profiling has been established ${ }^{4}$. Pulsed SILAC combined with TMT multiplexing and subsequent addition of a "boost" channel, amplified the signal of interest, which can now be measured more reliably by the MS-instrument. This, in conjunction with a custom-built data analysis algorithm, significantly enhanced the identification rates for newly synthesized proteins, while allowing short labeling times (time-scale of minutes) to study rapid changes within the cell. Application of this novel method to study the direct effects of cellular stress on protein 
synthesis regulation revealed that the two major pathways responsible for stress integration to translation (ISR and mTORC1) have a largely overlapping set of translationally regulated targets. Contrasting the commonly held view picture of translation regulation upon stress, our results showed an intrinsic tendency of each mRNA for translational repression upon stress, independent of the underlying pathway, that takes place in a gradual manner. Protein synthesis is predominantly fine tuned on a gradual and continuous scale rather than being strictly binary and switching between an 'on' and 'off' state in response to cellular needs. Since both pathways are frequent target of therapies and implicated in a variety of diseases ${ }^{2,18,54,55}$, these findings shed new light on the effect of such therapies and might implicate novel approaches to modulate protein synthesis. Besides the biological insights gained through this study, the methodology presents a major advancement in the field of protein dynamics. The mePROD method allows short labeling times to reach high temporal resolution, while allowing in-depth analysis, in an unbiased system. Previous approaches, as described above, used the incorporation of non-canonical substances, thereby disturbing the biological system studied. This issue is also discussed in our authors view manuscript - "Unbiased

\section{translation proteomics upon cell stress"6.}

Albeit reaching sophisticated depth of analysis during mePROD measurements, extensive pre-fractionation and measurement time was needed to perform these experiments. Although boosted by the carrier channel, the signal of interest, compared to the rest of the sample, still only represents $10 \%$ of the intensity range. Hence, during classical measurements (data dependent acquisition; DDA) on MS instruments, most of the measurement time is used to quantify the pre-existing proteome and rather than the newly synthesized fraction. Mass spectrometers usually select precursors ranked by their intensity, intrinsically biasing the data towards the highly abundant background proteome. To enable higher identifications, while reducing the sample input required, an alternative approach to DDA was introduced and benchmarked in our follow up study "Instrument Logic Increases Identifications during Mutliplexed Translatome Measurements"7. Here we introduced an alternative instrument set up to identify the introduced label directly during the measurement together with its unlabeled counterpart, subject- 
ing both to quantification. This selection is mostly independent on the intensity of the labeled moiety, overcoming previous limitations. Thereby, the bias towards the background proteome is reduced, since for each high-abundant background peptide, the low-abundant counterpart is also quantified. In addition, the measurements of both (labeled and unlabeled) partner peptides enables robust normalization, solidifying the resulting quantification. The more efficient use of machine time, hence, enables higher sample throughput, since less time is needed per sample, makes the method suitable for low input samples (e.g. patient samples or primary cells) and, extensive pre-fractionation can be avoided.

\section{Protein dynamics upon infection with SARS-CoV-2}

The proteome of host cells during infection is subject to highly dynamic changes on many layers. Hence, measuring protein dynamics during an infection can provide with critical data to understand the course of disease and suggest treatment options. In 2019, a novel and highly infectious coronavirus, SARS-CoV2 , emerged causing a worldwide health crisis. Although exhibiting similarities to other coronaviruses, such as SARS-CoV ${ }^{56}$, pathophysiological differences could be observed ${ }^{57}$. Besides extensive screening for drugs, rational selection of candidate drugs based on cell-biological insights is needed to rapidly suggest treatment options. In the study "Proteomics of SARS-CoV-2-infected host cells reveals therapy targets"11, the previously developed mePROD method was applied, in conjunction with classical quantitative proteomics, to a cell line model infected with SARS-CoV-2 intact virus, previously isolated in Frankfurt ${ }^{58}$. The study showed that the used Caco-2 cell line was highly permissive for SARS-CoV-2 and represents a valid model system to study the viral infection. Furthermore, protein dynamic profiling revealed, that in contrast to other RNA viruses ${ }^{3,20,32}$, SARS-CoV-2 exhibited only minor effects on protein synthesis, which likely is the effect of strong increase in the host translational machinery as measured by mePROD. By measuring the proteome's course during infection, it was observed that several additional pathways were significantly altered (splicing, glycolysis, proteostasis and DNA synthesis), suggesting important changes during infection. Indeed, targeting 
these pathways by repurposing drugs previously used in clinical tests (ementine, pladienolide-B, 2-deoxy-glucose, ribavirin and NMS-873) ${ }^{59-64}$ showed significant repression of viral replication at clinically achievable concentrations. The results of this in vitro proteomics dataset offers promising potential treatment options.

Previous studies on other viruses revealed strong changes in the dynamics of protein phosphorylation during infection ${ }^{43,44}$. However, comprehensive data for SARS-CoV-2 was not available. Hence, we performed deep phospho-proteomic profiling combined with quantitative proteomics to study system-wide signaling network dynamics upon infection"12. The study "Growth factor receptor signaling inhibition prevents SARS-CoV-2 replication" shows extensive reprogramming of host cell protein modification and signaling. Bioinformatic drug-target network analysis of the most significantly changed signal transduction pathways revealed a central role of the growth factor receptor (GFR) signaling cascade in viral proliferation. Downstream inhibition of two major axes (phospho-inositol 3 kinase ; PI3K and mitogen activated protein kinase ; MAPK) of GFR signaling by repurposed drugs (pictilisib, omipalisib, sorafenib, lonafarnib and R05126766) ${ }^{65-68}$ could efficiently block SARS-CoV-2 replication. These results significantly added to the understanding of the new disease and opened new treatment options that now need to be validated in clinical settings.

Taken together, a new major advancement for the field of protein dynamics mass spectrometry was established, used to dissect fundamental cell biological processes under stress conditions and transferred to a highly relevant disease model. Profiling proteome dynamics on multiple levels successfully revealed treatment options for a novel disease. In combination with quantitative modification profiling and whole cell proteomics, system-wide proteome measurements could discover critical pathways during SARS-CoV-2 infection. Modulation of these pathways by available drugs lead to a complete block of viral proliferation at clinically achievable concentrations and is currently independently evaluated ${ }^{69,70}$ in the clinic. 


\section{Discussion}

\section{Unbiased measurements of protein synthesis}

While the measurement of total protein levels by proteomics improved a lot during the last years, the assessment of protein dynamics has intrinsic challenges - 1) During protein synthesis measurements the signal of interest usually has a very low intensity, making reproducible detection difficult. Enrichment of this signal by affinity purification has been established in the past ${ }^{71}$ and widely used. However, the necessary use of non-canonical amino acids intrinsically influences the observed system by altering protein synthesis kinetics ${ }^{6,53} .2$ ) Non-biased approaches using SILAC require long labeling times to reach sufficient signal intensity. Thus, no good temporal resolution can be achieved. 3) NGS based methods have an inherent bias during sample preparation and data analysis, rendering them unable to detect global changes ${ }^{72,73}$. Although they exhibit high measurement depth, quantification is biased. mePROD profiling largely overcomes these limitations, by combining SILAC based labeling with TMT multiplexing and a unique sample combination strategy $y^{4,7}$. The used amino acids enable labeling without interfering with the studied system, since they harbour naturally occurring isotopes. The combination of samples with a boost channel greatly increases the signal intensity of labeled peptides, thereby increasing the identification and quantification rate without affecting quantification quality. Increasing the signal intensity also greatly enhances temporal resolution, making labeling times of minutes possible without the need for enrichment. Through the simultaneous measurement of already existing and newly synthesized proteins, quantification bias is eliminated, making global changes accurately accessible. We further broadened this approach applying machine logic filters to the measurement method ${ }^{7}$. Deep translatome measurements by the original mePROD protocol originally required a large amount of sample material, easily obtainable in cell culture, but difficult to obtain when working with primary cells or patient material. By efficient use of acquisition time on the mass spectrometer, 1) the previously required fractionation depth was reduced, decreasing the input material required, making it more applicable 
to limited (patient) material, and 2) the total measurement time on the instrument is reduced, which limits costs and enables higher throughput of samples.

\section{Translational control upon stress}

The power of the developed method was successfully demonstrated by the application to various scenarios. We showed that mePROD enables faithful quantification of cellular translation upon induction of stress response pathways ${ }^{4}$. This unbiased approach shed new light on translation regulation upon stress. mRNA translation was believed to be targeted specifically by stress response pathways, such as the ISR or mTOR. Through our comprehensive datasets, we showed that, in contrast to the common view, the majority of mRNAs is translationally regulated independent of the upstream pathway. The bulk pool of mRNAs exhibits a dosedependent translation attenuation that is dependent on the global levels of protein synthesis within the cell. While the majority of mRNAs follows global translation trends, subsets of mRNAs seem intrinsically resistant (or especially sensitive) to repression of the translation machinery. The nature of this resistence (or sensitivity, respectively) remains unclear and will require further studies. Possible mechanisms include RNA structural elements, which are a common strategy of viral mRNAs to modulate their efficient translation ${ }^{74}$. Interaction with the translating or initiating ribosomes could drive differential sensitivity to translational arrest by shaping the accessibility of the mRNA to the limited ribosome pool. Another hypothesis would be the participation of RNA binding proteins, that modulate translation efficiency. In the past years newly developed methods identified hundreds of previously unknown proteins with the ability to bind mRNA ${ }^{75}$. These proteins likely control translational, as has been shown for some single cases $^{76}$. Differential control of protein synthesis on the level of the ribosome expands the list of possible regulatory events. Specialized ribosomes, exhibiting alternative compositions and/or modifications, have been described ${ }^{77}$, but so far our understanding of these mechanisms is limited. The mechanisms of translation regulation will most likely include a number yet unknown factors and functions. Understanding such mechanisms in detail, will change the understanding of proteostasis-related 
diseases (e.g. neurodegeneration) and could lead to the discovery of new therapeutics.

\section{Implications for disease biology}

Deregulation of translation control is a hallmark of many diseases. Aberrant mTOR signaling is often found in various cancers. Common mutations in the pathway include activation of the PI3K/AKT axis (e.g. PIK3CA H1047 or AKT $\mathrm{E} 17 \mathrm{~K})$, inactivation of the TSC1/TSC2 complex or activating mutants of mTOR itself $^{78}$. Through promoting translation, activated mTOR drives cancer proliferation. Hence, it has been selected as a therapeutic target and various inhibitors (mainly rapamycin analogues; Sirolimus, Everolimus or Temsirolimus) are in clinical use. However, the success of these treatments for cancer is limited, since often feedback loops confer resistance to the treatment ${ }^{79-81}$. Understanding the translational outcome of sustained mTOR inhibition could be critical for the knowledge of resistance formation. Diseases linked with the ISR paint a similar picture. Mutations in ISR components mostly affect brain and pancreas and are linked with a plethora of cognitive disorders and neurological symptoms ${ }^{82}$. These mutations often target the eukaryotic initiation complex elF2 and thereby have direct effects on translation control. Although the molecular mechanism how these mutations affect translation (limitation of the ternary initiation complex) are known, the result for cells and tissue is not clear. Translatome studies employing mePROD to dissect the translation and proteome landscape in a disease reflecting mutational background might shed new light on these pathologies. Especially in the light of recent studies, where modulation of the ISR with the small molecule $\mathrm{ISRIB}^{27,83}$, blocking the translation repression, shows in vitro efficiency against the MEHMO syndrome ${ }^{84}$, in vivo effects in a down syndrome mouse model ${ }^{85}$, and other disease models ${ }^{2,86}$. These results additionally highlight the importance of our understanding of translation control for many diseases. 


\section{Dynamic protein changes upon viral infection}

Studying dynamic protein networks can be useful to unravel infection related pathways, for direct medical intervention. Application of multiple proteomic approaches led to the discovery of important pathways in SARS-CoV-2 infection that are targetable by small molecule compounds. We observed significant changes in the expression of spliceosome components and several metabolic pathways (e.g. glycolysis, nucleotide biosynthesis). Interestingly, many of the identified components of the splicing machinery have been found to be associated with other viruses previously ${ }^{87-89}$. Indeed, targeting the spliceosome with pladienolide $B^{90}$ showed impaired viral replication within the host cell. Similar effects we observed with the metabolic pathways found, where inhibition of the glycolytic enzymes by 2-DG and interference with the nucleotide biosynthesis by ribavirin showed antiviral effects in our system. Notably, ribavirin is already approved for therapy and used to treat other viral infections (hepatitis $\mathrm{C}^{61}$ and $R S V^{60}$ ), enabling rapid repurposing studies to treat COVID-19.

Protein modification are commonly altered during infection, changing signaling cascades and/or protein functions. While some important pathways exhibit changes directly on protein levels (i.e. synthesis or total protein pool), others can only be identified while profiling protein modification states. We performed global phosphorylation analysis of cells infected with SARS-CoV-2 and, by comprehensive network analysis we identified additional pathways important for viral replication. Our analysis showed growth factor signaling downstream signaling to play a central role during SARS-CoV-2 infection. These results are consistent with other studies, showing a clear connection between GFRs and diverse viral infections ${ }^{91-95}$. GFRs and their downstream effectors have been extensively studied for cancer therapeutics ${ }^{96,97}$ and a variety of drugs have been developed ${ }^{66,98,99}$. We clearly showed that interference with these signaling axes, by repurposing available drugs blocked SARS-CoV-2 replication in vitro.

Taken together, our work shows that the examination of global protein dynamics (i.e. translation, dynamic protein level changes and modification) can improve 
understanding of diseases and lead directly to the discovery of druggable pathways. The quantification of translation, total protein levels and protein modification upon infection, combined with sophisticated bioinformatic network analysis revealed several key pathways needed for viral replication. Transferring the candidates to antiviral assays, in which we treated with already available drugs (such as ribavirin or sorafenib), showed significant reduction of viral load inside cells in vitro. This provides with the first step from bench-to-bedside. These drugs are now independently tested for their clinical efficiency for fighting the infection ${ }^{69,70 .}$ Analysis of protein network changes can reveal phenotypes and effects not visible on the level of single genes/proteins. Thus, protein dynamic measurements have broad future applications for medical and cell biological research, spanning nearly all disease relevant fields. 


\section{Published works}

Functional Translatome Proteomics Reveal Converging and DoseDependent Regulation by mTORC1 and eIF2a 


\section{Molecular Cell}

\section{Functional Translatome Proteomics Reveal Converging and Dose-Dependent Regulation by mTORC1 and elF2 $\alpha$}

Graphical Abstract

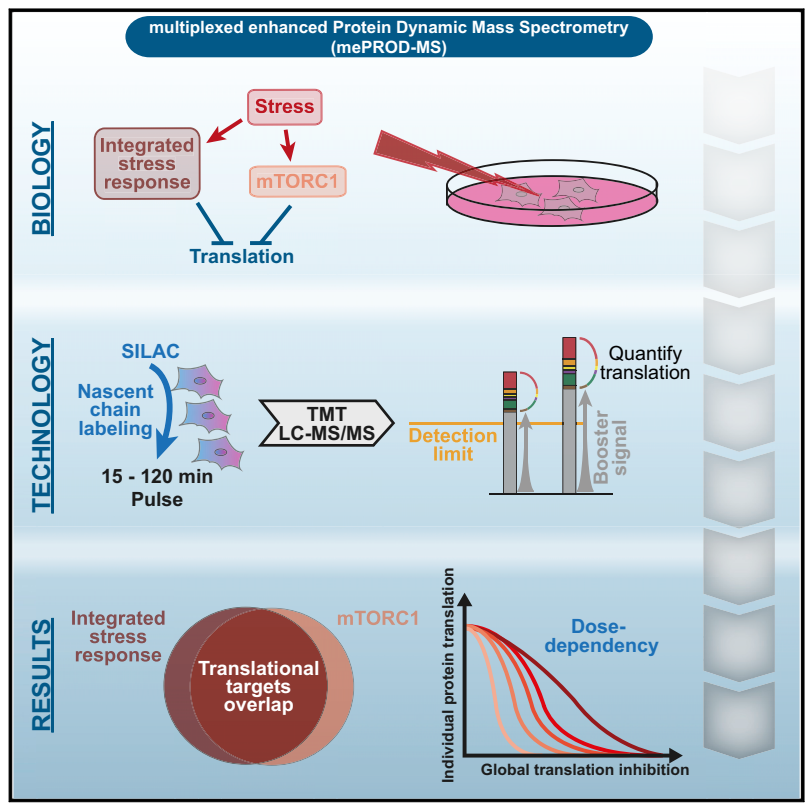

Highlights

- A proteomics method quantifies nascent protein chains minutes after labeling

- The integrated stress response and mTORC1 share translation targets

- Individual protein translation levels are driven by global translation status

\section{Authors}

Kevin Klann, Georg Tascher, Christian Münch

\section{Correspondence}

ch.muench@em.uni-frankfurt.de

\section{In Brief}

Klann et al. developed a proteomics method that uses signal amplification to enable acute translation measurements. Employing this method, they reveal that the integrated stress response and mTORC1 inhibit translation of the same set of proteins. Target specificity is defined by global translation attenuation in a dose-dependent manner. 


\section{Functional Translatome Proteomics Reveal Converging and Dose-Dependent Regulation by MTORC1 and elF2 $\alpha$}

Kevin Klann, ${ }^{1}$ Georg Tascher, ${ }^{1}$ and Christian Münch ${ }^{1,2,3,4, *}$

1 Institute of Biochemistry II, Faculty of Medicine, Goethe University, Frankfurt am Main, Germany

${ }^{2}$ Frankfurt Cancer Institute, Frankfurt am Main, Germany

${ }^{3}$ Cardio-Pulmonary Institute, Frankfurt am Main, Germany

${ }^{4}$ Lead Contact

*Correspondence: ch.muench@em.uni-frankfurt.de

https://doi.org/10.1016/j.molcel.2019.11.010

\section{SUMMARY}

Regulation of translation is essential during stress. However, the precise sets of proteins regulated by the key translational stress responses-the integrated stress response (ISR) and mTORC1-remain elusive. We developed multiplexed enhanced protein dynamics (mePROD) proteomics, adding signal amplification to dynamic-SILAC and multiplexing, to enable measuring acute changes in protein synthesis. Treating cells with ISR/mTORC1-modulating stressors, we showed extensive translatome modulation with $\sim 20 \%$ of proteins synthesized at highly reduced rates. Comparing translation-deficient sub-proteomes revealed an extensive overlap demonstrating that target specificity is achieved on protein level and not by pathway activation. Titrating cap-dependent translation inhibition confirmed that synthesis of individual proteins is controlled by intrinsic properties responding to global translation attenuation. This study reports a highly sensitive method to measure relative translation at the nascent chain level and provides insight into how the ISR and mTORC1, two key cellular pathways, regulate the translatome to guide cellular survival upon stress.

\section{INTRODUCTION}

Stress response mechanisms control cellular fate through multilayered regulation. Attenuation of translation is a rapid cellular response triggered by various stresses, such as the induction of the integrated stress response (ISR) and mTOR inhibition (Sonenberg and Hinnebusch, 2009). The ISR is driven by the phosphorylation of eukaryotic initiation factor 2 subunit 1 (elF2 $\alpha /$ EIF2S1) by one of four elF2 $\alpha$ kinases (EIF2AK1-4) and activated by diverse stresses, such as heme depletion (EIF2AK1/HRI), viral infection (EIF2AK2/PKR), ER stress (EIF2K3/ PERK), or amino acid deprivation (EIF2AK4/GCN2) (Taniuch et al., 2016). Phosphorylation of elF2 $\alpha$ causes tightened bind- ing to guanine nucleotide exchange factor elF2B, preventing formation of the $40 S$ preinitiation complex and leading to cellular translation attenuation (Kozak, 1999; Krishnamoorthy et al., 2001). Control of cellular translation by the ISR plays a central role in various diseases, such as diabetes, cancer, and viral infection (Back et al., 2009; Clavarino et al., 2012; Pakos-Zebrucka et al., 2016).

The mammalian target of rapamycin complex 1 (mTORC1) is the second major pathway mediating translational control in cells. Under basal conditions, mTORC1 phosphorylates the EIF4E binding proteins EIF4EBP1-3 and ribosomal protein S6 kinase (p70S6K1) (Sonenberg and Hinnebusch, 2009). EIF4EBP phosphorylation leads to dissociation from elF4E, enabling binding to elF4G and the formation of the initiation complex at the $5^{\prime}$-cap of mRNAs. Phosphorylation of p70S6K1 activates its kinase function and regulates translation by targeting EEF2K, EIF4B, and ribosomal protein S6 ( Holz et al., 2005; Raught et al., 2004; Wang et al., 2001). In response to low nutrient concentrations, mTORC1 becomes inactivated, resulting in hypo-phosphorylated EIF4EBP that subsequently binds elF4E and represses cap-dependent translation. Consequently, mTORC1 has major control over cellular behavior, and its regulation is modulated in numerous cancers (Sabatini, 2006). Studies monitoring EIF4EBP- and elF4E-dependent translation regulation identified a small subset of mRNAs to be controlled via this route (De Benedetti and Graff, 2004; Colina et al., 2008; Dowling et al., 2010; Graff and Zimmer, 2003; Roux and Topisirovic, 2012).

Despite both pathways regulating (albeit different) processes in translation initiation, elF2 $\alpha$ and $\mathrm{mTORC} 1$ are generally viewed as separate, translation-controlling pathways with specific outcomes (Wengrod and Gardner, 2015). A major focus of study of these translation-regulating pathways has been the analysis of downstream effects. Global analyses identifying and quantifying the specific translational output of translation regulation by elF2 $\alpha$ and mTOR have largely been carried out by ribosome profiling (Hsieh et al., 2012; Jiang et al., 2017; Reid et al., 2014; Sidrauski et al., 2015; Thoreen et al., 2012). These studies revealed a low number of differentially translated transcripts, despite showing extensive global downregulation of translation. This is largely due to a normalization procedure bias (Chen et al. 2015; McGlincy and Ingolia, 2017) that redistributes translation 
values back to unchanged global relative translation rates. As a result, transcripts do not reach sufficient statistical significance and/or fold changes (FC) to be identified as downregulated during translation attenuation by elF2 $\alpha$ or mTORC1 (Masvidal et al., 2017). Until today, conclusive datasets representing the set of proteins with reduced translation following elF2 $\alpha$ - or mTORC1-driven translation attenuation are not available. Thus, it remains unclear which proteins are translationally regulated by elF2 $\alpha$ and mTORC1 and whether these sets are indeed distinct and may be discriminated by additional features besides being capped.

In the last years, mass spectrometry (MS) approaches have helped in assessing protein dynamics by detecting protein degradation and synthesis, employing pulse-labeling of nascent peptide chains with heavy amino acid isotopes (SILAC) or clickreactive amino acids/puromycin (Becher et al., 2018; Jovanovic et al., 2015; Mathieson et al., 2018; Savitski et al., 2018; Schwanhäusser et al., 2009; Welle et al., 2016). A major limiting factor in the use of pulse-labeling newly synthesized proteins is the low stoichiometry of labeled proteins, preventing accurate, precise, and in-depth quantification (Münch and Harper, 2016). For basal protein degradation and synthesis experiments that monitor proteins over several days (Schwanhäusser et al., 2011), this issue has been overcome by combining pulse-labeling MS and tandem-mass tag (TMT)-based multiplexing (Welle et al., 2016). TMT allows isobaric tagging and pooling of up to 11 samples into one multiplexed sample. Combining pulse-labeling with TMT can achieve a balanced distribution of unlabeled and labeled protein species. However, these methods do not allow studying acute processes in response to cellular stimulation, such as cellular stress affecting translation via elF2 $\alpha /$ mTORC1.

Here, we describe multiplexed enhanced protein dynamics (mePROD) MS that allows quantifying heavy label incorporation after very short labeling times without a loss of depth or accuracy. mePROD is based on addition of a booster channel that increases the signal of interest in a TMT-multiplexed and dynamic SILAC-labeled sample. This method enables acute monitoring of global translation rates and captures global translation attenuation by quantifying newly synthesized proteins. Employing mePROD, we provide insight into the global rearrangement of cellular translation upon modulation of elF2 $\alpha$ and/or mTORC 1 activity to reveal common mechanisms in these distinct stress responses.

\section{RESULTS}

mePROD Enables Detecting SILAC Incorporation at Low Stoichiometry

Effects of stress on cellular translation are rapid and occur within a few hours (Prostko et al., 1993). Therefore, time-resolved methods are required to quantify translatome changes upon acute stresses. However, since the median half-life of proteins is about $46 \mathrm{~h}$ (Schwanhäusser et al., 2011), only a small fraction of every protein is to be newly synthesized in the first hours upon cellular modulation. To simulate this situation, we mixed heavy and light peptides at set ratios to assess the capability of pulsed-SILAC to monitor acute changes in translation. Peptides derived from digested HeLa whole-cell lysates grown in light SILAC medium (from here on referred to as light) or in heavy SILAC medium ( $\mathrm{K} 8$ and Arg10 labeled, from here on referred to as heavy) were mixed at ratios ranging from $0.1 \%$ to $10 \%$ heavy/total $(\mathrm{H} / \mathrm{T})$ and analyzed by LC-MS ${ }^{2}$ (Figure 1A). Examining the range of measured $\mathrm{H} / \mathrm{T}$ ratios revealed low accuracy, in particular for low $\mathrm{H} / \mathrm{T}$ ratios, with the measured median for samples mixed at an $\mathrm{H} / \mathrm{T}$ ratio of $0.1 \%$ deviating by about 100 fold from the expected ratio (Figure $1 \mathrm{~B}$ ). At low $\mathrm{H} / \mathrm{T}$ ratios, only 216 peptides were identified, at least partially explaining the high variation observed (Figure 1C). The number of identified peptides increased at higher $\mathrm{H} / \mathrm{T}$ ratios consistent with an inherent $\mathrm{H} / \mathrm{T}$ threshold required for correct quantification of $H / T$ ratios. Thus, as previously described (Schwanhäusser et al., 2011), pulsed SILAC allowed us to monitor relative translation rates; however, for low $\mathrm{H} / \mathrm{T}$ ratios, representing translation activity occurring in the time span of few hours, identification rates and accuracy of quantification was insufficient, since heavy peaks were below the detection limit (Figure 1D, top).

When combining pulsed-SILAC with TMT-labeling, the MS signals of (heavy) peptides sum up across all samples due to the isobaric nature of the TMT tag. We hypothesized that we could take advantage of that property by adding a booster channel containing peptides from fully SILAC-labeled cell lysates. This approach can increase the summed heavy peak intensity across all samples and enable accurate measurement of protein translation at small $\mathrm{H} / \mathrm{T}$ ratios (Figure 1D, bottom). To validate this hypothesis, we prepared a TMT-labeled 10-plex sample containing equimolar amounts of a dilution range of $\mathrm{H} / \mathrm{T}$ ratios (from $0.1 \%-10 \% \mathrm{H} / \mathrm{T}$, as in Figure $1 \mathrm{~A}$ ), a fully SI$\mathrm{LAC}$-labeled cell digest to increase the signal of heavy-labeled $M^{1}$ peaks (booster channel), and a non-SILAC-labeled cell digest (noise channel) to determine noise levels and allow baseline subtraction for individual peptides (Figure 1E). We identified and quantified 1,346 heavy peptides for all channels, improving the identification rate by up to 6 -fold across the range of measured $\mathrm{H} / \mathrm{T}$ ratios (Figure $1 \mathrm{~F}$ ) while using $12.5 \%$ of the machine time necessary for individual SILAC samples (Figures $1 \mathrm{~A}$ and $1 \mathrm{~B}$ ). mePROD correctly determined $\mathrm{H} / \mathrm{T}$ ratios across the whole range (Figure $1 \mathrm{G}$ ) and improved accuracy by three orders of magnitude, especially for lower $\mathrm{H} / \mathrm{T}$ ratios (Figure $1 \mathrm{H}$ ). Together, these results demonstrated the capacity of mePROD to both increase the identification rate of $\mathrm{H} / \mathrm{T}$ ratios and accuracy.

\section{Measuring Translation by mePROD}

We next tested whether increasing the amount of booster channel added could further improve identification rates. Indeed, increasing the amount of booster channel resulted in higher identification rates of heavy SILAC-labeled peptides without affecting overall quantification results (Figures $2 \mathrm{~A}$ and $2 \mathrm{~B}$ ). As the variance of quantification increased 4 - to 5-fold with booster channel levels at or above $300 \%$ (Figure 2C), we continued with using the booster channel at double-molar ratio (200\%).

Next, we analyzed the dynamic range and accuracy of mePROD (Figure 2D). Plotting measured against input ratios showed linear behavior across the whole range with a $R^{2}$ value of 0.998 (Figures $2 \mathrm{E}-2 \mathrm{G}$ ), demonstrating the capability of 
A
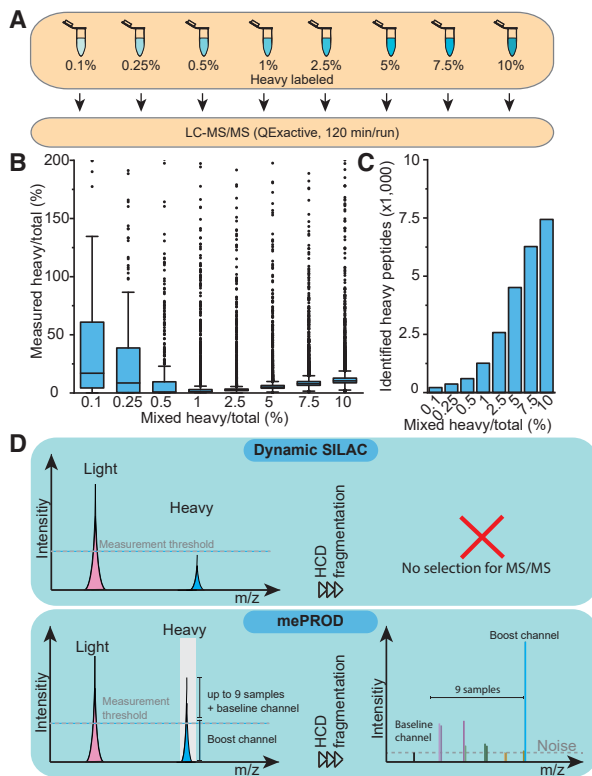
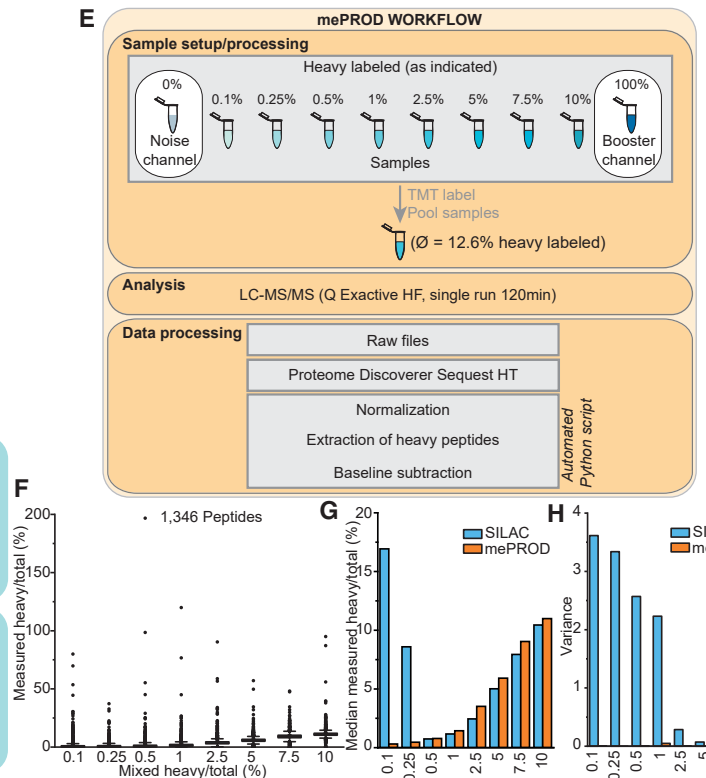

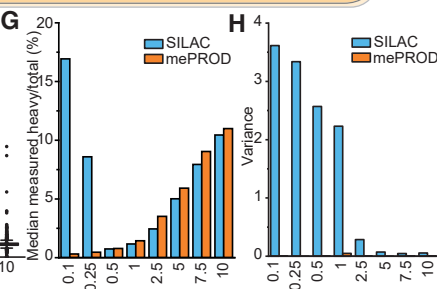

Figure 1. mePROD Proteomics Overcomes Low Accuracy and Identifications of Peptides at Low Heavy-to-Light Ratio (A) Scheme of experimental design. Heavy and light peptides were mixed at indicated ratios.

(B) Measured heavy to total ratios on peptide level. Boxes indicate $25 \% / 50 \%$ quartiles and the median; whiskers show standard deviation. (C) Number of heavy labeled peptides quantified in (B).

(D) Underlying principle of mePROD to increase signals of interest. Low labeling stoichiometry prevents reaching the measurement threshold using standard dynamic SILAC approaches (top). In mePROD, a booster channel comprised of a fully heavy labeled proteome boosts the signal of interest above the MS ${ }^{1}$ detection level (bottom). Heavy/total ratios for individual samples are then then determined from TMT signals quantified in MS $^{2}$ (right).

(E) Experimental mePROD design and data processing. Samples from (A) were combined with noise and booster channels, TMT-labeled, pooled, analyzed by LC-MS ${ }^{2}$, and raw files processed. Reporter ion intensities for peptides were sum normalized and heavy peptide intensities extracted. To enhance accuracy, baseline values derived from the non-SILAC labeled channel were subtracted from each peptide.

(F) Samples as in (A) were analyzed using mePROD (using $1 / 8^{\text {th }}$ of the LC-MS ${ }^{2}$ machine time used in A). Comparison of measured versus expected heavy/total ratios. Boxes indicate $25 \% / 50 \%$ quartiles and the median; whiskers show standard deviation.

$(G$ and $H)$ Comparison of median measured heavy/total peptide ratios $(G)$ or variance $(H)$ for samples measured by SILAC or mePROD. See also Figure S1.

mePROD to accurately measure a wide dynamic range of $\mathrm{H} / \mathrm{T}$ peptide ratios. Comparing $\mathrm{MS}^{2}$ versus $\mathrm{MS}^{3}$ methods did not reveal any major changes (Figure $\mathrm{S} 1 \mathrm{~A}$ ), with the addition of the baseline channel being sufficient to overcome ratio compression (Figure S1B).

To determine the applicability and temporal resolution of mePROD in cells, we labeled HeLa cells for 15-120 min and measured $\mathrm{H} / \mathrm{T}$ ratios (Figure $2 \mathrm{H}$ ), revealing linear behavior $\left(\mathrm{R}^{2}\right.$ of 0.9916 ) and indicating that $15 \mathrm{~min}$ of labeling time was sufficient for quantification (Figures $2 \mathrm{~F}-2 \mathrm{H}, \mathrm{S} 1 \mathrm{C}$, and S1D). To determine the dynamic range of mePROD for translation rate analysis, we inhibited total cellular translation by addition of different concentrations of cycloheximide $(\mathrm{CHX})$ and analyzed the global translation levels. We observed a $\mathrm{CHX}$ concentration dependent decrease in global translation across the full range (Figure 2l). Together, these findings demonstrated that mePROD could determine acute changes in cellular translation with high accuracy.
mePROD MS Quantifies the Functional Translatome upon UPR Induction

Although the unfolded protein response (UPR) causes severe ablation of global translation via phosphorylation of elF2 $\alpha$ (Harding et al., 2000), the precise set of individual proteins, whose translation is reduced upon UPR induction, remains unknown. We determined if mePROD can measure acute changes in translation and identify global translation effects that faithfully reproduce the $\sim 50 \%$ ablation of translation observed by ${ }^{35} \mathrm{~S}$ incorporation experiments (DuRose et al., 2009). Cells were treated in triplicate with DMSO, $1 \mu \mathrm{M}$ thapsigargin, or a co-treatment of $1 \mu \mathrm{M}$ thapsigargin and $500 \mathrm{nM}$ ISRIB (a small molecule reversing the effect of elF2 $\alpha$ phosphorylation (Sidrauski et al., 2015)) and translation measured after $2 \mathrm{~h}$ of label incorporation (Figures 3A and 3B; Table S1). Global translation attenuated by approximately $50 \%$, confirming data observed by other methods (Preston and Hendershot, 2013), and was fully reversed by ISRIB (Figure $3 \mathrm{C}$ ). In addition to detecting global changes in translation, 


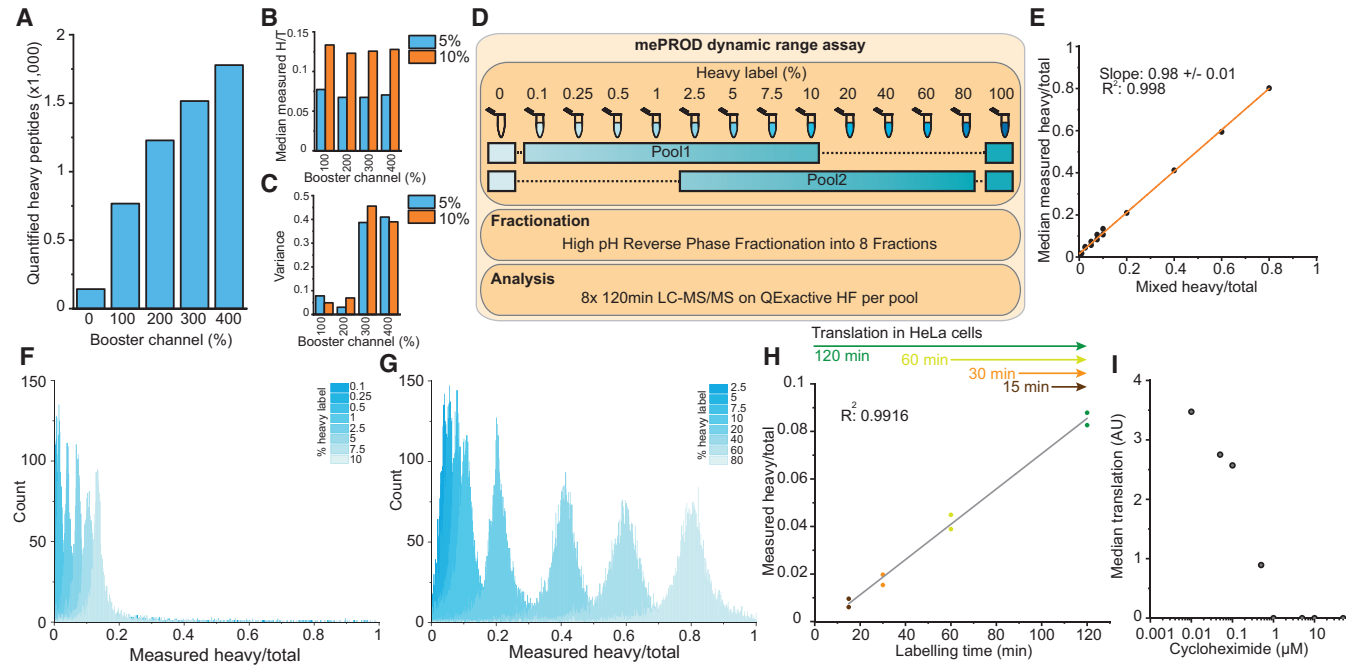

Figure 2. High Dynamic Range of mePROD to Measure Heavy/Light Peptide Ratios and Translation

(A-C) mePROD 6-plex samples were prepared mixing noise channel, two replicates of each $5 \%$ and $10 \%$ heavy/total peptide mix, and indicated amounts (relative to samples) of fully labeled booster channel. Shown are numbers of identified and quantified peptides $(A)$, measured heavy/total $(\mathrm{H} / \mathrm{T}) \mathrm{ratios}(\mathrm{B})$, and variance (C).

(D-G) Experimental design (D). Two mePROD 10-plex samples including samples ranging from $0.1 \%$ to $10 \%$ and $2.5 \%$ to $80 \%$ heavy labeled peptides were mixed with noise and booster channel as indicated, fractionated, and analyzed. Comparison of measured versus expected heavy/total ratios (E). Histograms depicting count distributions of measured heavy/total ratios of 10 -plexes ranging from $0.1 \%-10 \%(F)$ and $2.5 \%-80 \%(G)$.

(H) Measured heavy/total peptide ratios of cells incorporating heavy amino acids into newly synthesized proteins for different lengths of time measured by $\operatorname{mePROD}(n=2)$.

(I) Cells were pre-treated for $2 \mathrm{~h}$ with indicated concentrations of cycloheximide and pulse-labeled for an additional $2 \mathrm{~h}$ with SILAC medium. Median global translation was measured and plotted against cycloheximide concentration. See also Figure S1.

we quantified individual relative translation levels of 5,237 proteins. Multidimensional scaling analysis (MDS) showed replicates clustering together and that samples co-treated with thapsigargin and ISRIB behaved like control samples (Figures 3D and S2). We next investigated proteins displaying significant changes in translation upon UPR induction (adjusted [adj.] $\mathrm{p}$ value $<0.05, \mathrm{FC}(\log 2)<0.5$ or $>0.5)$, when compared to control treatment. Translation of 1,780 proteins was significantly decreased and nine proteins showed increased translation upon UPR induction (Figure 3E). Proteins with increased translation upon UPR contained known UPR targets, such as XBP1 and HERPUD1, that are mediated by the UPR receptor IRE1 and are thus not reliant on eIF2 $\alpha$ and not affected by ISRIB (Lee et al., 2003; Miura et al., 2010; Ron and Walter, 2007; Yoshida et al., 2001) (Figure 3F). Taken together, mePROD can measure acute changes in translation with high overall depth. Strikingly, mePROD translation data strongly overlapped with data derived from ribosome profiling under similar conditions while revealing a much more significant portion of proteins reduced upon UPR induction (Paolini et al., 2018; Reid et al., 2014; Sidrauski et al., 2015) (Figure S3). Moreover, an extensive rearrangement of the cellular translatome upon UPR induction was driven by elF2 $\alpha$, as shown by the nearly complete reversal of translational attenuation when co-treating with ISRIB (Figures 3C, 3D, 3G, and
S4A-S4C). Notably, there was no apparent difference in overall translation ablation of cytosolic versus ER-resident proteins (Figure 3H).

We next sought to analyze the fraction of 623 proteins whose translation did not change upon thapsigargin treatment, suggesting that translation of their mRNAs is resistant to the elF2 $\alpha$ phosphorylation-induced changes observed. GO term enrichment analysis of biological processes showed six significant clusters (Figure 3l). However, the identified clusters overlapped with clusters found for proteins with decreased translation (Figure S4D). This observation strongly suggested that global GO analyses could not explain the observed complexity as subsets of the generalized GO terms appear to be regulated in different ways. Therefore, we analyzed the set of proteins with unchanged translation after thapsigargin treatment on the level of individual proteins using ReactomeFI gene set analysis (Figure $3 \mathrm{~J}$ ). We found 23 different clusters of interacting proteins, with a cluster size larger than two, annotated to different cellular pathways $(q<0.001)$. The identity of those clusters suggests that stress conditions in the ER attenuate global translation while maintaining critical parts of central pathways to maintain cell function. In summary, we could employ mePROD MS to precisely and accurately measure protein translation at high 

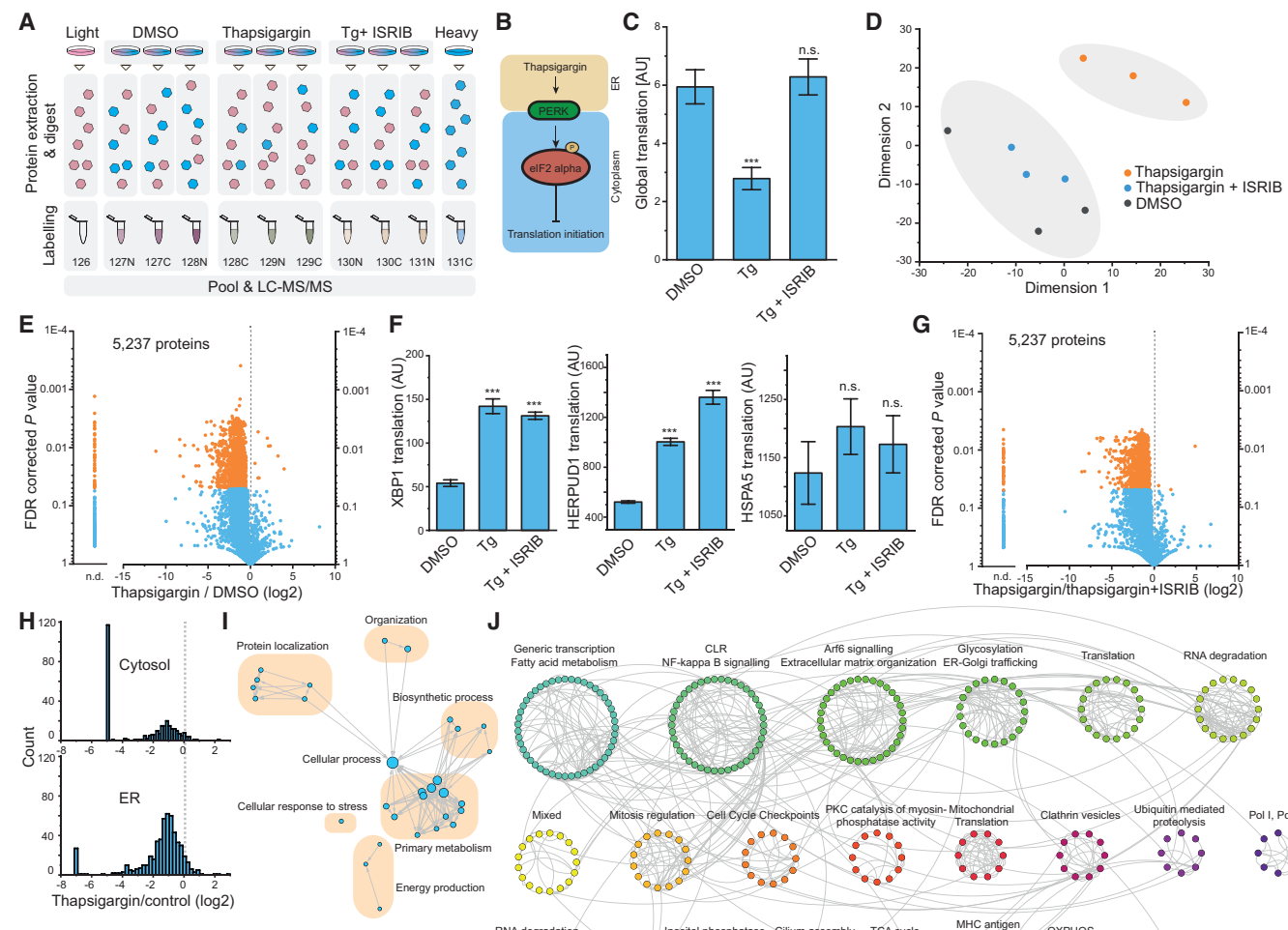

$\mathbf{J}$

$19^{x^{5}}$

Thapsigargin/thapsigargin $+1 S R I B$ (Iog2)

(
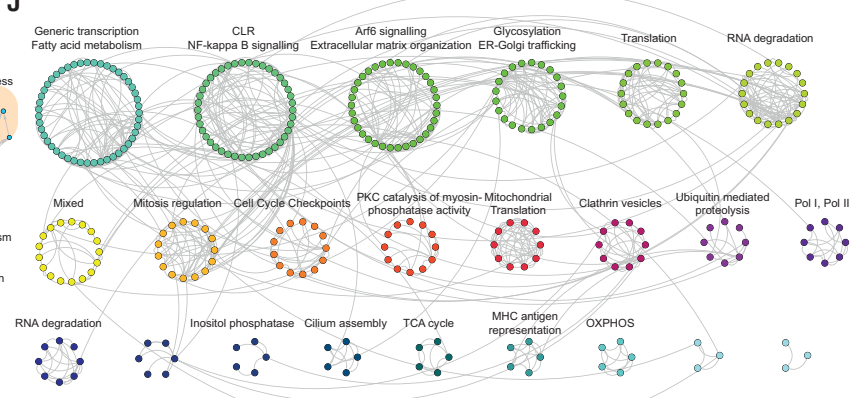

Figure 3. Changes in the Cellular Translatome upon Activation of the Integrated Stress Response by Protein Misfolding in the Endoplasmic Reticulum

(A) Experimental layout. Three different conditions were pooled (in triplicate) with noise and booster channels and analyzed by mePROD MS.

(B) Scheme of translational repression during the UPR, induced by PERK activation.

(C and D) Global translation levels assessed by mePROD MS for cell treated with DMSO, $1 \mu \mathrm{M}$ thapsigargin (Tg), or $1 \mu \mathrm{M}$ thapsigargin and $500 \mathrm{nM}$ ISRIB (Tg + ISRIB) for $2.5 \mathrm{~h}$. Shown are median intensities of heavy labeled peptides (C). Error bars indicate standard deviation $(\mathrm{n}=3)$. ${ }^{\star \star \star} \mathrm{p}<0.001$; $\mathrm{n} . \mathrm{s}$., not significant (twosided, unpaired Student's $t$ test with equal variance). AU, arbitrary units. Multidimensional scaling analysis of samples standardized by unit variance (D).

(E) Volcano plot showing fold change of relative translation versus adjusted $p$ value of thapsigargin versus control treated cells. Orange dots indicate significantly changing proteins ( $p$ values $<0.05$ and fold change [log2] $\leq-0.5$ or $\geq 0.5$ ). Samples for which abundances in thapsigargin treated samples dropped below baseline and no fold change could be calculated are indicated as not determinable (n.d.).

(F) Changes in translation levels of XBP1, HERPUD1, and HSPA5 (better known as BIP) measured by mePROD MS. Mean heavy abundance was plotted with error bars indicating standard deviation $(n=3) .{ }^{\star \star *} p<0.001$; $n$.s., not significant (two-sided, unpaired Student's t test with equal variance). Tg, thapsigargin.

(G) Volcano plot showing fold change versus adjusted $p$ value between thapsigargin and thapsigargin+ISRIB treated samples. Significantly changing proteins in orange (as in E).

(H) Histogram depicting translation changes for cytosolic versus endoplasmic reticulum resident proteins.

(I) EnrichmentMap network showing significantly (q value $<0.001$ ) enriched GO terms for proteins without significantly changed relative translation rates upon thapsigargin treatment.

(J) ReactomeFI cluster analysis for proteins not changing relative translation rates upon thapsigargin treatment. Proteins were $\mathrm{Fl}$ annotated, clustered, and clusters analyzed for significantly enriched Reactome pathways (q value $<0.001$ ). The most prominent pathway of each cluster is indicated. Connecting lines show interaction of protein nodes. See also Table S1 and Figures S2-S4. 


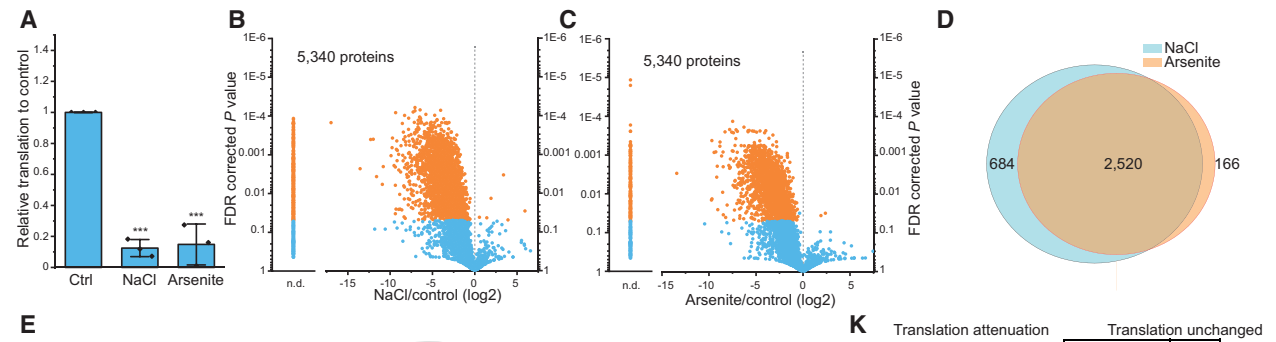

E

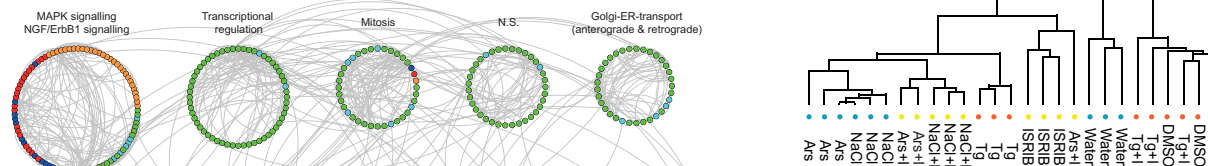

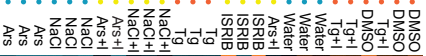

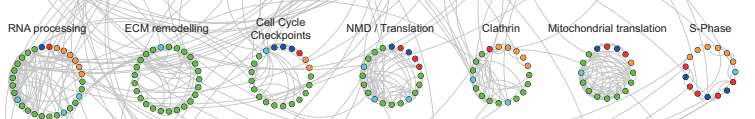

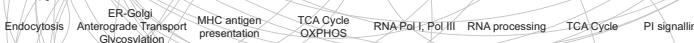

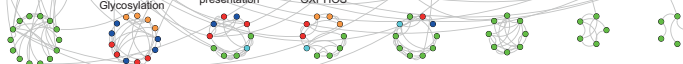

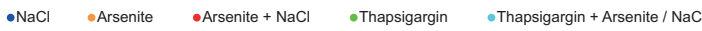

F
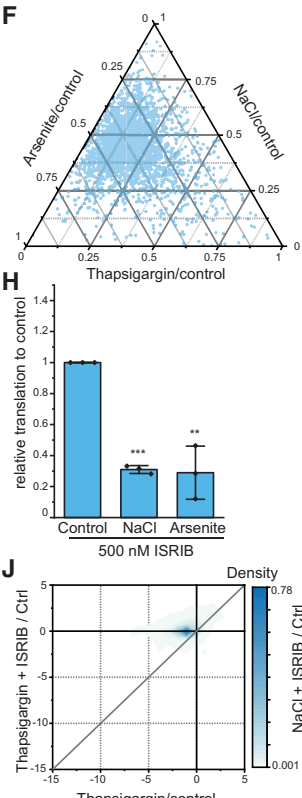

Thapsigargin/control
G Control $\frac{400 \mathrm{mM} \mathrm{NaCl}}{+} \frac{0.5 \mathrm{mM} \text { Arsenite }}{+} 500 \mathrm{nM}$ ISRIB

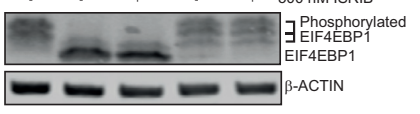

I
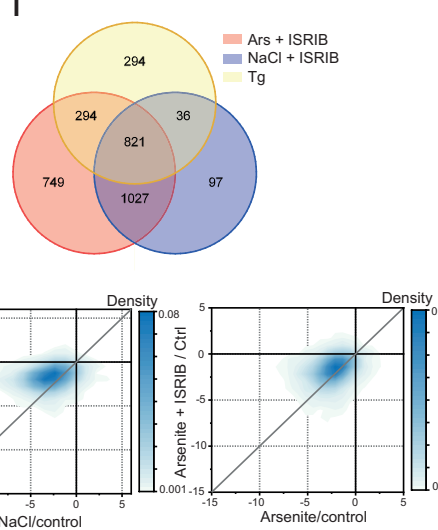

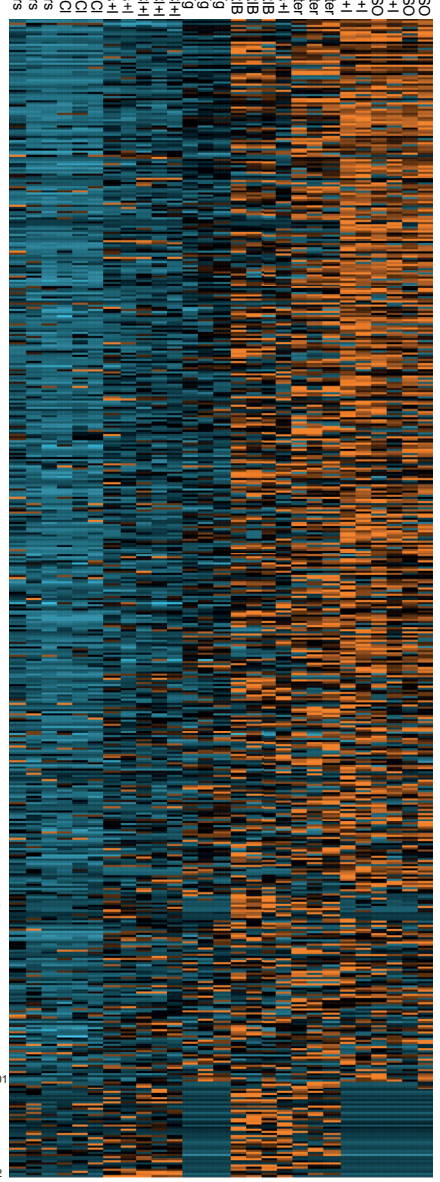

(legend on next page)

918 Molecular Cell 77, 913-925, February 20, 2020 
sensitivity (i.e., below 2 h) to determine the effect of acute thapsigargin treatment on translation.

\section{Different Stress Response Pathways Share Common} Translational Programs

We next asked if diverse ISR activating stressors reshape the cellular translatome in a similar fashion. Therefore, we induced ISR-dependent elF2 $\alpha$ phosphorylation with commonly used treatment paradigms for osmotic or oxidative stress $(400 \mathrm{mM}$ sodium chloride or $500 \mu \mathrm{M}$ arsenite, respectively) (Andreev et al., 2015; Rabouw et al., 2019; Taniuchi et al., 2016) and monitored translation (Figure 4A; Table S2). Consistent with previous studies (Bevilacqua et al., 2010; McEwen et al., 2005), both treatments induced extensive translational attenuation (Fig ure 4A). Quantifying proteins on an individual level showed 3,204 proteins and 2,686 proteins with significant translation decrease for osmotic stress and oxidative stress, respectively (Figures $4 \mathrm{~B}$ and $4 \mathrm{C}$ ), with an overlap of $\sim 87 \%$ (Figure $4 \mathrm{D}$ ). As expected, only few proteins showed increased translation. When comparing the translational effects of these stresses with the ones induced by the UPR, we found distinctively different classes of clusters for specific treatments (Figure 4E): (1) several clusters were exclusive to ER stress. (2) Clusters shared between all three treatments, suggesting a core requirement to maintain cell function. (3) One cluster for ER-to-Golg transport only observed upon $\mathrm{NaCl}$ or arsenite treatment. Strikingly, this cluster was distinct from another ER-to-Golg transport cluster that is specific to thapsigargin treatment, revealing that different subsets of this pathway are sensitive to separate stresses (Figure S4E).

\section{mTORC1 and elF2 $\alpha$ Attenuate Translation of}

Overlapping Protein Sets

Comparing translatomes for the three ISR-inducing stressors showed an overlap of $\sim 30 \%$ of proteins with reduced translation (Figure S5A). Thapsigargin treatment caused a significantly smaller translational effect on individual proteins than the other treatments, suggesting potential differences in translational control (Figure 4F). Indeed, we found $\mathrm{NaCl}$ and arsenite to lead to decreased phosphorylation of the mTORC1 substrate EIF4EBP1 (Figure 4G), consistent with previous publications (Andreev et al., 2015; Plescher et al., 2015). Thus, the observed translatome differences by thapsigargin versus $\mathrm{NaCl}$ or arsenite treatments may be driven by mTORC1. To dissect possible overlapping effects of mTORC1 inhibition and elF2 $\alpha$ phosphorylation, we co-treated cells with $\mathrm{NaCl}$ or arsenite and ISRIB, which we had found to abolish effects seen by elF2 $\alpha$ phosphorylation (Figure 3). As expected, ISRIB had no effect on mTORC1 activity or elF2 $\alpha$ phosphorylation (Figures $4 \mathrm{G}$ and $\mathrm{S} 5 \mathrm{~B})$. However, when monitoring global translation using me$\mathrm{PROD}$, translation repression by $\mathrm{NaCl}$ or arsenite was partially rescued by ISRIB (Figure $4 \mathrm{H}$; Table S3). We compared the fraction of proteins with rescued translation upon ISRIB, as they should be targeted solely by elF2 $\alpha$ (Figure S5C). Surprisingly, we only found a small overlap in this fraction between all three treatments, while proteins still displaying translation attenuation upon ISRIB and $\mathrm{NaCl}$ or arsenite treatment showed a substantial overlap with proteins regulated solely by the ISR/ elF2 $\alpha$ (i.e., seen by thapsigargin treatment, Figure $4 \mathrm{I}$ ). This suggested that elF $2 \alpha$ and mTORC1 might control translation of the same subsets of proteins.

Comparing translation changes of individual proteins following treatment alone or upon co-treatment with ISRIB revealed an increased, but not rescued, translation for the whole population of proteins after ISRIB co-treatment (Figure $4 \mathrm{~J}$ ). The same trend was observed in the global translation behavior (Figures $4 \mathrm{~A}$ and $4 \mathrm{H})$. Clustering analyses further supported these observations showing similar translation patterns of the co-treatments compared to the single treatments (Figure 4K). ISR and mTORC1 modulation also cause transcriptional changes, such as via modulating ATF4 (Park et al., 2017; Ron and Walter, 2007), that could explain overlapping translation changes across the two

Figure 4. Translatome Repression Patterns Shared across Stress Response Pathways

(A) Mean median translation levels of samples treated with water, $400 \mathrm{mM} \mathrm{NaCl}$, or $0.5 \mathrm{mM}$ arsenite for $2.5 \mathrm{~h}$ measured by mePROD MS. Individual values are (A) Mean median translation levels of samples treated with water, $400 \mathrm{mM} \mathrm{NaCl}$, or $0.5 \mathrm{mM}$ arse

( $B$ and $C$ ) Volcano plot showing fold change versus $p$ value for $\mathrm{NaCl}(\mathrm{B})$ or arsenite $(\mathrm{C})$ versus control. Orange dots indicate significantly changing proteins. n.d. not determinable (intensities for treated samples below noise levels).

(D) Overlap between translational repressed proteins (fold change [log2] $<-0.5$ and adj. $p<0.05$ ) in $\mathrm{NaCl}$ or arsenite-treated cells.

(E) ReactomeFI cluster network (q value < 0.001). Unchanged proteins in three treatments (thapsigargin, $\mathrm{NaCl}$, arsenite, fold change [log2] > -0.35 ) were merged into one network, clustered by functional enrichment, and clusters analyzed for reactome pathway enrichment. Proteins were colored according to dataset and most prominent pathways of each cluster annotated. Connecting lines show interaction of protein nodes.

(F) Ternary plot comparing fold changes for each protein between thapsigargin, $\mathrm{NaCl}$, or arsenite treatments. For each protein and treatment, fold changes were summed and ratios to total fold changes determined and plotted.

(G) Western blot showing phosphorylation of EIF4EBP1 upon control, NaCl, or arsenite treatment with or without ISRIB co-treatment. EIF4EBP1 antibody reveals both non-phosphorylated and phosphorylated species.

(H) Cells were treated as in (A) with addition of $500 \mathrm{nM}$ ISRIB. Histogram of global translation relative to control with standard deviation $(n=3) .{ }^{* *} \mathrm{p}<0.01 ;$ ${ }_{\star \star \star *} \mathrm{p}<0.001$ (Two-sided Student's $\mathrm{t}$ test).

(I) Overlap of proteins translationally repressed via elF2 $\alpha$. phosphorylation (by thapsigargin) and proteins not showing reversal by co-treatment with ISRIB and $\mathrm{NaCl}$ and arsenite.

(J) Density plots showing translation fold changes for each protein between stressor alone and co-treatment with ISRIB. Grey lines represent the reference line for equal fold changes.

(K) Heatmap and hierarchical clustering summarizing result for all shown treatments (Figures 3 and 4). Datasets were combined, $Z$ scores calculated, and hierarchical clustering performed using Euclidean distance between the samples. Depicted are $Z$ score values for each treatment and replicate $(n=3)$. Colored circles indicate the 11plex experiment in which the sample was included. I, ISRIB; Ars, arsenite; Tg, thapsigargin. See also Tables S2 and S3 and Figures S4 and S5. 


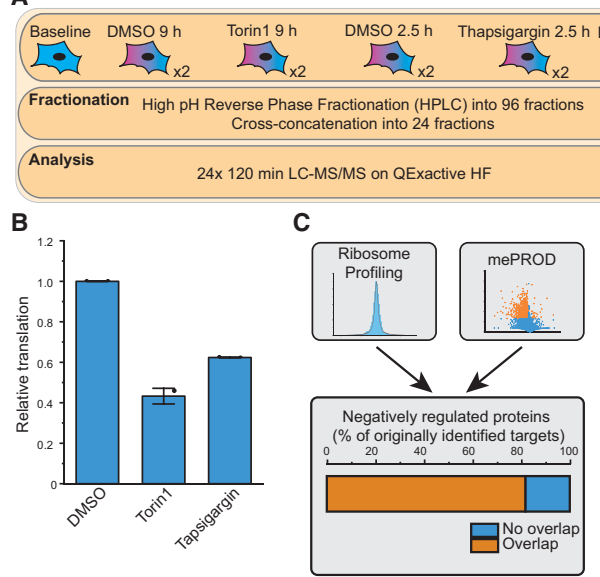

D

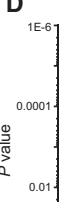
0.01

$\mathbf{F}$

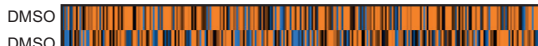
Torin1 Torin1 DMSO

Thapsigargin

Thapsigargin
E
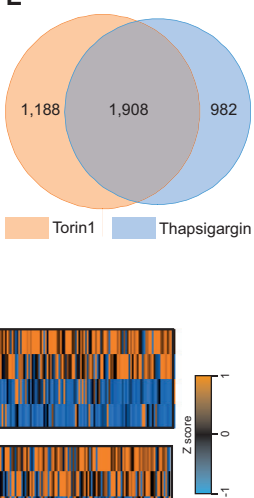

Figure 5. Converging Translatome Regulation by the Integrated Stress Response and mTORC1

(A) Experimental scheme. Cell were treated with thapsigargin or Torin1 for different lengths of time to achieve comparable global translation attenuation (B) Bar plot showing median global translation levels normalized to the respective control with standard deviation $(n=2)$.

(C) Overlap of proteins with reduced relative translation rates upon Torin 1 treatment determined by ribosome profiling data (Thoreen et. al. 2012), or mePROD MS (A). No overlap indicates proteins only showing reduction in ribosome profiling dataset.

(D) Volcano plot showing relative translation changes for Torin1 versus control treated cells plotted against $p$ value $(n=2)$.

(E) Venn diagram displaying the overlap of proteins with reduced relative translation (fold change [log2] $<-0.5$ ).

(F) Heatmap of translation changes for individual treatments and replicates. Data were row-normalized by computing Z scores. See also Table S4.

pathways. However, when comparing published RNA-seq datasets upon ISR activation or mTORC1 inhibition, we found no changes in global or individual transcript changes that could explain the observed translatome rearrangements (Figures S5D-S5F).

These findings indicate that both translational control pathways-ISR and mTORC1-directly regulate translation of the same proteins. This observation was not apparent from previous Ribo-seq analyses. However, it is consistent with the notion that both ISR and mTORC1 control cap-dependent translation initiation, suggesting that translational targets of the two pathways may indeed overlap. Strikingly, our observations (Figure 4J) also suggested a correlation between individual and global protein translation rates.

Intrinsic Features Define mTORC1 and ISR Translation Repression Targets

To further evaluate this hypothesis, we compared translation profiles of cells upon using conditions inhibiting global translation to a similar extent via the ISR (Thapsigargin, $2 \mathrm{~h}$ ) or mTORC1 (Torin1, $9 \mathrm{~h}$ ) in one mePROD sample (Figure 5A). Treatment with Torin1 decreased global translation levels by 59\% (Figure 5B; Table S4), consistent with previous studies observing $\sim 65 \%$ attenuation (Thoreen et al., 2012). Torin1induced translatome differences were largely direct effects on translation, not due to transcriptome changes (Figures S5D and S5G), and showed an $87 \%$ overlap with previously published Ribo-seq data (Figure 5C) (Thoreen et al., 2012).
In addition, mePROD identified over 786 additional, significantly attenuated proteins (Figure 5D). Analyzing the overlap of translationally repressed targets (FC [log2] < - 0.5) in both sample sets, we observed $66 \%$ of proteins controlled by the ISR and mTORC1 alike (Figures 5E and 5F), confirming a high overlap between translation attenuation targets when inhibiting global translation to similar levels. Overall, comparing changes in the translatome upon treatment with thapsigargin or Torin1 confirmed that (1) the majority of translation targets was indeed regulated by both pathways, and (2) target specificity was not achieved by specific activation of the ISR or mTORC1 inhibition. Thus, translation of sets of proteins did not appear to be controlled by the respective extrinsic pathways (i.e., ISR or mTORC1), instead implying intrinsic factors, such as differential sensitivity of mRNA translation to stress, to control individual protein translation. Consistently, we observed translation of some proteins to be more sensitive to global translation attenuation than others, suggesting inherent differences.

Individual Protein Synthesis Levels Correlate with Global Translation Rates under Stress Conditions To validate the hypothesis that translation rates of most individual proteins correlate with global translation rates, we monitored dose-dependent translation attenuation using different concentrations of thapsigargin and Torin1 (Figure 6A). Clustering analysis showed that samples clustered based on global translation attenuation rate rather than on pathway 


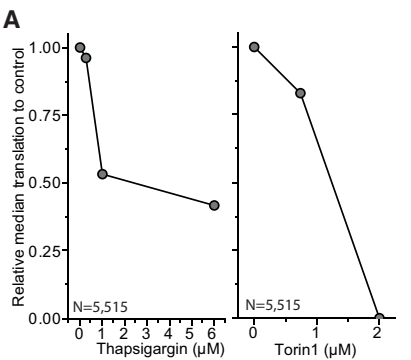

D
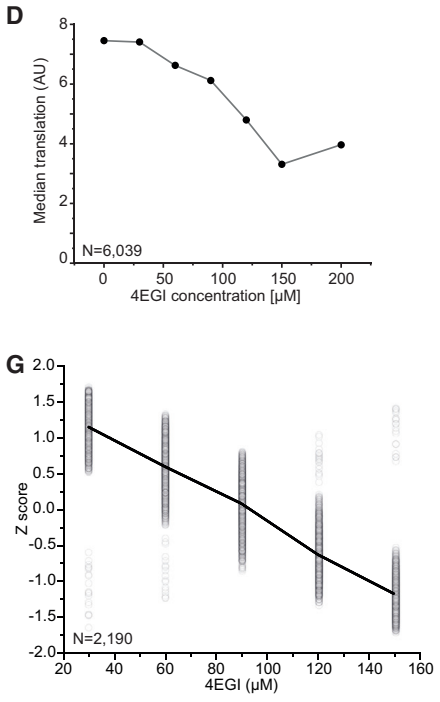

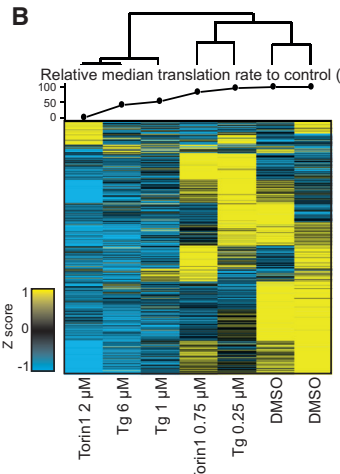

E

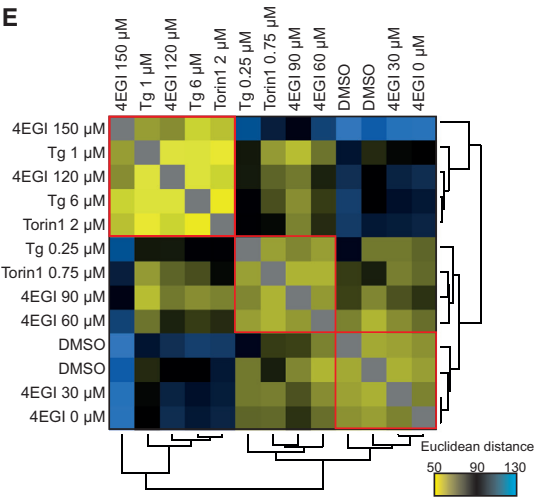

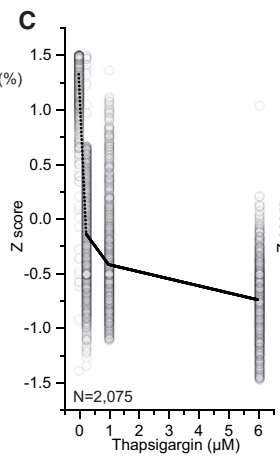

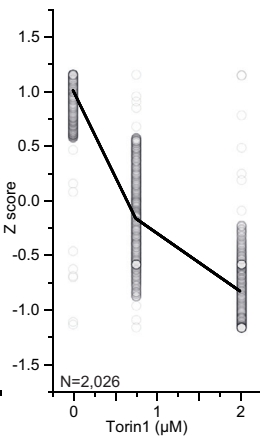

F

(T)

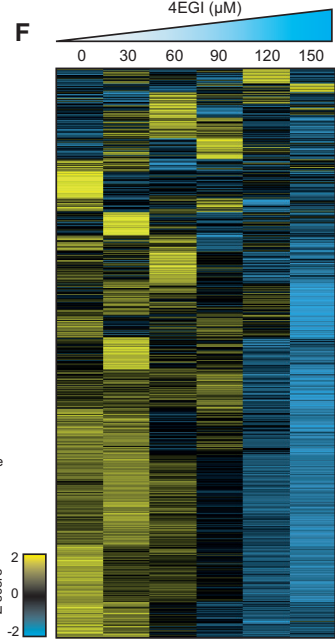

Figure 6. Reduction of Individual Protein Translation Rates Is Defined by the Extent of Global Translation Attenuation

(A) Median relative translation for cells treated with DMSO, $0.25 \mu \mathrm{M}, 1 \mu \mathrm{M}$, or $6 \mu \mathrm{M}$ thapsigargin (Tg) for $2.5 \mathrm{~h}$ (left panel) or DMSO, $0.75 \mu \mathrm{M}$, or $2 \mu \mathrm{M}$ Torin 1 for $9 \mathrm{~h}$ (right panel).

(B) Heatmap showing Z scores of relative translation rates for individual proteins across treatments (Z scores were calculated for each experiment). Clustering of samples were performed with Euclidean distance. Relative median translation rates compared to control are plotted on top of the heatmap for each sample. (C) Standardized (Z score) relative translation rates for the subset of proteins showing a decrease in translation correlating with global translation attenuation after titration of treatments. Clustering was performed on data from (B) and values of the most prominent cluster plotted for each treatment. Black lines indicate averaged curves from all displayed proteins.

(D) Median relative translation rates of cells treated with indicated concentrations of 4EGI.

(E) Heatmap displaying correlation of samples treated with different concentrations of either 4EGl, thapsigargin (Tg) or Torin1. Values represent Euclidean distance between samples. Clustering was performed over Euclidean distance. Apparent clusters are marked in red.

(F) Heatmap displaying standardized relative translation values ( $Z$ score) for individual proteins following 4EGl treatment.

(G) Standardized translation rates (Z score) for all proteins showing linear behavior of translation repression upon 4EGl titration (Figure S6B). See also Figure S6 and Table S5.

(Figure 6B). We next analyzed the behavior of individual proteins after different treatment concentrations (Figure 6C). The biggest clusters of individual proteins followed a similar trend as the global translation (Figures $6 \mathrm{~A}$ and $6 \mathrm{C}$ ).

To evaluate this model on translation level without effects of the upstream pathways (i.e., ISR and mTORC1), we inhibited cap-dependent translation directly, using EIF4E/EIF4G interaction inhibitor 1 (4EGl) (Moerke et al., 2007). Titrating 4EGI caused dose-dependent translation attenuation (Figure 6D; Table S5). Comparing this data to translation inhibition with thapsigargin or Torin1 titration again showed clustering according to the grade of translation inhibition (Figure 6E), not 

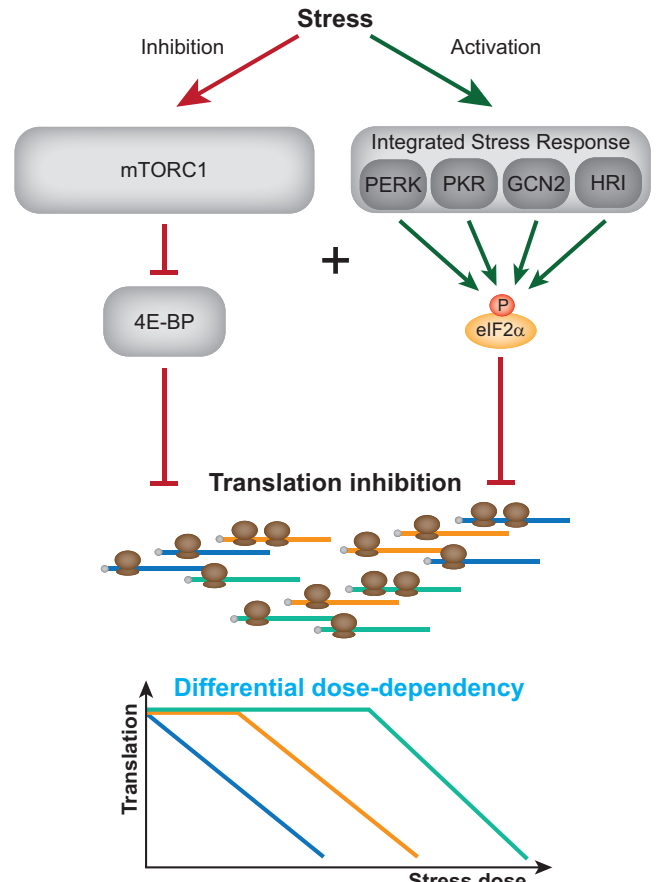

Figure 7. Model of Translation Regulation by mTORC1 and the Integrated Stress Response

Model illustrating that the integrated stress response and mTORC1 regulate translation of an overlapping set of proteins despite their altering upstream translation of an overlapping set of proteins despite their altering upstream
regulation. Translation of individual proteins is largely explained by intrinsic factors with differential sensitivity of global translation inhibition as majo determinant.

inhibitor used, with three apparent major clusters representing different global translation rates. Cluster analysis on the leve of individual proteins showed a major cluster correlating with global translation levels and with a near linear behavior of individual proteins (Figures $6 \mathrm{~F}$ and S6A). Carrying out linear fits across all detected proteins revealed 2,190 proteins following this linear trend (Figure 6G and S6B), demonstrating that translation rates of the majority of cellular proteins directly correlate with global translation attenuation irrespective of the origin of translation attenuation (i.e., ISR or mTORC1). Notably, also these analyses exposed a fraction of proteins evading repression at all examined concentrations, consistent with previously published data describing core cellular pathways to be unaffected by inhibition of cap-dependent translation (Figure S6C) (Marques-Ramos et al., 2017). Together, these results demonstrate that individual protein translation upon stress is controlled by intrinsic factors, largely defining a threshold of global translation attenuation upon which translation of individual proteins ablates (Figure 7).

\section{DISCUSSION}

Determining the transcriptomes and proteomes of cells under various conditions has become a well-established standard used in many biological and medical applications. However, it has become clear that they correlate poorly and that monitoring the translatome as well is essential to understand protein synthesis and the regulation thereof (Ingolia et al., 2012; Maier et al., 2009). Ribo-seq has become the standard method to determine translation rates applied to many biological questions. However, it remains laborious, typically requires large amounts of sample material, and remains expensive (Ingolia et al., 2012; McGlincy and Ingolia, 2017). Thus, for many cellular conditions and stresses, particularly also in primary cells, translatome data are lacking, and its status and regulation is unknown, preventing understanding their role in cellular physiology. Furthermore, ribosome profiling can introduce a bias when measuring translation in states of global repression (Gandin et al., 2016; Masvidal et al., 2017), partially explaining critical differences in conclusions drawn from different experimental setups (Hsieh et al., 2012; Larsson et al., 2012; Morita et al., 2013; Thoreen et al., 2012).

To provide with a proteomics method complementary to Ribo-seq and to overcome some of its challenges, we developed mePROD that offers: (1) high sensitivity, allowing the measurement of highly acute differences in protein synthesis, (2) determination of translatome changes upon conditions with strong global translation shifts without normalization artifacts, and (3) an approach to quantify the translatome with limited sample input (i.e., around 100,000 cells) and at low cost (standard MS protocols and machines). Naturally, proteomic methods, including mePROD, do currently not provide with the same depth as Ribo-seq. Instead, mePROD offers direct information on nascent and newly synthesized proteins that present another layer of information directly related to translation. Due to its simplicity, sensitivity, and low price, mePROD may be applied to numerous biological questions not previously studied or applicable to Ribo-seq.

Key feature of mePROD is the inclusion of a "booster" channel that enables measurement of the signal of interest (newly synthesized peptides) by providing distinctive advantages: first, the booster channel only contains the signal of interest-heavy labeled peptides - thus specifically boosting the signal of newly translated proteins to reliably pass the limit of detection and identification. Second, the booster channel serves as an absolute reference point to allow determining translation relative to the booster channel and enables the comparison of samples analyzed in different LC-MS runs. In addition, mePROD also contains a noise channel comprised of light peptides to determine background noise levels and co-isolation interference for each individual peptide. This makes ratio compression, caused by co-isolation of non-targeted ions, as typically observed in $\mathrm{TMT} \mathrm{MS}^{2}$-based methods, largely negligible. As a result, mePROD data acquisition can be carried out with $\mathrm{MS}^{2}$ methods, offering higher sensitivity and identification and quantification rates (Figures S1A and S1B). Together, mePROD enables translation proteomics with a temporal resolution capable of examining short-term 
changes of relative translation rates, as seen during stress responses.

mePROD offers various advantages for global translation quantification: (1) direct quantification of nascent chains, not relying on indirect sequence information, and (2) low input requirements in the range of typical proteomics experiments ( $<100,000$ cells) without the need of ribosome purification Thus, mePROD is especially suitable for setups with limited starting material, such as clinical samples or primary cells. (3) No normalization bias, allowing ready quantification of individual and global protein translation rates, even in situations with global translation defects. At the same time, there are also methodinherent disadvantages driven by the use of mass spectrometry as a readout including an imperfect coverage or lack of detectability of proteins (due to sequence, abundance, and physica properties of peptides) and its limited depth, when compared to NGS based methods, where coverage mainly is a scalable function of sequencing depth. In addition, mePROD does not provide information on ribosome occupancy.

Despite eIF2 $\alpha$ and EIF4EBP1-and thus the ISR and mTORC1-affecting processes in cap-dependent translation, it was generally assumed that the translational targets of elF2a and EIF4EBP1 differ (Wengrod and Gardner, 2015). This is largely due to previous ribosome profiling analyses only uncovering small subsets of mRNAs with decreased translation that showed only minimal overlap between ISR and mTORC1 targets (Hsieh et al., 2012; Sidrauski et al., 2015; Thoreen et al., 2012). However, mePROD revealed the full extent of the extensive remodeling of the translational landscape upon stress induction (Figures 3 and 4). Comparison with previous datasets showed that mePROD identified most translationally regulated proteins revealed by ribosome profiling (Figures 5 and S3). It also detected the remodeling of translation in greater depth, resembling the global changes seen by ${ }^{35} \mathrm{~S}-$ Met metabolic labeling. By analyzing the detailed sets of translationally repressed proteins, we found that both pathways-ISR and mTORC1-have converging sets of targets (Figure 5). Crosstalk between both pathways is emerging as an interesting concept (Nikonorova et al., 2018; Zhang et al., 2018) in recent years, pointing to a complex picture of stress responses driving translational and transcriptional control. Nevertheless, the vast majority of translational changes cannot be explained by transcriptiona patterns, since previous RNA-sequencing experiments did not show major effects when compared to our data (Figures S5DS5G) (Andreev et al., 2015; Paolini et al., 2018; Thoreen et al., 2012).

We found the set of repressed target proteins to be determined by the strength of global translation repression rather than by the upstream pathway activated (Figures 4 and 6). In agreement with this hypothesis, titrating either the stressinducing agents or a cap-dependent translation inhibito showed dose-dependent effects on translation for the majority of proteins (Figure 6). Thus, features of each individual messenger RNA may reflect their sensitivity to translational changes (Figure 7). This model explained the vast majority of changes in the translatome upon modulating global translation. Strikingly, there is a small fraction of proteins not following this pattern, likely controlled by alternative translation initiation, or the specific transcriptional changes brought about by the ISR or mTORC1. These proteins include clusters of core cellular functions to retain their translation upon stress induction (Figures $3 \mathrm{~J}, 4 \mathrm{E}, \mathrm{S} 4$, and $\mathrm{S} 6 \mathrm{C}$ ). Consistent with previously published data (Marques-Ramos et al., 2017), we also found core signaling pathways to be maintained, most prominently the phosphateinositol pathway and the mTOR pathway (Figure S6C). This might play a major role in cellular response to stresses that will result in a shut-down of protein translation of various subsets of proteins, dependent on the extent of stress (i.e., global translation attenuation), while keeping core pathways intact to ensure survival and function of cells during and after recovery from stress.

\section{STAR $\star$ METHODS}

Detailed methods are provided in the online version of this paper and include the following:

- KEY RESOURCES TABLE

- LEAD CONTACT AND MATERIALS AVAILABILITY

- EXPERIMENTAL MODEL AND SUBJECT DETAILS O Cell lines and culture conditions

- METHOD DETAILS

○ Cell harvest and lysis

O Sample preparation for LC-MS ${ }^{2}$

o High-pH Reverse Phase fractionation

O Mass spectrometry

Western Blotting

- QUANTIFICATION AND STATISTICAL ANALYSIS

- Processing of raw files

$\circ$ Data Analysis and statistics

Multidimensional scaling

Hierarchical clustering

O Network analysis

- DATA AND CODE AVAILABILITY

SUPPLEMENTAL INFORMATION

Supplemental Information can be found online at https://doi.org/10.1016/ molcel.2019.11.010

\section{ACKNOWLEDGMENTS}

We thank Ivan Dikic, Stefan Müller, and Tobias Schmid for helpful comments on the manuscript. C.M. was supported by the European Research Council under the European Union's Seventh Framework Programme (ERC StG 803565), the Emmy Noether Program of the Deutsche Forschungsgemeinschaft (DFG, MU 4216/1-1), and the Johanna Quandt Young Academy at Goethe.

\section{AUTHOR CONTRIBUTIONS}

K.K. carried out all experiments and analyzed data. K.K. and G.T. prepared samples, performed mass spectrometry analyses, and discussed the data. K.K. and C.M. designed experiments, interpreted data, and wrote the manuscript. C.M. conceived and supervised the study.

DECLARATION OF INTERESTS

The authors declare no competing interests. 
Received: June 26, 2019

Revised: September 24, 2019

Accepted: November 7, 2019

Published: December 4, 2019

\section{REFERENCES}

Andreev, D.E., O'Connor, P.B., Fahey, C., Kenny, E.M., Terenin, I.M., Dmitriev, S.E., Cormican, P., Morris, D.W., Shatsky, I.N., and Baranov, P.V. (2015) Translation of $5^{\prime}$ leaders is pervasive in genes resistant to elF2 repression. eLife 4, e03971.

Back, S.H., Scheuner, D., Han, J., Song, B., Ribick, M., Wang, J., Gildersleeve, R.D., Pennathur, S., and Kaufman, R.J. (2009). Translation attenuation through elF2 $\alpha$. phosphorylation prevents oxidative stress and maintains the differentiated state in $\beta$ cells. Cell Metab. 10, 13-26.

Becher, I., Andrés-Pons, A., Romanov, N., Stein, F., Schramm, M., Baudin, F. Helm, D., Kurzawa, N., Mateus, A., Mackmull, M.-T., et al. (2018). Pervasiv Protein Thermal Stability Variation during the Cell Cycle. Cell 173, 14951507.e18.

Bevilacqua, E., Wang, X., Majumder, M., Gaccioli, F., Yuan, C.L., Wang, C. Zhu, X., Jordan, L.E., Scheuner, D., Kaufman, R.J., et al. (2010). elF2 $\alpha$ phosphorylation tips the balance to apoptosis during osmotic stress. J. Biol. Chem. 285, 17098-17111.

Chen, K., Hu, Z, Xia, Z., Zhao, D., Li, W., and Tyler, J.K. (2015). The Overlooked Fact: Fundamental Need for Spike-In Control for Virtually All Genome-Wide Analyses. Mol. Cell. Biol. 36, 662-667.

Clavarino, G., Cláudio, N., Couderc, T., Dalet, A., Judith, D., Camosseto, V. Schmidt, E.K., Wenger, T., Lecuit, M., Gatti, E., and Pierre, P. (2012) Induction of GADD34 is necessary for dsRNA-dependent interferon- $\beta$ production and participates in the control of Chikungunya virus infection. PLoS Pathog. 8, e1002708.

Colina, R., Costa-Mattioli, M., Dowling, R.J.O., Jaramillo, M., Tai, L.-H. Breitbach, C.J., Martineau, Y., Larsson, O., Rong, L., Svitkin, Y.V., et al. (2008). Translational control of the innate immune response through IRF-7. Nature 452, 323-328.

Cox, J., and Mann, M. (2008). MaxQuant enables high peptide identification rates, individualized p.p.b.-range mass accuracies and proteome-wide protein quantification. Nat. Biotechnol. 26, 1367-1372.

De Benedetti, A., and Graff, J.R. (2004). elF-4E expression and its role in malignancies and metastases. Oncogene 23, 3189-3199.

Dowling, R.J.O., Topisirovic, I., Alain, T., Bidinosti, M., Fonseca, B.D. Petroulakis, E., Wang, X., Larsson, O., Selvaraj, A., Liu, Y., et al. (2010) mTORC1-mediated cell proliferation, but not cell growth, controlled by the 4E-BPs. Science 328, 1172-1176.

DuRose, J.B., Scheuner, D., Kaufman, R.J., Rothblum, L.I., and Niwa, M. (2009). Phosphorylation of eukaryotic translation initiation factor $2 \alpha$ coordinates rRNA transcription and translation inhibition during endoplasmic reticulum stress. Mol. Cell. Biol. 29, 4295-4307.

Gandin, V., Masvidal, L., Hulea, L., Gravel, S.-P., Cargnello, M. McLaughlan, S., Cai, Y., Balanathan, P., Morita, M., Rajakumar, A., et al. (2016). nanoCAGE reveals $5^{\prime}$ UTR features that define specific modes of translation of functionally related MTOR-sensitive mRNAs. Genome Res. 26, 636-648.

Graff, J.R., and Zimmer, S.G. (2003). Translational control and metastatic progression: enhanced activity of the mRNA cap-binding protein elF-4E selectively enhances translation of metastasis-related mRNAs. Clin. Exp. Metastasis 20, 265-273.

Harding, H.P., Novoa, I., Zhang, Y., Zeng, H., Wek, R., Schapira, M., and Ron, D. (2000). Regulated translation initiation controls stress-induced gene expression in mammalian cells. Mol. Cell 6, 1099-1108.

Holz, M.K., Ballif, B.A., Gygi, S.P., and Blenis, J. (2005). mTOR and S6K mediate assembly of the translation preinitiation complex through dynamic protein interchange and ordered phosphorylation events. Cell 123, 569-580. Hsieh, A.C., Liu, Y., Edlind, M.P., Ingolia, N.T., Janes, M.R., Sher, A., Shi, E.Y., Stumpf, C.R., Christensen, C., Bonham, M.J., et al. (2012). The translational landscape of mTOR signalling steers cancer initiation and metastasis. Nature 485, 55-61.

Hunter, J.D. (2007). Matplotlib: A 2D Graphics Environment. Comput. Sci. Eng 9, 90-95.

Ingolia, N.T., Brar, G.A., Rouskin, S., McGeachy, A.M., and Weissman, J.S. (2012). The ribosome profiling strategy for monitoring translation in vivo by deep sequencing of ribosome-protected mRNA fragments. Nat. Protoc. 7 1534-1550.

Jiang, Z., Yang, J., Dai, A., Wang, Y., Li, W., and Xie, Z. (2017). Ribosome profiling reveals translational regulation of mammalian cells in response to hypoxic stress. BMC Genomics 18, 638.

Jovanovic, M., Rooney, M.S., Mertins, P., Przybylski, D., Chevrier, N., Satija, R. Rodriguez, E.H., Fields, A.P., Schwartz, S., Raychowdhury, R., et al. (2015). Immunogenetics. Dynamic profiling of the protein life cycle in response to pathogens. Science 347, 1259038.

Kozak, M. (1999). Initiation of translation in prokaryotes and eukaryotes. Gene 234, 187-208.

Krishnamoorthy, T., Pavitt, G.D., Zhang, F., Dever, T.E., and Hinnebusch, A.G (2001). Tight binding of the phosphorylated $\alpha$ subunit of initiation factor 2 $(\mathrm{elF} 2 \alpha)$ to the regulatory subunits of guanine nucleotide exchange factor elF2B is required for inhibition of translation initiation. Mol. Cell. Biol. 21, 5018-5030.

Larsson, O., Morita, M., Topisirovic, I., Alain, T., Blouin, M.-J., Pollak, M., and Sonenberg, N. (2012). Distinct perturbation of the translatome by the antidiabetic drug metformin. Proc. Natl. Acad. Sci. U. S. A. 109, 8977-8982.

Lee, A.-H., Iwakoshi, N.N., and Glimcher, L.H. (2003). XBP-1 regulates a subset of endoplasmic reticulum resident chaperone genes in the unfolded protein response. Mol. Cell. Biol. 23, 7448-7459.

Maere, S., Heymans, K., and Kuiper, M. (2005). BiNGO: a Cytoscape plugin to assess overrepresentation of gene ontology categories in biological networks. Bioinformatics 21, 3448-3449.

Maier, T., Güell, M., and Serrano, L. (2009). Correlation of mRNA and protein in complex biological samples. FEBS Lett. 583, 3966-3973.

Marques-Ramos, A., Candeias, M.M., Menezes, J., Lacerda, R., Willcocks, M. Teixeira, A., Locker, N., and Romão, L. (2017). Cap-independent translation ensures mTOR expression and function upon protein synthesis inhibition. RNA 23, 1712-1728.

Masvidal, L., Hulea, L., Furic, L., Topisirovic, I., and Larsson, O. (2017). mTORsensitive translation: Cleared fog reveals more trees. RNA Biol. 14, 1299-1305. Mathieson, T., Franken, H., Kosinski, J., Kurzawa, N., Zinn, N., Sweetman, G., Poeckel, D., Ratnu, V.S., Schramm, M., Becher, I., et al. (2018). Systematic analysis of protein turnover in primary cells. Nat. Commun. 9, 689 .

McEwen, E., Kedersha, N., Song, B., Scheuner, D., Gilks, N., Han, A., Chen, J.J., Anderson, P, and Kaufman, RJ. (2005). Heme-regulated inhibitor ki-

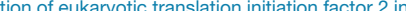
hibits translation, induces stress granule formation, and mediates survival upon arsenite exposure. J. Biol. Chem. 280, 16925-16933.

McGlincy, N.J., and Ingolia, N.T. (2017). Transcriptome-wide measurement of translation by ribosome profiling. Methods 126, 112-129.

McKinney, W. (2010). Data Structures for Statistical Computing in Python. Proceedings of the 9th Python in Science Conference, 51-56.

Merico, D., Isserlin, R., Stueker, O., Emili, A., and Bader, G.D. (2010). Enrichment map: a network-based method for gene-set enrichment visualization and interpretation. PLoS ONE 5, e13984.

Miura, H., Hashida, K., Sudo, H., Awa, Y., Takarada-lemata, M., Kokame, K. Takahashi, T., Matsumoto, M., Kitao, Y., and Hori, O. (2010). Deletion of Herp facilitates degradation of cytosolic proteins. Genes Cells 15, 843-853.

Moerke, N.J., Aktas, H., Chen, H., Cantel, S., Reibarkh, M.Y., Fahmy, A. Gross, J.D., Degterev, A., Yuan, J., Chorev, M., et al. (2007). Small-molecule inhibition of the interaction between the translation initiation factors elF4E and elF4G. Cell 128, 257-267.

924 Molecular Cell 77, 913-925, February 20, 2020 


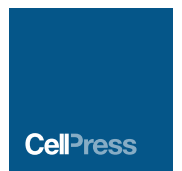

Morita, M., Gravel, S.-P., Chénard, V., Sikström, K., Zheng, L., Alain, T. Gandin, V., Avizonis, D., Arguello, M., Zakaria, C., et al. (2013). mTORC1 controls mitochondrial activity and biogenesis through $4 \mathrm{E}-\mathrm{BP}$-dependent translational regulation. Cell Metab. 18, 698-711.

Münch, C., and Harper, J.W. (2016). Mitochondrial unfolded protein response controls matrix pre-RNA processing and translation. Nature 534, 710-713.

Nikonorova, I.A., Mirek, E.T., Signore, C.C., Goudie, M.P., Wek, R.C., an Anthony, T.G. (2018). Time-resolved analysis of amino acid stress identifies elF2 phosphorylation as necessary to inhibit mTORC1 activity in liver. J. Biol. Chem. 293, 5005-5015.

Pakos-Zebrucka, K., Koryga, I., Mnich, K., Ljujic, M., Samali, A., and Gorman, A.M. (2016). The integrated stress response. EMBO Rep. 17, 1374-1395.

Paolini, N.A., Moore, K.S., di Summa, F.M., Fokkema, I.F.A.C., 't Hoen, P.A.C. and von Lindern, M. (2018). Ribosome profiling uncovers selective mRNA translation associated with elF2 phosphorylation in erythroid progenitors. PLoS ONE 13, e0193790.

Park, Y., Reyna-Neyra, A., Philippe, L., and Thoreen, C.C. (2017). mTORC Balances Cellular Amino Acid Supply with Demand for Protein Synthesis through Post-transcriptional Control of ATF4. Cell Rep. 19, 1083-1090.

Pedregosa, F., Varoquaux, G., Gramfort, A., Michel, V., Thirion, B., Grisel, O. Blondel, M., Prettenhofer, P. Weiss, R., Dubourg, V., et al. (2011). Scikit-learn: Machine Learning in Python. J. Mach. Learn. Res. 12, 2825-2830.

Perez-Riverol, Y., Csordas, A., Bai, J., Bernal-Llinares, M., Hewapathirana, S. Kundu, D.J., Inuganti, A., Griss, J., Mayer, G., Eisenacher, M., et al. (2019). The PRIDE database and related tools and resources in 2019: improving support for quantification data. Nucleic Acids Res. 47 (D1), D442-D450.

Plescher, M., Teleman, A.A., and Demetriades, C. (2015). TSC2 mediates hy perosmotic stress-induced inactivation of mTORC1. Sci. Rep. 5, 13828.

Preston, A.M., and Hendershot, L.M. (2013). Examination of a second node o translational control in the unfolded protein response. J. Cell Sci. 126, 4253-4261.

Prostko, C.R., Brostrom, M.A., and Brostrom, C.O. (1993). Reversible phosphorylation of eukaryotic initiation factor $2 \alpha$ in response to endoplasmic reticular signaling. Mol. Cell. Biochem. 127-128, 255-265.

Rabouw, H.H., Langereis, M.A., Anand, A.A., Visser, L.J., de Groot, R.J. Walter, P., and van Kuppeveld, F.J.M. (2019). Small molecule ISRIB suppresses the integrated stress response within a defined window of activation. Proc. Natl. Acad. Sci. USA 116, 2097-2102.

Raught, B., Peiretti, F., Gingras, A.-C., Livingstone, M., Shahbazian, D., Mayeur, G.L., Polakiewicz, R.D., Sonenberg, N., and Hershey, J.W. (2004) Phosphorylation of eucaryotic translation initiation factor 4B Ser422 is modulated by S6 kinases. EMBO J. 23, 1761-1769.

Reid, D.W., Chen, Q., Tay, A.S.-L., Shenolikar, S., and Nicchitta, C.V. (2014). The unfolded protein response triggers selective mRNA release from the endoplasmic reticulum. Cell 158, 1362-1374.

Ron, D., and Walter, P. (2007). Signal integration in the endoplasmic reticulum unfolded protein response. Nat. Rev. Mol. Cell Biol. 8, 519-529.

Roux, P.P., and Topisirovic, I. (2012). Regulation of mRNA translation by signaling pathways. Cold Spring Harb. Perspect. Biol. 4, 1-23.

Sabatini, D.M. (2006). mTOR and cancer: insights into a complex relationship. Nat. Rev. Cancer 6, 729-734.
Savitski, M.M., Zinn, N., Faelth-Savitski, M., Poeckel, D., Gade, S., Becher, I., Muelbaier, M Wagner, A. Strohmer, K Werner, T., et al. (2018) Multiplexed Proteome Dynamics Profiling Reveals Mechanisms Controlling Protein Homeostasis. Cell 173, 260-274.e25.

Schwanhäusser, B., Gossen, M., Dittmar, G., and Selbach, M. (2009). Global analysis of cellular protein translation by pulsed SILAC. Proteomics 9 , 205-209.

Schwanhäusser, B., Busse, D., Li, N., Dittmar, G., Schuchhardt, J., Wolf, J., Chen, W., and Selbach, M. (2011). Global quantification of mammalian gene expression control. Nature 473, 337-342.

Shannon, P., Markiel, A., Ozier, O., Baliga, N.S., Wang, J.T., Ramage, D., Amin, N., Schwikowski, B., and Ideker, T. (2003). Cytoscape: a software environment for integrated models of biomolecular interaction networks. Genome Res. 13, 2498-2504.

Sidrauski, C., McGeachy, A.M., Ingolia, N.T., and Walter, P. (2015). The smal molecule ISRIB reverses the effects of elF2 $\alpha$, phosphorylation on translation and stress granule assembly. eLife 4, e05033.

Sonenberg, N., and Hinnebusch, A.G. (2009). Regulation of translation initiation in eukaryotes: mechanisms and biological targets. Cell 136, 731-745.

Taniuchi, S., Miyake, M., Tsugawa, K., Oyadomari, M., and Oyadomari, S. (2016). Integrated stress response of vertebrates is regulated by four elF2 $2 \alpha \mathrm{ki}-$ nases. Sci. Rep. 6, 32886

Thoreen, C.C., Chantranupong, L., Keys, H.R., Wang, T., Gray, N.S., and Sabatini, D.M.M. (2012). A unifying model for mTORC1-mediated regulation of mRNA translation. Nature 485, 109-113.

Tyanova, S., Temu, T., Sinitcyn, P., Carlson, A., Hein, M.Y., Geiger, T., Mann, M., and Cox, J. (2016). The Perseus computational platform for comprehensive analysis of (prote)omics data. Nat. Methods 13, 731-740.

van der Walt, S., Colbert, S.C., and Varoquaux, G. (2011). The NumPy Array: A Structure for Efficient Numerical Computation. Comput. Sci. Eng. 13, 22-30. Wang, X., Li, W., Williams, M., Terada, N., Alessi, D.R., and Proud, C.G. (2001). Regulation of elongation factor 2 kinase by p90(RSK1) and p70 S6 kinase. EMBO J. 20, 4370-4379.

Welle, K.A., Zhang, T., Hryhorenko, J.R., Shen, S., Qu, J., and Ghaemmaghami, S. (2016). Time-resolved Analysis of Proteome Dynamics by Tandem Mass Tags and Stable Isotope Labeling in Cell Culture (TMTSILAC) Hyperplexing. Mol. Cell. Proteomics 15, 3551-3563.

Wengrod, J.C., and Gardner, L.B. (2015). Cellular adaptation to nutrient deprivation: crosstalk between the MTORC1 and elF2 $\alpha$ signaling pathways and implications for autophagy. Cell Cycle 14, 2571-2577.

Wu, G., and Haw, R. (2017). Functional Interaction Network Construction and Analysis for Disease Discovery (New York, NY: Humana Press), pp. 235-253. Yoshida, H., Matsui, T., Yamamoto, A., Okada, T., and Mori, K. (2001). XBP1 mRNA is induced by ATF6 and spliced by IRE1 in response to ER stress to produce a highly active transcription factor. Cell 107, 881-891.

Zhang, S., Macias-Garcia, A., Velazquez, J., Paltrinieri, E., Kaufman, R.J., and Chen, J.-J. (2018). HRI coordinates translation by elF2 $\alpha \mathrm{P}$ and mTORC1 to mitigate ineffective erythropoiesis in mice during iron deficiency. Blood 131 450-461. 


\section{STAR $\star$ METHODS}

\section{KEY RESOURCES TABLE}

\begin{tabular}{|c|c|c|}
\hline REAGENT or RESOURCE & SOURCE & IDENTIFIER \\
\hline \multicolumn{3}{|l|}{ Antibodies } \\
\hline ACTB & SantaCruz & Cat\#sc-69879; RRID:AB_1119529 \\
\hline EIF4EBP1 & Cell Signaling Technologies & Cat\#9644; RRID:AB_2097841 \\
\hline EIF2S1 & Abcam & Cat\#ab5369; RRID:AB_304838 \\
\hline p-EIF2S1(S51) & Abcam & Cat\#ab32157; RRID:AB_732117 \\
\hline IRDye 680RD anti-mouse & Li-Cor & Cat\# 925-68070; RRID:AB_2651128 \\
\hline IRDye $800 \mathrm{CW}$ anti-rabbit & Li-Cor & Cat\# 925-32211; RRID:AB_2651127 \\
\hline \multicolumn{3}{|c|}{ Chemicals, Peptides, and Recombinant Proteins } \\
\hline Thapsigargin & Abcam & Cat\#ab120286 \\
\hline 2-Chloracetamide & Sigma Aldrich & Cat\#C0267 \\
\hline Arginine 10 & Cambridge Isotope Laboratories & Cat\#CNLM-539-H-PK \\
\hline Lysine 8 & Cambridge Isotope Laboratories & Cat\#CNLM-291-H-PK \\
\hline Sodium (meta)arsenite & Sigma Aldrich & Cat\#S7400 \\
\hline ISRIB & Sigma Aldrich & Cat\#SML0843 \\
\hline $4 \mathrm{EGI}-1$ & Selleckchem & Cat\#S7369 \\
\hline TMT reagents & Thermo Fisher Scientific & Cat\#90111, Cat\#A37724, Cat\#90061 \\
\hline Torin1 & Cell Signaling Technologies & Cat\#14379 \\
\hline \multicolumn{3}{|l|}{ Critical Commercial Assays } \\
\hline$\mu \mathrm{BCA}$ microplate assay & Thermo Fisher Scientific & Cat\#23235 \\
\hline \multicolumn{3}{|l|}{ Deposited Data } \\
\hline RAW and quantified data & This paper/PRIDE & PXD014377 and PXD015438 \\
\hline Ribosome profiling and RNaseq data & $\begin{array}{l}\text { Thoreen et.al. 2012, Reid et.al. 2014, } \\
\text { Paolini et.al. 2018, } \\
\text { Andreev et.al. } 2015\end{array}$ & $\begin{array}{l}\text { https://doi.org/10.1038/nature11083, } \\
\text { https://doi.org/10.1016/j.cell.2014.08.012, } \\
\text { https://doi.org/10.1371/journal.pone.0193790, } \\
\text { https://doi.org/10.7554/eLife.0397 }\end{array}$ \\
\hline SwissProt Human protein database & SwissProt & https://www.uniprot.org/downloads \\
\hline \multicolumn{3}{|l|}{ Experimental Models: Cell Lines } \\
\hline Human: HeLa & & $\mathrm{N} / \mathrm{A}$ \\
\hline \multicolumn{3}{|l|}{ Software and Algorithms } \\
\hline Python 3.6 & Pyhton Consortium & https://www.python.org/; RRID:SCR_008394 \\
\hline pandas 0.23 .4 & McKinney, 2010 & https://scipy.org/ \\
\hline Proteome Discoverer 2.2 & ThermoFisher Scientific & Cat\#OPTON-30795; RRID:SCR_014477 \\
\hline MaxQuant 1.6 & Cox and Mann, 2008 & https://www.maxquant.org/; RRID:SCR_014485 \\
\hline Perseus 1.6.2.3 & Tyanova et al., 2016 & https://www.maxquant.org/; RRID:SCR_015753 \\
\hline Numpy 1.15 .4 & van der Walt et al., 2011 & https://scipy.org/; RRID:SCR_008633 \\
\hline Cytoscape 3.5 .1 & Shannon et al., 2003 & https://cytoscape.org/; RRID:SCR_003032 \\
\hline BiNGO 3.0.3 & Maere et al., 2005 & $\begin{array}{l}\text { https://www.psb.ugent.be/cbd/papers/BiNGO/ } \\
\text { Home.html; RRID:SCR_005736 }\end{array}$ \\
\hline EnrichmentMap 3.1.0 & Merico et al., 2010 & $\begin{array}{l}\text { http://apps.cytoscape.org/apps/ } \\
\text { enrichmentmap; RRID:SCR_016052 }\end{array}$ \\
\hline Origin Pro 2018 & OriginLab & $\begin{array}{l}\text { https://www.originlab.com/2018; } \\
\text { RRID:SCR_014212 }\end{array}$ \\
\hline ReactomeFI 6.1.0 & Wu and Haw, 2017 & http://apps.cytoscape.org/apps/reactomefiplugin \\
\hline matplotlib 3.0.1 & Hunter, 2007 & https://scipy.org/; RRID:SCR_008624 \\
\hline scikit-learn 0.20 .1 & Pedregosa et al., 2011 & $\begin{array}{l}\text { https://scikit-learn.org/stable/index.html; } \\
\text { RRID:SCR_002577 }\end{array}$ \\
\hline
\end{tabular}

e1 Molecular Cell 77, 913-925.e1-e4, February 20, 2020 


\begin{tabular}{lll}
\hline Continued & & \\
\hline REAGENT or RESOURCE & SOURCE & IDENTIFIER \\
\hline Other & & \\
\hline QExactive HF Orbitrap MS & Thermo Fisher Scientific & Cat\#IQLAAEGAAPFALGMBFZ \\
Orbitrap Fusion Lumos Tribrid MS & Thermo Fisher Scientific & Cat\#IQLAAEGAAPFADBMBHQ \\
\hline
\end{tabular}

\section{LEAD CONTACT AND MATERIALS AVAILABILITY}

Further information and requests for resources and reagents should be directed to and will be fulfilled by the Lead Contact, Christian Münch (ch.muench@em.uni-frankfurt.de).

\section{EXPERIMENTAL MODEL AND SUBJECT DETAILS}

Cell lines and culture conditions

$\mathrm{HeLa}$ (human epithelial cervix-adenocarcinoma, female) cells were cultured in a humidified growth chamber at $37^{\circ} \mathrm{C}$ with $5 \% \mathrm{CO}_{2}$ with RPMI1640 medium (GIBCO, 21875034) containing 10\% FBS (GIBCO, 10270-106). To obtain fully labeled samples, cells were shifted to RPMI1640 medium for SILAC (GIBCO, 88365) containing $100 \mu \mathrm{g} / \mathrm{mL}$ Arg10 (Cambridge Isotope Laboratories), $100 \mu \mathrm{g} / \mathrm{mL}$ Lys 8 (Cambridge Isotope Laboratories), 10\% FBS and cultured for two weeks to ensure full label incorporation until cells were harvested.

For pulse labeling experiments, cells were untreated or treated for $30 \mathrm{~min}$ before pulse labeling (unless stated otherwise) with the desired compound (1 $\mu \mathrm{M}$ Thapsigargin [Abcam, ab120286]; $400 \mathrm{mM} \mathrm{NaCl}$ [Sigma Aldrich]; $0.5 \mathrm{mM}$ Arsenite [Sigma Aldrich]; $500 \mathrm{nM}$ ISRIB [Sigma Aldrich, SML0843]; $1 \mu \mathrm{M}$ Torin1 [CST, 14379]; 4EGI [Selleckchem, S7369]) before washing two times with pre-warmed PBS (GIBCO) and incubation with SILAC medium containing the same concentration of the compound (where applicable) as the normal medium. Cells were grown in SILAC medium for an additional two hours (unless stated otherwise) until harvest.

\section{METHOD DETAILS}

Cell harvest and lysis

After labeling, cells were washed three times with warm PBS and lysed on the plate with lysis buffer ( $2 \%$ SDS, $50 \mathrm{mM} \mathrm{Tris-HCl} \mathrm{pH8,}$ $150 \mathrm{mM} \mathrm{NaCl}, 10 \mathrm{mM}$ TCEP, $40 \mathrm{mM}$ chloracetamide, protease inhibitor cocktail tablet [EDTA-free, Roche] and Easy-phos phosphatase inhibitor tablet [Roche]). Lysates were scraped and transferred to $2 \mathrm{~mL}$ ProteinLoBind Eppendorf tubes (Eppendorf, Z666505). Samples were incubated for $5 \mathrm{~min}$ at $95^{\circ} \mathrm{C}$ before sonication with Sonic Vibra Cell at $1 \mathrm{~s}$ ON/ $1 \mathrm{~s}$ OFF pulse for $30 \mathrm{~s}$ at a maximal amplitude of $30 \%$ to shear genomic DNA. After sonication, samples were incubated for $10 \mathrm{~min}$ at $95^{\circ} \mathrm{C}$.

Sample preparation for LC-MS ${ }^{2}$

Lysates were precipitated using three volumes of ice-cold methanol, one volume chloroform and 2.5 volumes dd $\mathrm{H}_{2} \mathrm{O}$. After centrifugation at $14,000 \mathrm{~g}$ for $45 \mathrm{~min}$ at $4^{\circ} \mathrm{C}$, the upper aqueous phase was aspirated and three volumes of ice-cold methanol added. Samples were mixed and proteins pelleted by centrifugation at $14,000 \mathrm{~g}$ for $5 \mathrm{~min}$ at $4^{\circ} \mathrm{C}$. Supernatant was discarded and pellets washed one additional time with ice-cold methanol. Protein pellets were dried at room temperature for further use. Proteins were resuspended in $8 \mathrm{M}$ Urea, $10 \mathrm{mM}$ EPPS pH8.2, and $1 \mathrm{mM} \mathrm{CaCl}$ and protein concentration determined using a $\mu \mathrm{BCA}$ assay (ThermoFisher Scientific, 23235). Samples were then diluted to $2 \mathrm{M}$ urea using digestion buffer (10 mM EPPS $\mathrm{pH} 8.2,1 \mathrm{mM} \mathrm{CaCl}_{2}$ ) and incubated with LysC (Wako Chemicals) at 1:50 (w/w) ratio overnight at $37^{\circ} \mathrm{C}$. The next day digestion reactions were further diluted to $1 \mathrm{M}$ Urea using digestion buffer and incubated at a 1:100 $(\mathrm{w} / \mathrm{w})$ ratio of Trypsin (Promega, V5113) for an additional $6 \mathrm{~h}$ at $37^{\circ} \mathrm{C}$. Digests were acidified using trifluoroaceticacid (TFA) to a pH of 2-3 and peptides purified using SepPak C18 columns (Waters, WAT054955) according to the manufacturer's protocol. Eluates were dried and stored for further processing.

Peptides were resuspended in TMT-labeling buffer (0.2 M EPPS pH8.2, 10\% Acetonitrile) and peptide concentration determined by $\mu B C A$. Peptides were mixed with TMT reagents (ThermoFisher Scientific, 90111, A37724, 90061) in 1:2 (w/w) ratio $(2 \mu \mathrm{g}$ TMT reagent per $1 \mu \mathrm{g}$ peptide). Reactions were incubated for one hour at RT and subsequently quenched by addition of hydroxylamine to a final concentration of $0.5 \%$ at RT for $15 \mathrm{~min}$. Samples were pooled in equimolar ratio (unless stated otherwise), acidified, and dried for further processing.

Before MS-analysis, peptide samples were purified using Empore C18 (Octadecyl) resin material (3M Empore). Material was activated by incubation with Methanol for $5 \mathrm{~min}$, followed by one wash each with $70 \%$ acetonitrile/ $0.1 \%$ TFA and $5 \%$ acetonitrile/ $0.1 \%$ TFA. Samples were resuspended in $5 \%$ acetonitrile, $0.1 \%$ TFA and loaded to resin material. Peptides were washed with 
$5 \%$ acetonitrile/0.1\% TFA and eluted with $70 \%$ acetonitrile (ACN). Samples were dried and resuspended in $0.1 \%$ formic acid (FA) for $\mathrm{LC}-\mathrm{MS}^{2 / 3}$.

High-pH Reverse Phase fractionation

Peptides were either fractionated using a Dionex Ultimate 3000 analytical HPLC or a High pH Reversed phase fractionation kit (ThermoFisher Scientific). The latter was used according to manufacturer's instructions.

For high $\mathrm{pH}$ reversed phase fractionation on the Dionex HPLC, $500 \mathrm{\mu g}$ of pooled and purified TMT-labeled samples were resuspended in $10 \mathrm{mM}$ ammonium-bicarbonate (ABC), $5 \%$ ACN, and separated on a $250 \mathrm{~mm}$ long C18 column (Aeris Peptide XB-C18, $4.6 \mathrm{~mm}$ ID, $2.6 \mu \mathrm{m}$ particle size; Phenomenex) using a multistep gradient from 100\% Solvent A (5\% ACN, $10 \mathrm{mM} \mathrm{ABC}$ in water) to $60 \%$ Solvent B $(90 \%$ ACN, $10 \mathrm{mM} \mathrm{ABC}$ in water) over $70 \mathrm{~min}$. Eluting peptides were collected every $45 \mathrm{~s}$ into a total of 96 fractions, which were cross-concatenated into 24 fractions and dried for further processing.

\section{Mass spectrometry}

Unless stated otherwise, peptides were resuspended in 0.1\% FA and separated on an Easy nLC 1200 (ThermoFisher Scientific) and a $22 \mathrm{~cm}$ long, $75 \mu \mathrm{m}$ ID fused-silica column, which had been packed in house with $1.9 \mu \mathrm{m}$ C18 particles (ReproSil-Pur, Dr. Maisch), and kept at $45^{\circ} \mathrm{C}$ using an integrated column oven (Sonation). Peptides were eluted by a non-linear gradient from $5 \%-38 \%$ acetonitrile over 120 min and directly sprayed into a QExactive HF mass spectrometer equipped with a nanoFlex ion source (ThermoFisher Scientific) at a spray voltage of $2.3 \mathrm{kV}$. Full scan MS spectra (350-1400 m/z) were acquired at a resolution of 120,000 at m/z 200, a maximum injection time of $100 \mathrm{~ms}$ and an AGC target value of $3 \times 10^{6}$. Up to 20 most intense peptides per full scan were isolated using a 1 Th window and fragmented using higher energy collisional dissociation (normalized collision energy of 35). MS/MS spectra were acquired with a resolution of 45,000 at $\mathrm{m} / \mathrm{z} 200$, a maximum injection time of $80 \mathrm{~ms}$ and an AGC target value of $1 \times 10^{5}$. Ions with charge states of 1 and $>6$ as well as ions with unassigned charge states were not considered for fragmentation. Dynamic exclusion was set to $20 \mathrm{~s}$ to minimize repeated sequencing of already acquired precursors.

Unfractionated test samples were separated on an Easy nLC II (ThermoFisher Scientific) and a $15 \mathrm{~cm}$ long, $75 \mu \mathrm{m}$ ID fused-silica column, which has been packed in house with $3 \mu \mathrm{m}$ C18 particles (ReproSil-Pur, Dr. Maisch), and kept at $45^{\circ} \mathrm{C}$ using an integrated column oven (Sonation). Peptides were eluted by a non-linear gradient from 5\%-35\% acetonitrile over 125 min and directly sprayed into a LTQ Orbitrap Elite mass-spectrometer equipped with a nanoFlex ion source (ThermoFisher Scientific) at a spray voltage of 2.3 kV. Full scan MS spectra $(350-1650 \mathrm{~m} / \mathrm{z}$ ) were acquired at a resolution of 120,000 at $\mathrm{m} / \mathrm{z} 200$, a maximum injection time of $100 \mathrm{~ms}$ and an AGC target value of $1 \times 10^{6}$ charges. Up to 20 most intense peptides per full scan were isolated in the ion-trap using a 2 Th window and fragmented using higher energy collisional dissociation (normalized collision energy of 35). MS/MS spectra were acquired with a resolution of 60,000 at $\mathrm{m} / \mathrm{z} 200$, a maximum injection time of $200 \mathrm{~ms}$ and an AGC target value of $5 \times 10^{4}$. Ions with charge states of one as well as ions with unassigned charge states were not considered for fragmentation. Dynamic exclusion was set to $60 \mathrm{~s}$ to minimize repeated sequencing of already acquired precursors.

For $\mathrm{MS}^{2}$ and $\mathrm{MS}^{3}$ comparison, samples were shot on a Fusion Lumos Mass Spectrometer (Thermo Fisher Scientific). Peptides were resuspended in 0.1\% FA and separated on an Easy $\mathrm{nLC} 1200$ (ThermoFisher Scientific) and a $22 \mathrm{~cm}$ long, $75 \mu \mathrm{m}$ ID fused-silica column, which has been packed in house with $1.9 \mu \mathrm{m} \mathrm{C18}$ particles (ReproSil-Pur, Dr. Maisch), and kept at $45^{\circ} \mathrm{C}$ using an integrated column oven (Sonation). Peptides were eluted by a non-linear gradient from $5 \%-38 \%$ acetonitrile over 120 min and directly sprayed into a Fusion Lumos mass spectrometer equipped with a nanoFlex ion source (ThermoFisher Scientific) at a spray voltage of $2.6 \mathrm{kV}$. Full scan MS spectra (350-1400 m/z) were acquired at a resolution of 120,000 at m/z 200, a maximum injection time of $100 \mathrm{~ms}$ and an AGC target value of $1 \times 10^{6}$ charges. Up to 15 most intense peptides per full scan were isolated using a 1 Th window and fragmented using higher energy collisional dissociation (normalized collision energy of 38 ). $\mathrm{MS}^{2}$ spectra were acquired with a resolution of 50,000 at $\mathrm{m} / \mathrm{z} 200$, a maximum injection time of $110 \mathrm{~ms}$ and an AGC target value of $5 \times 10^{4}$. Ions with charge states of 1 and $>6$ as well as ions with unassigned charge states were not considered for fragmentation. Dynamic exclusion was set to $45 \mathrm{~s}$ to minimize repeated sequencing of already acquired precursors.

For $\mathrm{MS}^{3}$ measurements, $\mathrm{MS}^{2}$ scans were performed in the lonTrap (Turbo) with an isolation window of 0.4 Th, a maximum injection time of $120 \mathrm{~ms}$ and CID fragmented using a collision energy of $35 \%$ for $10 \mathrm{~ms}$. SPS-MS ${ }^{3}$ was performed on the 10 most intense MS ${ }^{2}$ fragment ions with an isolation window of $\left.0.7 \mathrm{Th}_{\left(M S^{1}\right.}\right)$ and $2 \mathrm{~m} / \mathrm{z}\left(\mathrm{MS}^{2}\right)$. lons were fragmented using $\mathrm{HCD}$ with a normalized collision energy of 60 and analyzed in the Orbitrap with a resolution setting of 50,000 at $\mathrm{m} / \mathrm{z} 200$, scan range of $100-1000 \mathrm{~m} / \mathrm{z}$, AGC target value of $1.5 \times 10^{5}$ and a maximum injection time of $150 \mathrm{~ms}$.

\section{Western Blotting}

Protein samples were separated by SDS-PAGE under reducing conditions. Proteins were transferred to $0.45 \mu \mathrm{M}$ nitrocellulose membranes and probed with primary antibodies. Primary antibodies were used in 5\% BSA in PBS in stated dilution (ACTB [SantaCruz] 1:5,000, EIF4EBP1 total [CST] 1:50,000, p-EIF2S1 [S51 Abcam] 1:2,000) for one hour at room temperature. Secondary antibodies (IRDye 680RD Donkey anti-mouse [Li-Cor], IRDye 800CW Donkey anti-rabbit [Li-Cor]) were used in 1:20,000 dilution in PBS and incubated for $30 \mathrm{~min}$ in the dark. Membranes were washed and imaged using an Odyssey CLx imager (Li-Cor). 


\section{QUANTIFICATION AND STATISTICAL ANALYSIS}

Processing of raw files

Raw files were analyzed using Proteome Discoverer (PD) 2.2 software (ThermoFisher Scientific). Files were recalibrated using the Homo sapiens SwissProt database (TaxID:9606, version 2017-06-07) with methionine oxidation (+15.995) as dynamic modification and carbamidomethyl (Cys,+57.021464), TMT6 (N-terminal, +229.1629) and TMT6 $(+229.1629)$ at lysines as fixed modifications. Spectra were selected using default settings and database searches performed using SequestHT node in PD. Database searches were performed against trypsin digested Homo sapiens SwissProt database and FASTA files of common contaminants ('contaminants.fasta' provided with MaxQuant) for quality control. Fixed modifications were set as TMT6 at the N terminus and carbamidomethyl at cysteine residues. As dynamic modifications TMT6 (K), TMT6+K8 $(\mathrm{K},+237.177)$, Arg10 $(\mathrm{R},+10.008)$ and methionine oxidation were set. After search, posterior error probabilities were calculated and PSMs filtered using Percolator using default settings. Consensus Workflow for reporter ion quantification was performed with default settings, except the minimal signal-to-noise ratio was set to 5 . Results were then exported to Excel files for further processing.

For SILAC only samples, raw files were analyzed using MaxQuant 1.6 (Cox and Mann, 2008), with default settings using the Homo sapiens SwissProt database (TaxID:9606, version 2017-06-07)

Data Analysis and statistics

Excel files were used as input for a custom made in-house Python pipeline. Python 3.6 was used together with the following packages: pandas 0.23.4 (McKinney, 2010), numpy 1.15.4 (van der Walt et al., 2011), matplotlib 3.0.1 (Hunter, 2007). Excel files with peptide data were read in and each channel was normalized to the lowest channel based on total intensity. For each peptide sequence, all possible modification states containing a heavy label were extracted and the intensities for each channel were averaged between all modified peptides. Baseline subtraction was performed by subtracting the measured intensities for the non-SILAC-labeled sample from all other values. Negative intensities were treated as zero. For relative quantification, channel values were divided by the abundance in the booster channel. The heavy label incorporation at the protein level was calculated by taking the median of all peptide sequences belonging to one unique protein accession. These values were combined with the standard protein output of PD 2.2 to add annotation data to the master protein accessions.

Log2 fold changes were calculated by log2 transformation of the ratio between the mean of the replicates of treated samples versus the control samples. Significance was assessed by unpaired, two-sided Student's $t$ test. $P$ values were adjusted by Benjamini-Hochberg FDR correction. Adjusted $P$ values lower than 0.05 were considered as significant. N represents number of independent replicates. Error bars, unless stated otherwise, indicate the standard deviation of replicates. Unless stated otherwise significance was defined as adjusted $P$ values $<0.05$. Adjusted $P$ value and fold change cutoffs were applied as indicated. For clustering and enrichment analyses (see below) q value cutoffs of 0.001 were used for significance definition.

Plotting and fitting of data was performed with Origin Pro 2018. For linear regression $P$ values were calculated with Origin and raw $P$ values used for statistics.

Multidimensional scaling

MDS was performed with Python 3.6 with scikit-learn 0.20.1 (Pedregosa et al., 2011) and pandas 0.23.4. Samples were standardized by removing the mean and scaled to unit variance. Resulting Z scores were subjected to MDS analysis with default settings. Dimensions were plotted using Origin Pro 2018 software.

Hierarchical clustering

Hierarchical cluster analysis for all samples was performed using Perseus (Tyanova et al., 2016) software package (version 1.6.2.3) with default settings after centering and scaling of data ( $Z$ scores).

Network analysis

For network analysis, Cytoscape 3.5.1 (Shannon et al., 2003) software was used with BiNGO 3.0.3 (Maere et al., 2005) plugin for GO term analysis, EnrichmentMap 3.1.0 (Merico et al., 2010) and ReactomeFI 6.1.0 (Wu and Haw, 2017). For GO-term analyses, gene sets were extracted from data as indicated using fold change and significance cutoffs. Gene sets were analyzed using BiNGO plugin with default settings for overrepresentation with GO sets for biological processes. Enrichment files were loaded into EnrichmentMap plugin for filtering. $Q$ value cutoff was set to 0.001 as default and edge similarity cutoff was adjusted to 0.6 .

For analysis on individual protein level, gene sets were analyzed using ReactomeFl. Gene sets were then Fl annotated, clustered and modules were analyzed for Reactome pathway enrichment with a q-value cutoff of 0.001 . Clusters were then manually annotated using the most prominent enriched pathways.

\section{DATA AND CODE AVAILABILITY}

The mass spectrometry proteomics data have been deposited to the ProteomeXchange Consortium via the PRIDE (Perez-Rivero et al., 2019) partner repository with the dataset identifiers PXD015438 and PXD014377. 
Instrument Logic Increases Identifications during Multiplexed Translatome Measurements 


\section{analytical}

\section{Instrument Logic Increases Identifications during Mutliplexed Translatome Measurements}

Kevin Klann and Christian Münch*

Cite This: Anal. Chem. 2020, 92, 8041-8045

Read Online

ACCESS | Lill Metrics \& More | 回 Article Recommendations | (s Supporting Information

ABSTRACT: Pulsed Stable Isotope Labeling in Cell culture (SILAC) approaches allow measurement of protein dynamics, including protein translation and degradation. However, its use for quantifying acute changes has been limited due to low labeled peptide stoichiometry. Here, we describe the use of instrument logic to select peaks of interest via targeted mass differences (TMD) for overcoming this limitation. Comparing peptides artificially mixed at low heavy-to-light stoichiometry measured using standard data dependent acquisition with or without TMD revealed 2-3-fold increases in identification without significant loss in quantification precision for both $\mathrm{MS}^{2}$ and $\mathrm{MS}^{3}$ methods. Our benchmarked method approach increased throughput by reducing the necessary machine time. We anticipate that all pulsed SILAC measurements, combined with tandem mass tagging (TMT) or not, would greatly benefit from instrument logic based approaches.

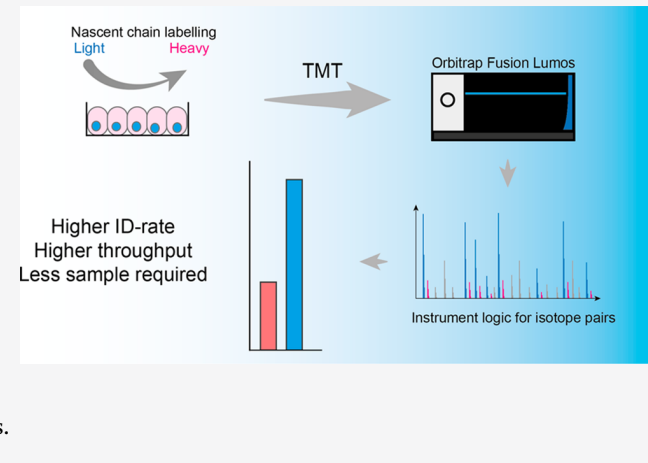
1 ost protein dynamics measurements by mass spectromLabeling in Cell culture (SILAC) or unnatural amino acid analogues. ${ }^{1-3}$ Combining these labeling approaches with tandem mass tagging (TMT) enables highly reproducible multiplexed measurements. ${ }^{4-9}$ However, a major challenge when using pulsed SILAC (pSILAC) approaches to study protein dynamics in a time-resolved manner is the low stoichiometry of labeled peptides, preventing their identification and quantification. We recently described multiplexed enhanced PROtein Dynamics (mePROD) proteomics that use a booster channel (peptides derived from fully SILAC labeled cells) to specifically increase the signal of labeled peptides only, to allow their quantification. ${ }^{10}$ However, labeled peptides remain at low stoichiometry compared to their nonlabeled counterparts, resulting in only a small fraction of mass spectrometer measurement time to be used to measure labeled peptides.

The new generation of Orbitrap mass spectrometers, such as the Orbitrap Fusion, Orbitrap Fusion Lumos, Orbitrap Eclipse, and Exploris480, offer the possibility to use instrument logic workflows, which we reasoned could potentially overcome this problem to a large extent. These filters enable on-the-fly identification of label pairs in the survey scan, such as that produced by SILAC, and subsequent targeting of one or both ions for $\mathrm{MS}^{2}$. We hypothesized that low stoichiometry SILAC samples would greatly benefit from targeted mass difference (TMD) based instrument methods. A similar rationale has previously been used for targeted experiments ${ }^{11}$ but, to our knowledge, not for data dependent acquisition (DDA) measurements.

Here, we assessed whether TMD can improve identification rates of TMT-multiplexed pSILAC samples. We found that label identification increased to approximately 2.5 -fold in an unfractionated sample, without influencing quantification accuracy.

\section{EXPERIMENTAL SECTION}

Cell Culture. HeLa cells were cultured as described previously. ${ }^{10}$

Sample Preparation. Lysates for MS sample preparation were obtained and TMT-SILAC samples prepared as previously described. ${ }^{10}$

Liquid Chromatography Mass Spectrometry. Samples were prepared for mass spectrometry analysis as described previously. ${ }^{10}$ After sample preparation, $1 \mu \mathrm{g}$ of peptide was resuspended in $2 \%$ acetonitrile (ACN) $/ 1 \%$ formic acid (FA) and separated on an Easy nLCII (Thermo Fisher Scientific) using a $25 \mathrm{~cm}$ long, $75 \mu \mathrm{M}$ inner diameter fused-silica column packed in house with $3 \mu \mathrm{M}$ C18 particles (ReproSil-Pur, Dr.

Received: April 23, 2020 Accepted: May 22, 2020

Published: May 22, 2020 


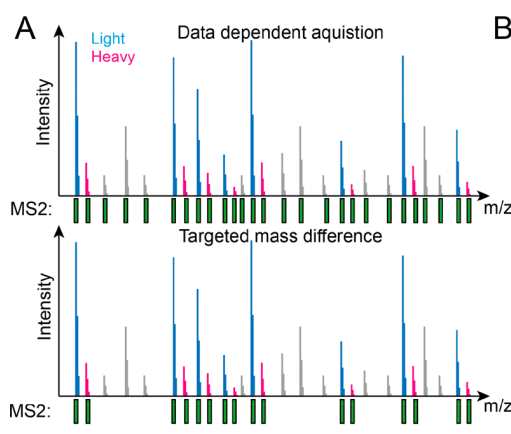

B

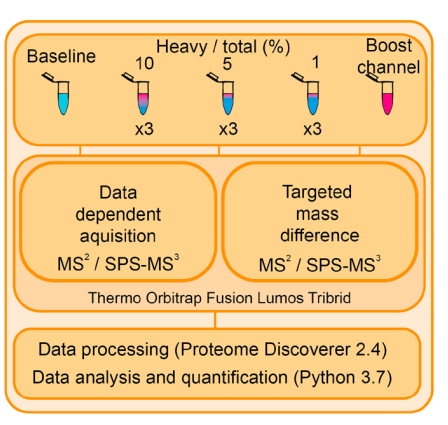

E

D Lysine containing peptides

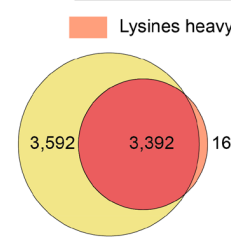

TMD

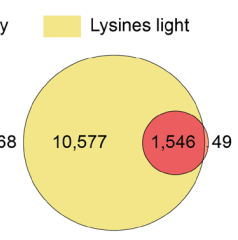

DDA

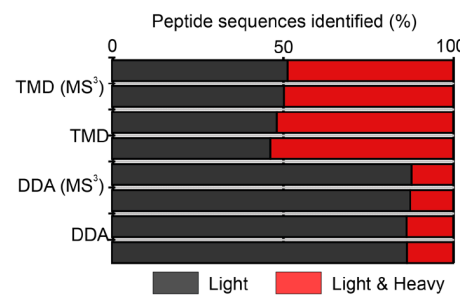

C

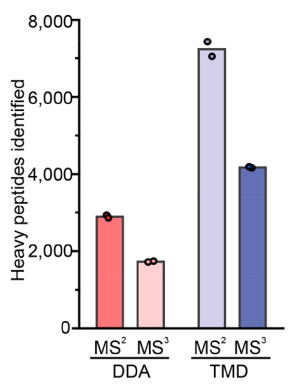

G
$\mathrm{F}$

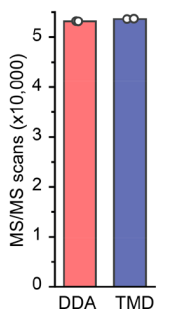

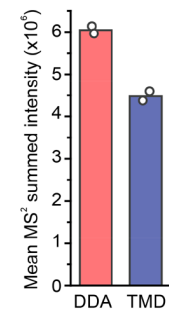

Figure 1. Instrument logic measurements increase identification and quantification rate in pulsed SILAC-TMT experiments. (A) Scheme of instrument logic based methods. Isotope pairs are identified online and subsequent scans only performed on identified pairs. (B) Experimental scheme, low stoichiometry SILAC ratios were mixed and combined with baseline and boost channel, labeled with TMT11 and measured either by data dependent acquisition (DDA) or targeted mass difference (TMD) with $\mathrm{MS}^{2}$ or $\mathrm{MS}^{3}$ acquisition settings. Data processing was performed using Proteome Discoverer 2.4 and Python 3.7. (C) Number of identified heavy peptides for all tested methods $(n=2)$. Bar represents mean. (D) Comparison between heavy and light lysines identified. (E) Percentage of heavy light distribution over all replicates and methods. (F) Number of MS/MS scans performed during same gradient time by DDA and TMD $(n=2)$. (G) Mean MS ${ }^{2}$ summed intensity for DDA and TMD based methods $(n=2)$. Bars represent mean of replicates.

Maisch) and kept at $45{ }^{\circ} \mathrm{C}$. Peptides were eluted by a nonlinear gradient from 4 to $40 \% \mathrm{ACN}$ over $120 \mathrm{~min}$ and sprayed into an Orbitrap Fusion Lumos mass spectrometer. The exact settings for each of the examined methods can be found in the Supporting Information.

Data Analysis. Raw files were analyzed using Proteome Discoverer (PD) 2.4 (ThermoFisher Scientific). Spectra were selected using default settings and database searches performed using the SequestHT node in PD. Database searches were carried out with databases of trypsin digested proteomes (Homo sapiens SwissProt database [TaxID:9606, 2017-06-07/ 2018-11-21]) and FASTA files of common contaminants ("contaminants.fasta" provided together with MaxQuant) for quality control. Fixed modifications were set as TMT6 at the $\mathrm{N}$-terminus and carbamidomethyl at cysteine residues. As dynamic modifications, TMT6 (K), TMT6+K8 $(\mathrm{K},+237.177)$, $\operatorname{Arg} 10(\mathrm{R},+10.008)$, and methionine oxidation were set. After a search, posterior error probabilities were calculated and perfect spectrum matches (PSMs) were filtered using Percolator using default settings. Consensus Workflow for reporter ion quantification was performed with default settings, except the minimal signal-to-noise ratio was set to 5. Results were then exported to Excel files for further processing. Excel files were used as input for a custom-made in-house Python pipeline. Python 3.6 was used together with the following packages: pandas $0.23 .4,{ }^{12}$ numpy $1.15 .4,{ }^{13}$ and scipy 1.3.0., as described previously. ${ }^{10}$ For injection time normalization, summed TMT intensities per PSM were divided by the injection time for that PSM, scaled by a factor of 1000, and split on the individual channels by their original ratios.

Isolation interference was calculated by summing the intensities for all TMT channels per PSM and dividing the baseline value by the sum. Assuming isolation interference to be stochastically even across all channels, the resulting value was multiplied by the number of channels used.

\section{RESULTS AND DISCUSSION}

During mePROD or comparable experimental measurements, the signal of interest (e.g., the heavy peptide, representing newly synthesized proteins) usually ranges up to around $10 \%$ of the corresponding light peptide intensity. During DDA, the top $N$ most intense ions are selected for the dependent $\operatorname{scan}(\mathrm{s})$, which are most likely nonlabeled peptides, due to their inherently higher intensity. Thus, we hypothesized that identifying the isotope pair in the survey scan and targeting both ions of the pair for $\mathrm{MS}^{2}$ or $\mathrm{MS}^{3}$, respectively, would increase the amount of measured signal of interest while enabling normalization on total protein level (Figure 1A).

To assess whether instrument logic based workflows using TMD targeting are beneficial for pSILAC-TMT methods, we constructed a mePROD experiment with artificially mixed, substoichiometric heavy peptide ratios (Figure 1B). The sample contained a mix of peptides derived from lysates of nonlabeled or heavy (SILAC) labeled HeLa extracts at heavy/ 
A

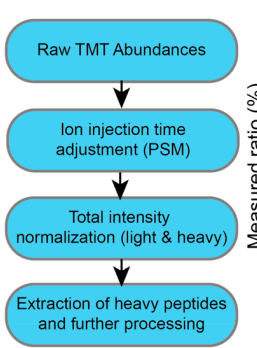

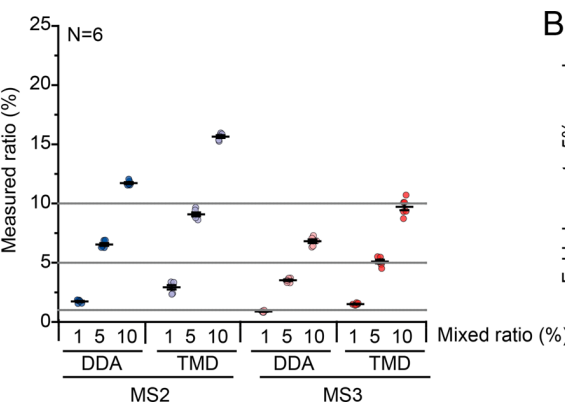

$\mathrm{B}$

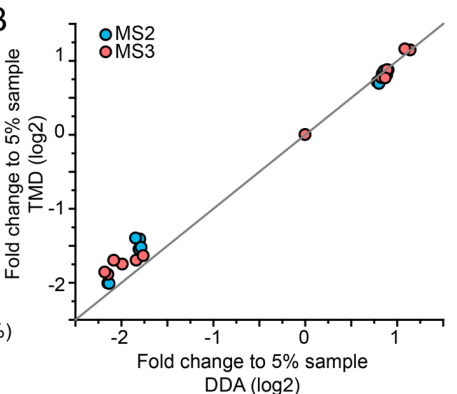

Figure 2. Absolute and relative quantification accuracy of TMD measurements. (A) Scheme of normalization approach used for determination of heavy/total ratio. Median measured ratio of all heavy PSMs compared to fully labeled booster $(n=6$, over two multiplexes) for DDA and TMD measurements. Gray lines indicate mixed ratios, black lines indicate mean of replicates. (B) Comparison of dynamic range of $\mathrm{MS}^{2}$ and $\mathrm{MS}^{3}$ based relative quantification to reference samples $(5 \%$ mixed ratio) for TMD and DDA approaches. Gray line indicates reference diagonal.

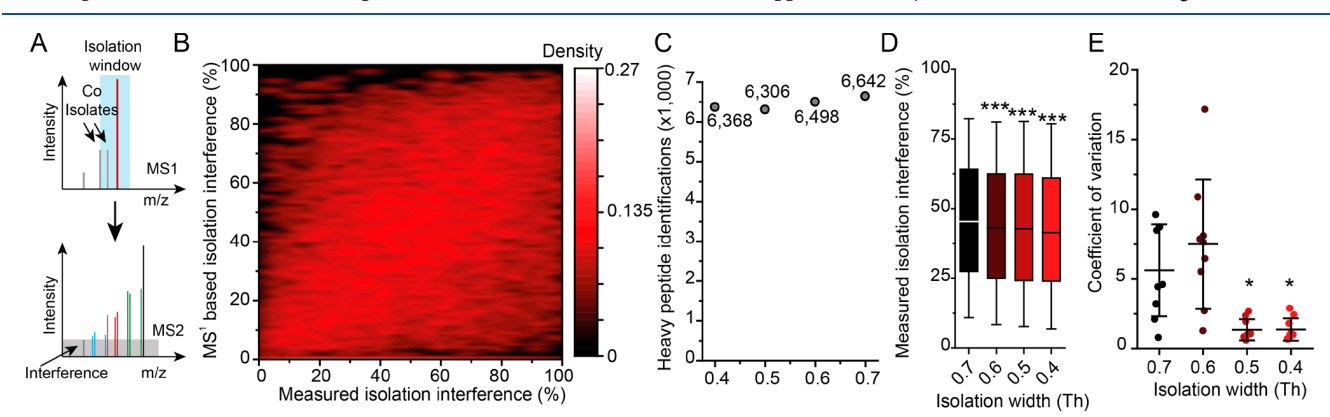

Figure 3. Narrower isolation window reduces isolation interference and variation while maintaining identification rate. (A) Scheme of experimental determination of coisolation for each PSM. Light-only baseline channel abundance represents coisolated light peptides in heavy PSM. Noise subtraction can overcome ratio compression. (B) Density plot for measured isolation interference and isolation interference predicted from survey scan (PD 2.4). (C) Heavy peptide identifications by TMD in dependency of isolation width. (D) Measured interference in dependency of different isolation windows. $* * * P<0.001$. Boxes represent $25-75 \%$ quantiles, and whiskers indicate SD. (E) Coefficient of variation of measured heavy/ total ratios with different isolation windows. ${ }^{*} P<0.05(n=9$, one multiplex). Middle bars represent median, and error bars indicate SD.

total ratios between 1 and $10 \%$ in triplicate. In addition, we included a TMT-labeled booster channel and a nonlabeled noise channel for a mePROD measurement setup that allows heavy peptide signal amplification and noise determination, respectively. ${ }^{10}$ We observed a 2.5 -fold increase in identified heavy peptides from the same sample when comparing TMD to standard DDA measurements, irrespective of acquisition by $\mathrm{MS}^{2}$ or $\mathrm{MS}^{3}$ methods (Figure 1C). The majority of measured heavy peptide sequences was also identified in their nonisotopic form (Figure 1D). TMD improved the percentage of isotope labeled peptides identified relative to total by about 3 fold to about $50 \%$ in $\mathrm{MS}^{2}$ and $\mathrm{MS}^{3}$ measurements (Figure 1E). The total number of scans performed by the spectrometer remained the same (Figure $1 \mathrm{~F}$ ). We observed a drop in mean $\mathrm{MS}^{2}$ intensities consistent with a higher percentage of scans carried out for low abundant peptides (Figure 1G).

Next, we examined the accuracy of heavy peptide quantification by comparing the signal of all channels after data analysis to the booster channel. Total intensity normalization of the full data set (light and heavy) resulted in an overestimation of the measured heavy to total ratios in both $\mathrm{MS}^{2}$ and $\mathrm{MS}^{3}$ (Figure S1). We expected this to be the result of the normalization procedure, rather than the measured data. Due to the constrained nature of the population of ions measured in a TMT experiment, ${ }^{14}$ the extracted intensities of the reporter ions are not faithfully reflecting the peptide intensity. For MS/MS scans, a fixed amount of ions is collected, independent of the precursor abundance, thus creating technical constraint for reporter ion intensities that can be reached. As a result, normalization by summing TMT intensities during SILAC hyperplexing might distort the calculated results. We therefore included an additional calculation in the data analysis: measured abundances were corrected for the injection time, which was needed to reach the AGC-target value, as an experimentally determined value approximating ion abundance (Figure 2A). Subsequently, corrected values were normalized and processed as before. This additional correction step reduced ratio distortion observed in $\mathrm{MS}^{2}$ and $\mathrm{MS}^{3} \mathrm{TMD}$ measurements, with only minor effects on DDA-based quantifications. While TMD-MS ${ }^{2}$ measurements still showed a 1.5 -fold overestimation of the heavy to total ratio, TMD-MS ${ }^{3}$ exhibited the most accurate quantification over the whole range of ratios tested. When assessing relative fold changes instead of absolute values (i.e., heavy/total ratios), DDA and TMD methods performed equally well, irrespective of $\mathrm{MS}^{2}$ or $\mathrm{MS}^{3}$ measurements used (Figure 2B). In most experimental setups, only relative quantification is required. For these, we recommend using 
$\mathrm{MS}^{2}$ methods as these outperform $\mathrm{MS}^{3}$ in the number of identifications.

A common problem during $\mathrm{MS}^{2}$ and (to a lesser extent) $\mathrm{MS}^{3}$ based quantification is the isolation of interfering peptides distorting quantification results. Precursor ion contamination in $\mathrm{MS}^{2}$ results in ratio compression and was overcome by multinotch $\mathrm{MS}^{3}$ approaches. ${ }^{15}$ We recently showed that the inclusion of a baseline channel for background subtraction in mePROD measurements is sufficient to overcome ratio compression. ${ }^{10}$ Strikingly, the baseline channel in mePROD experiments provides with an experimental system to measure isolation interference by determining isolation interference from highly abundant light peptides (Figure 3A). Notably, we observed that the measured isolation interference did not correlate with the isolation interference predicted from survey scans for filtering of perfect spectrum matches (PSMs) (Figures $3 \mathrm{~B}$ and $\mathrm{S} 2 \mathrm{~A} / \mathrm{B}$ ). This was irrespective of $\mathrm{MS}^{2}$ or $\mathrm{MS}^{3}$ methods being used (Figure S2A/B). Using an $\mathrm{MS}^{3}$ method reduced the measured isolation interference while still observing a broad range of interference values among heavy PSMs. TMD approaches in general exhibited a higher isolation interference as expected due more low abundant peaks being targeted for subsequent scans (Figure S3B). We next examined whether isolation window narrowing reduces measured isolation interference. Narrowing the isolation window to 0.4 Th significantly reduced isolation interference while only resulting in a slight reduction of heavy peptide and protein identification (Figures $3 \mathrm{C} / \mathrm{D}$ and $\mathrm{S} 2 \mathrm{C}$ ). Combining postacquisition baseline correction with isolation window narrowing significantly increased accuracy with smaller variation observed (Figure 3E).

Isolation window reduction resulted in higher median $\mathrm{MS}^{2}$ fill time and decreased mean summed $\mathrm{MS}^{2}$ intensities (Figure S2D). We next asked whether increasing maximum fill times would result in an additional benefit for quantification. Increasing maximum $\mathrm{MS}^{2}$ injection time (while keeping the isolation window fixed at $0.4 \mathrm{Th}$ ) resulted in a smaller number of identified peptides as expected due to the increase in cycle times (Figure S2E). Overall, the loss in $\mathrm{MS}^{2}$ intensity was only partially rescued with median summed intensities increased by $28 \%$, while the injection time was tripled (Figure S2F) Therefore, we concluded that the reduction of the isolation window can enhance the data quality and does not need to be supplemented by higher injection times, resulting in most efficient use of the cycle time.

Taken together, we showed that instrument logic based measurements significantly increase the identification of low abundant pSILAC signals while maintaining relative quantification accuracy. This significantly reduces the machine time required and improves identifications and quantification. The benefit of TMD was particularly pronounced in conditions with large ratio differences between the used SILAC labels. In equally distributed samples, TMD increased identification and ensured measurement of both labels to calculate ratios.

We showed that experimental setups such as those in mePROD experiments allow one to experimentally assess coisolation interference in isobaric-multiplexed experiments and could provide the bases for statistical approaches to reduce ratio compression. We anticipate that instrument logic based approaches are beneficial for any pSILAC experiment (including triple SILAC labeled samples), especially when requiring high throughput or low sample input amounts.

\section{ASSOCIATED CONTENT}

\section{(S) Supporting Information}

The Supporting Information is available free of charge at https://pubs.acs.org/doi/10.1021/acs.analchem.0c01749. Instrument settings for Orbitrap Fusion Lumos for DDA and TMD based methods; overestimation of heavy to total ratios using classical total intensity normalization; effect of isolation window narrowing on the measured isolation interference in pulsed SILAC experiments (PDF)

\section{- AUTHOR INFORMATION}

\section{Corresponding Author}

Christian Münch - Institute of Biochemistry II, Faculty of

Medicine, Goethe University, 60590 Frankfurt am Main,

Germany; Frankfurt Cancer Institute, 60596 Frankfurt am Main, Germany; Cardio-Pulmonary Institute, 60590 Frankfurt am Main, Germany; @ orcid.org/0000-0003-3832-090X; Email: ch.muench@em.uni-frankfurt.de

\section{Author}

Kevin Klann - Institute of Biochemistry II, Faculty of Medicine, Goethe University, 60590 Frankfurt am Main, Germany

Complete contact information is available at:

https://pubs.acs.org/10.1021/acs.analchem.0c01749

\section{Author Contributions}

K.K performed the experiments and analyzed data. K.K. and C.M. designed experiments, analyzed the data, and wrote the manuscript. C.M. conceived and supervised the study. All authors have given approval to the final version of the manuscript.

Notes

The authors declare no competing financial interest.

The mass spectrometry proteomics data have been deposited to the ProteomeXchange Consortium via the PRIDE ${ }^{16}$ partner repository with the data set identifier PXD018445.

\section{ACKNOWLEDGMENTS}

We thank Georg Tascher for helpful comments on the manuscript and instrumentation. C.M. was supported by the European Research Council under the European Union's Seventh Framework Programme (ERC StG 803565), the Emmy Noether Program of the Deutsche Forschungsgemeinschaft (DFG, MU 4216/1-1), and the Johanna Quandt Young Academy at Goethe.

\section{REFERENCES}

(1) Schwanhäusser, B.; et al. Nature 2011, 473, 337-342.

(2) Boisvert, F.-M.; et al. Mol. Cell. Proteomics 2012, 11, M111.011429.

(3) Dieterich, D. C.; Link, A. J.; Graumann, J.; Tirrell, D. A.;

Schuman, E. M. Proc. Natl. Acad. Sci. U. S. A. 2006, 103, 9482-7.

(4) Rothenberg, D. A.; et al. iScience 2018, 9, 367-381.

(5) Savitski, M. M.; et al. Cell 2018, 173, 260-274.e25.

(6) Mathieson, T.; et al. Nat. Commun. 2018, 9, 689.

(7) Becher, I.; et al. Cell 2018, 173, 1495-1507.e18.

(8) Zecha, J.; et al. Mol. Cell. Proteomics 2018, 17, 974.

(9) Welle, K. A.; et al. Mol. Cell. Proteomics 2016, 15, 3551-3563.

(10) Klann, K.; Tascher, G.; Münch, C. Mol. Cell 2020, 77, $913-$ 925.e4.

(11) Erickson, B. K.; et al. Mol. Cell 2017, 65, 361-370.

(12) McKinney, W. Proc. 9th Python Sci. Conf. 2010, 51-56. 
(13) van der Walt, S.; Colbert, S. C.; Varoquaux, G. Comput. Sci. Eng. 2011, 13, 22-30.

(14) O’Brien, J. J.; et al. J. Proteome Res. 2018, 17, 590-599.

15) McAlister, G. C.; et al. Anal. Chem. 2014, 86, 7150.

(16) Perez-Riverol, Y.; et al. Nucleic Acids Res. 2019, 47, D442D450. 
Unbiased translation proteomics upon cell stress 


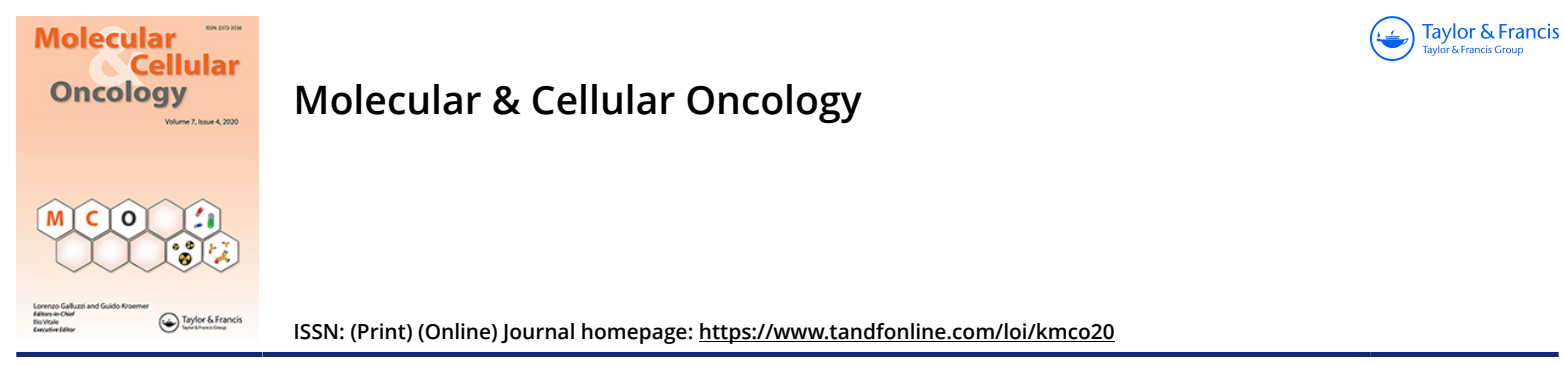

\section{Unbiased translation proteomics upon cell stress}

\section{Kevin Klann \& Christian Münch}

To cite this article: Kevin Klann \& Christian Münch (2020) Unbiased translation proteomics upon cell stress, Molecular \& Cellular Oncology, 7:4, 1763150, DOI: 10.1080/23723556.2020.1763150

To link to this article: https://doi.org/10.1080/23723556.2020.1763150

○

(c) 2020 The Author(s). Published with license by Taylor \& Francis Group, LLC.

Published online: 18 May 2020.

Submit your article to this journal $₫$

Џ Article views: 305

Q View related articles ๘

View Crossmark data $\longleftarrow$ 


\section{Unbiased translation proteomics upon cell stress}

\section{Kevin Klann $\mathbb{D}^{\mathrm{a}}$ and Christian Münch $\mathbb{D}^{\mathrm{a}, \mathrm{b}, \mathrm{c}}$}

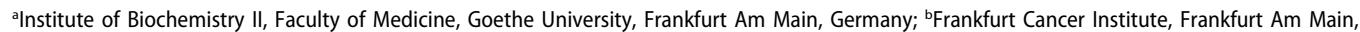
Germany; 'Cardio-Pulmonary Institute, Frankfurt Am Main, Germany

\section{ABSTRACT}

The mammalian target of rapamycin and the integrated stress response are central cellular hubs regulating translation upon stress. The precise proteins and pathway specificity of translation targets of these pathways remained largely unclear. We recently described a new method for quantitative translation proteomics and found that both pathways control translation of the same sets of proteins.

\section{ARTICLE HISTORY Received 28 March 2020 Revised 24 April 2020 Accepted 27 April 2020 \\ KEYWORDS \\ Translation proteomics, proteostasis; mTOR; ISR; integrated stress response; translatome; stress}

Measurements of protein dynamics are a useful tool to study rapid cellular changes introduced by various sorts of stimuli. Those changes are often masked in classical proteome measurements, hiding their potential for providing direct evidence of regulatory mechanisms resulting in the studied phenotype. However, protein dynamics are rarely monitored and data for most cellular stresses and modulations remains lacking. This is largely due to the limited number of methodologies available to quantify protein dynamics on a system-wide scale. In addition, many available techniques suffer from limited resolution, depth, or technical bias. Thus, new technologies and advances in proteomics approaches are needed to drive this important area of research.

Protein homeostasis is a tightly and rapidly regulated network balancing synthesis and degradation. The two central cellular hubs are the integrated stress response (ISR), causing translation attenuation and extensive remodulation of transcription upon activation, and the mammalian target of rapamycin complex 1 (mTORC1), inactivation of which inhibits translation, induces autophagy, and alters transcription. ${ }^{1,2}$ Both pathways integrate numerous stimuli emerging from a range of stresses and cell environmental conditions and ultimately result in broad remodeling of cells. Despite their central roles, the precise set of their specific translation targets had been missing. Their study is particularly difficult as the ISR and mTORC1 integrate a range of cellular stresses and conditions and are thus highly sensitive to any perturbation caused by the experimental method.

To quantify changes in the translatome, two approaches are commonly used 1) ribosome profiling (Ribo-seq), based on sequencing of mRNA pieces protected by ribosomes, 2) proteomics approaches following labeling of newly synthesized proteins to quantify translation. Both methods pose technical challenges. When monitoring conditions with strong global translation effects, such as for the ISR or mTORC1, Ribo-seq suffers from an extensive normalization bias. ${ }^{3}$ Proteomics approaches however are challenged by the low rate of newly synthesized proteins relative to the preexisting pool of (old) proteins. ${ }^{4,5}$ To overcome this issue, translation proteomics are commonly carried out using incorporation of click-reactive reagents, such as the non-natural amino acid azidohomoalanine (AHA) or puromycin, to allow biotinylation and enrichment of newly synthesized proteins before analysis. ${ }^{6}$ However, these compounds themselves cause cell stress and affect translation: 1) AHA is a methionine analogue incorporated into nascent changes through tRNA charging. To ensure high-level incorporation, cells are typically starved for methionine before addition of AHA, causing cell starvation and resulting in translation attenuation via mTORC1 inhibition. ${ }^{2}$ In addition, tRNA charging with AHA is $~ 400$ fold less efficient than with methionine. ${ }^{7}$ Uncharged tRNAs are sensed by the eukaryotic translation initiation factor 2 alpha kinase 4 (EIF2AK4, also known as GCN2), causing translation inhibition via ISR activation, ${ }^{1}$ thereby affecting the system studied. 2) Puromycin is a translation inhibitor prematurely terminating protein synthesis leading to the release of truncated proteins. Thus, puromycin labeling directly affects translation itself.

To allow monitoring translation without perturbing the system, we used pulsed stable isotope labeling of amino acids in cell culture (SILAC, i.e. heavy amino acids) labeling to mark new proteins. ${ }^{8}$ These heavy amino acids are biologically indistinguishable from their light counterpart and have no effect on cellular behavior. However, they cannot be enriched, which has largely prevented the use of pulse-SILAC labeling for measuring acute translation changes, as the intensity (amount) of newly synthesized versus old proteins is too low to be reasonably detectable by mass spectrometry with sufficient depth and accuracy. ${ }^{4,5}$ To overcome this problem, we developed multiplexed enhanced Protein Dynamics (mePROD) proteomics, combining pulsed-SILAC and tandem mass tag (TMT) multiplexing, ${ }^{9}$ with the addition of spike-ins specifically enhancing the signal of newly synthesized peptides to allow their determination. ${ }^{5}$ Overall, this equipped us with a method capable of quantifying translation of thousands of proteins in

CONTACT Christian Münch ch.muench@em.uni-frankfurt.de Institute of Biochemistry II, University Hospital Building 75, Theodor-Stern-Kai 7, 60590 Frankfurt am Main, Germany

() 2020 The Author(s). Published with license by Taylor \& Francis Group, LLC.

This is an Open Access article distributed under the terms of the Creative Commons Attribution-NonCommercial-NoDerivatives License (http://creativecommons.org/licenses/by-nc-nd/4.0/ which permits non-commercial re-use, distribution, and reproduction in any medium, provided the original work is properly cited, and is not altered, transformed, or built upon in any way. 
multiplexed experiment with up to nine samples (14 for 16-plex TMTpro), with small input requirements (e.g. <100,000 HeLa cells), and short required labeling times (i.e. minutes).

The ISR and mTORC1 both target translation of capped mRNAs. Nevertheless, the specific groups of proteins whose translation is inhibited by these pathways had been broadly assumed to be separate, despite their similar features in vivo. ${ }^{10}$ We applied mePROD translation proteomics to conditions activating the ISR or inhibiting mTORC $1 .^{5}$ Measured global translation rates reflected the extensive reduction observed by other unbiased methods. A large set of proteins showed significantly reduced relative translation rates. Strikingly, translation targets of the ISR and mTORC1 extensively overlapped, revealing that both pathways target the same translational processes (Figure 1). Surprisingly, our analyses also revealed that not pathway, but intrinsic features drive target specificity. Translation of a fraction of proteins is reduced under mild global translation inhibition, while others (mainly housekeeping proteins) are largely resistant to translation attenuation. This implies some features within the mRNA sequence, or specific proteins binding to these, that define how quickly or slowly translation of mRNAs is reduced upon stress. What these features are will be an important future question to address.

In summary, we developed a proteomics method to measure highly acute changes in translation by boosting the signal of interest. We employed this system to characterize target specificity of ISR or mTORC1-driven

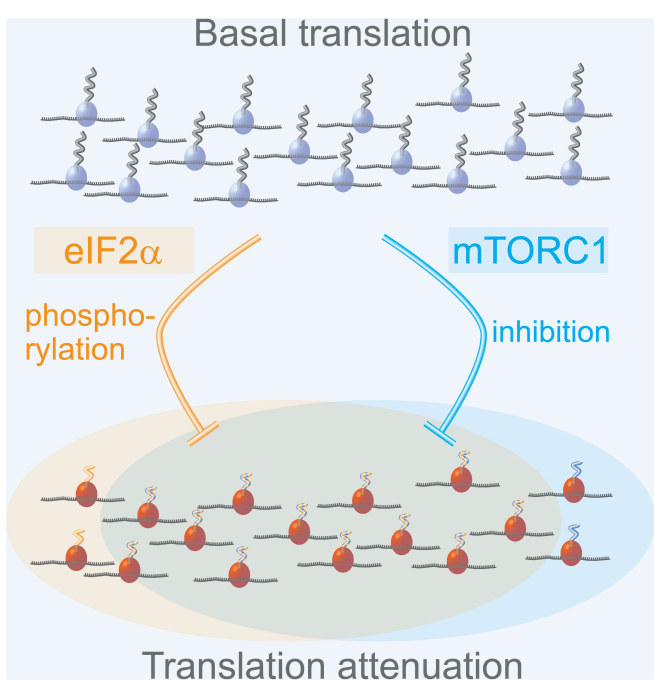

Figure 1. Overlapping control of translation by the ISR and mTORC1. Both activation of the integrated stress response (ISR), via phosphorylation of eukaryotic translation initiation factor 2 alpha (elF2a), and inhibition of mammalian target of rapamycin complex 1 (mTORC1) result in an extensive reduction in global proteins synthesis (bottom). Using multiplexed enhanced protein dynamic (mePROD) proteomics, a newly developed unbiased translatome proteomics method, revealed that the set of proteins, whose translation is controlled by the ISR or mTORC1, largely overlaps. Instead, regulation is mediated by intrinsic factors that remain unknown. translation attenuation. Our analyses revealed the sets of proteins, for which the ISR or mTORC1 inhibit translation. Strikingly, both pathways largely regulated the same set of proteins. Instead, translation rates were dependent on global protein translation inhibition. Further work will be required to determine which elements or cellular components drive this behavior and what the functional cellular consequences are.

\section{Disclosure of potential conflicts of interest}

No potential conflicts of interest were disclosed.

\section{Funding}

C.M. was supported by the European Research Council (ERC StG 803565), the Emmy Noether Program (DFG, MU 4216/1-1), and the Johanna Quandt Young Academy at Goethe.

\section{ORCID}

Kevin Klann (1) http://orcid.org/0000-0003-2276-8128 Christian Münch (D) http://orcid.org/0000-0003-3832-090X

\section{References}

1. Pakos-Zebrucka K, Koryga I, Mnich K, Ljujic M, Samali A, Gorman AM. 2016. The integrated stress response. EMBO Rep. 17:1374-1395. doi:10.15252/embr.201642195.

2. Appenzeller-Herzog C, Hall MN. 2012. Bidirectional crosstalk between endoplasmic reticulum stress and mTOR signaling. Trends Cell Biol. 22(5):274-282. doi:10.1016/j.tcb.2012.02.006.

3. Chen K, Hu Z, Xia Z, Zhao D, Li W, Tyler JK. 2015. The Overlooked Fact: fundamental Need for Spike-In Control for Virtually All Genome-Wide Analyses. Mol. Cell. Biol. 36:662-667. doi:10.1128/MCB.00970-14.

4. Bagert JD, Xie YJ, Sweredoski MJ, et al. 2014. Quantitative, Time-Resolved Proteomic Analysis by Combining Bioorthogonal Noncanonical Amino Acid Tagging and Pulsed Stable Isotope Labeling by Amino Acids in Cell Culture. Mol Cell Proteomics. 13:1352-1358. doi:10.1074/mcp.M113.031914.

5. Klann K, Tascher G, Münch C.2020. Functional Translatome Proteomics Reveal Converging and Dose-Dependent Regulation by mTORC1 and eIF2a. Mol Cell. 77(913-925.e4):913-925.e4. doi:10.1016/j.molcel.2019.11.010.

6. Dieterich DC, Link AJ, Graumann J, Tirrell DA, Schuman EM. 2006. Selective identification of newly synthesized proteins in mammalian cells using bioorthogonal noncanonical amino acid tagging (BONCAT). Proc. Natl. Acad. Sci. U. S. A. 103:9482-9487. doi:10.1073/pnas.0601637103.

7. Kiick KL, Saxon E, Tirrell DA, Bertozzi CR. 2002. Incorporation of azides into recombinant proteins for chemoselective modification by the Staudinger ligation. Proc. Natl. Acad. Sci. U. S. A. 99 (1):19-24. doi:10.1073/pnas.012583299.

8. Schwanhäusser B, Gossen M, Dittmar G, Selbach M. 2009. Global analysis of cellular protein translation by pulsed SILAC. Proteomics. 9(1):205-209. doi:10.1002/pmic.200800275.

9. Welle KA, Zhang T, Hryhorenko JR, Shen S, Qu J, Ghaemmaghami S. 2016. Time-resolved Analysis of Proteome Dynamics by Tandem Mass Tags and Stable Isotope Labeling in Cell Culture (TMT-SILAC) Hyperplexing. Mol Cell Proteomics. 15:3551-3563. doi:10.1074/mcp.M116.063230.

10. Wengrod JC, Gardner LB. 2015. Cellular adaptation to nutrient deprivation: crosstalk between the mTORC1 and eIF2 $\alpha$ signaling pathways and implications for autophagy. Cell Cycle. 14 (16):2571-2577. doi:10.1080/15384101.2015.1056947. 
Proteomics of SARS-CoV-2-infected host cells reveals therapy targets 


\title{
Article \\ Proteomics of SARS-CoV-2-infected host cells reveals therapy targets
}

\author{
https://doi.org/10.1038/s41586-020-2332-7 Denisa Bojkova ${ }^{1,7}$, Kevin Klannn ${ }^{2,7}$, Benjamin Koch ${ }^{3,7}$, Marek Widera', David Krause ${ }^{2}$, \\ Sandra Ciesek $^{1,4}$, Jindrich Cinatl $^{1 凶} \&$ Christian Münch $^{2,5,6 凶}$ \\ Accepted: 6 May 2020 \\ Published online: 14 May 2020 \\ (i) Check for updates

\begin{abstract}
A new coronavirus was recently discovered and named severe acute respiratory syndrome coronavirus 2 (SARS-CoV-2). Infection with SARS-CoV-2 in humans causes and an understanding of how SARS-CoV-2 infects cells are lacking. Here we identify the host cell pathways that are modulated by SARS-CoV-2 and show that inhibition of these pathways prevents viral replication in human cells. We established a human cell-culture model for infection with a clinical isolate of SARS-CoV-2. Using this cell-culture system, we determined the infection profile of SARS-CoV-2 by translatome ${ }^{3}$ and proteome proteomics at different times after infection. These analyses revealed that SARS-CoV-2 reshapes central cellular pathways such as translation, splicing, carbon metabolism, protein homeostasis (proteostasis) and nucleic acid metabolism. Small-molecule inhibitors that target these pathways prevented viral replication in cells. Our results reveal the cellular infection profile of SARS-CoV-2 and have enabled the identification of drugs that inhibit viral replication. We anticipate that our results will guide efforts to understand the molecular mechanisms that underlie the modulation of host cells after infection with SARS-CoV-2. Furthermore, our findings provide insights for the
\end{abstract} \\ coronavirus disease 2019 (COVID-19) and has been rapidly spreading around the globe ${ }^{1,2}$. \\ SARS-CoV-2 shows some similarities to other coronaviruses; however, treatment options \\ development of therapies for the treatment of COVID-19.
}

At the end of 2019, a cluster of cases of severe pneumonia of unknown cause was described in Wuhan (eastern China), and a SARS-like acute respiratory distress syndrome was noted in many patients. Early in January 2020, next-generation sequencing revealed that a novel coronavirus (named SARS-CoV-2) was the causal factor for the disease ${ }^{1}$, which was later designated COVID-19. SARS-CoV-2 shows high infectivity, which has resulted in rapid global spreading ${ }^{2}$.

Currently, thereisnoestablished therapyforthetreatmentofCOVID-19. Treatment is based mainly on supportive and symptomatic care ${ }^{4,5}$. Therefore, the development of therapies that inhibit infection with or replication of SARS-CoV-2 are urgently needed. Molecular examination of infected cells by unbiased proteomics approaches offers a potent strategy for revealing pathways that are relevant for viral pathogenicity to identify potential drug targets. However, this strategy depends on the availability of cell-culture models that are amenable to virus infection and sensitive proteomics approaches that can be used for temporal infection profiling in cells. SARS-CoV-2 was recently successfully isolated using the human colon epithelial carcinoma cell line Caco-2. SARS-CoV-2 replicates in gastrointestinal cells in vivo ${ }^{7}$ and is frequently detected in stool-regardless of the occurrence of diarrhoea ${ }^{8}$. Caco-2 cells were extensively used to study infection with SARS-CoV and can be used for SARS-CoV-2 infection ${ }^{6,9}$. For proteome analysis, a method-multiplexed enhanced protein dynamics (mePROD) proteomics-was recently described that enables the determination of translatome and proteome changes at high temporal resolution ${ }^{3}$. Owing to the quantification of translational changes by naturally occurring heavy isotope labelling using stable isotope labelling by amino acids in cell culture (SILAC), this method does not affect cellular behaviour and therefore enables the perturbation-free and unbiased analysis of the response of cells to viral infection.

In this study, we used quantitative translatome and proteome proteomics to obtain an unbiased profile of the cellular response to SARS-CoV-2 infection in human cells. We monitored different time points after infection and identified key determinants of the host cell response to infection. These findings revealed pathways that are relevant for SARS-CoV-2 infection. We tested several drugs that target these pathways, including translation, proteostasis, glycolysis, splicing and nucleotide synthesis pathways. These drugs inhibited SARS-CoV-2 replication at concentrations that were not toxic to the human cells, potentially providing therapeutic strategies for the treatment of COVID-19.

\section{SARS-CoV-2 rapidly replicates in cells}

To investigate potential antiviral compounds that inhibit SARS-CoV-2, we established a highly permissive SARS-CoV-2 cell-culture mode in Caco-2 cells. Addition of SARS-CoV-2 at a multiplicity of infection (MOI) of one (to enable the infection of most of the cells while prevent ing multiple infections) led to a fast progression of viral infection and visible cytopathogenic effects were apparent after $24 \mathrm{~h}$ (Fig. 1a). To

Institute of Medical Virology, University Hospital Frankfurt, Goethe University, Frankfurt am Main, Germany. ${ }^{2}$ Institute of Biochemistry II, Faculty of Medicine, Goethe University, Frankfurt am Main, Germany. ${ }^{3}$ Medical Clinic III, Nephrology, University Hospital Frankfurt, Frankfurt am Main, Germany. ${ }^{4}$ German Centre for Infection Research (DZIF), External Partner Site Frankfurt, Frankfurt am Main, Germany. ${ }^{5}$ Frankfurt Cancer Institute, Frankfurt am Main, Germany. ${ }^{6}$ Cardio-Pulmonary Institute, Frankfurt am Main, Germany. ${ }^{7}$ These authors contributed equally: Denisa

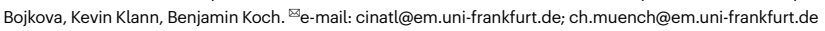




\section{Article}

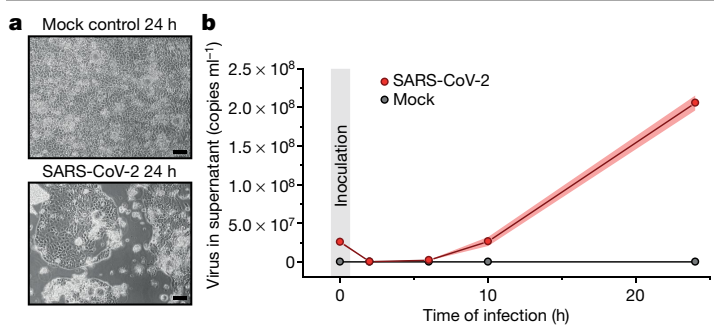

Fig. 1 | SARS-CoV-2-replication model in human cells. a, Caco-2 cells were either mock-infected or infected with SARS-CoV-2 and cultured for $24 \mathrm{~h}$. Microscopy images show the cytopathogenic effects of SARS-CoV-2 infection. Scale bars, $100 \mu \mathrm{m}$. Representative images from three independent biological replicates are shown. b, Quantitative PCR analysis of viral genome copies per ml of cell culture after the indicated infection time $(n=3$ independent biological samples). Points indicate the mean of replicate measurements and shades represent the s.d.

determine whether productive viral infection takes place in this model we measured the number of viral RNA copies in the supernatant during a 24-h time period. SARS-CoV-2 RNA molecules increased continuously after infection (Fig.1b), indicating that the virus underwent full replication cycles. Staining for viral nucleoprotein additionally revealed the production of viral proteins in most cells (Extended Data Fig. 1). Taken together, we established a functional SARS-CoV-2 cell-culture model that enables the investigation of the different steps of the life cycle of SARS-CoV-2 in cells.

\section{Translation inhibitors block replication}

To determine the temporal profile of SARS-CoV-2 infection, we infected Caco-2 cells with SARS-CoV-2, cultured them for a range of $2-24 \mathrm{~h}$ and quantified translatome and proteome changes by mePROD proteomics compared with time-matched mock-infected samples (Fig. 2a). Across all replicates, we quantified translation for 2,715 proteins and tota protein levels for 6,382 proteins (Supplementary Table 1). Principal component analysis showed that replicates clustered closely and that infected samples showed the first separation from control clusters after $6 \mathrm{~h}$ (Extended Data Fig. 2a). Many RNA viruses decrease protein synthesis in cells, as has been suggested for SARS-CoV ${ }^{10}$. When monitoring global translation rates, only minor changes in translation were observed (Fig. 2b and Extended Data Fig. 2b). We detected translation rates for five viral proteins, all of which exhibited increasing translation rates over time (Fig. 2c). To identify pathways that are potentially important for virus amplification, we determined host proteins that exhibited translation kinetics, which correlated with viral proteins. Averaged profiles of all quantified viral proteins were used as reference profiles; the distance to this profile was calculated for all quantified host proteins and a network analysis was carried out for the top $10 \%$ quantile of nearest profiles (244 proteins) (Extended Data Fig. 2c-f). Pathway analyses of the network revealed an extensive increase in the translation machinery of the host (Fig. 2d and Extended Data Fig. 2g). In addition, we detected significant enrichment of components of several other pathways, such as splicing and nucleobase synthesis (Fig. 2d).

Host translation has previously been targeted to pharmacologically inhibit the replication of diverse coronaviruses, such as SARS-CoV or MERS-CoV ${ }^{11,12}$ (Extended Data Fig. 2h). As components of the translation machinery were translated at higher rates (Fig. 2d), we hypothesized that SARS-CoV-2 replication might be sensitive to inhibition of translation. We tested two translation inhibitors-cycloheximide (an inhibitor of translation elongation) and emetine (an inhibitor of the $40 \mathrm{~S}$ ribosomal protein S14)-for their ability to reduce SARS-CoV-2 replication. Both compounds significantly inhibited SARS-CoV-2 replication at

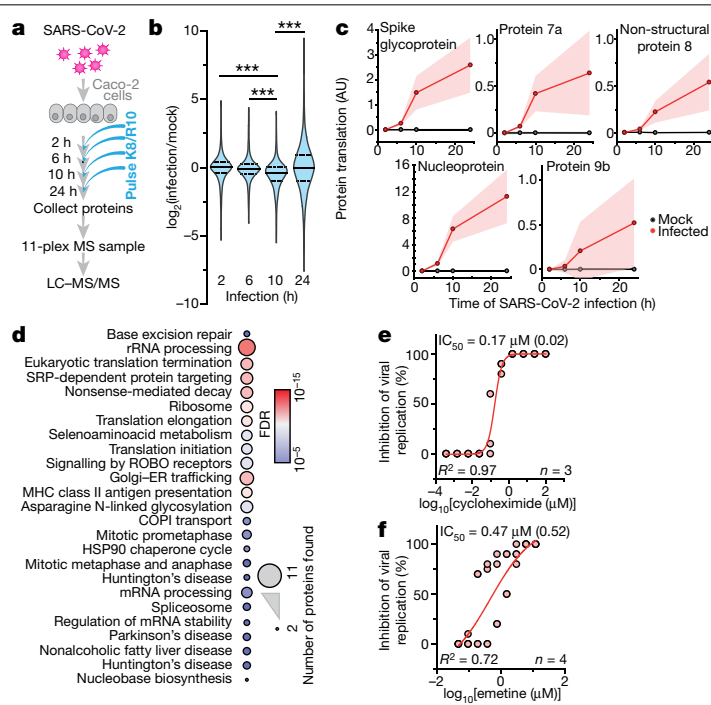

Fig. 2 Host cell translation changes after infection with SARS-CoV-2.

a, Experimental scheme for translatome and proteome measurements. Caco-2 cells were infected with SARS-CoV-2 isolated from patients, incubated as indicated and analysed by quantitative translation and whole-cell proteomics. $\mathrm{K} 8$, lysine with 8 heavy isotopes; R10, arginine with 10 heavy isotopes; LC-MS/ MS, Liquid chromatography-tandem mass spectrometry.b, Global translation rates, showed by distribution plots of mean $\log _{2}$-transformed fold changes of infected replicates to mock control for each time point and protein. The black line indicates the median and the dashed lines indicate $25 \%$ and $75 \%$ quantiles. Significance was tested by one-way ANOVA and two-sided post hoc Bonferroni test. ${ }^{* * *} P<0.001$ ( $10 \mathrm{~h}$ compared with $2 \mathrm{~h}, 4 \times 10^{-26} ; 10 \mathrm{~h}$ compared with $6 \mathrm{~h}$, $2.4 \times 10^{-23} ; 10 \mathrm{~h}$ compared with $24 \mathrm{~h}, 2.3 \times 10^{-28} ; n=2,716$ measured protein averaged from 3 independent biological samples).c. Translation of viral proteins over time. Mean translation in arbitrary units (AU; normalized and corrected summed peptide spectrum matches were averaged) is plotted for control and infected samples. Shading indicates the s.d. $(n=3)$. d, Reactome pathway analysis of top $10 \%$ proteins following viral gene expression. Pathway results are shown with the number of proteins found in the dataset and computed FDRs for pathway enrichment. ER, endoplasmic reticulum. e,f, The antiviral assays show that the inhibition of viral replication is dependent on the concentrations of cycloheximide (e, $n=3)$ and emetine $(\mathbf{f}, n=4)$. Each data poin indicates biological replicates and the red line shows the dose-response curve fit. $R^{2}$ and half-maximum inhibitory concentration $\left(\mathrm{IC}_{50}\right)$ values were computed from the curve fit and the s.d. of the $\mathrm{IC}_{50}$ is indicated in parentheses. All $n$ numbers represent independent biological samples if not stated otherwise.

concentrations that are not toxic to human Caco-2 cells (Fig. 2e, f and Extended Data Fig. 2i,j). Taken together, translatome analyses of cells infected with SARS-CoV-2 revealed the temporal profile of viral and host protein responses with prominent increases in the translation machin ery. Translation inhibitors prevented SARS-CoV-2 replication in cells.

\section{Pathways changed by SARS-CoV-2 infection}

To obtain a general understanding of changes in the host proteome after infection, we analysed system-wide differences in protein levels over time (Fig. 3a and Supplementary Table 2). Whereas early time points showed only minor changes in the host proteome, the proteome underwent extensive modulation $24 \mathrm{~h}$ after infection (Fig. 3a and Extended Data Fig. 3). Hierarchical clustering identified two main clusters of proteins that were differentially regulated. The first cluster consisted of proteins that were reduced during infection and was enriched in proteins that belonged to cholesterol metabolism (Extended Data Fig. 4a,

470 | Nature | Vol 583 | 16 July 2020 


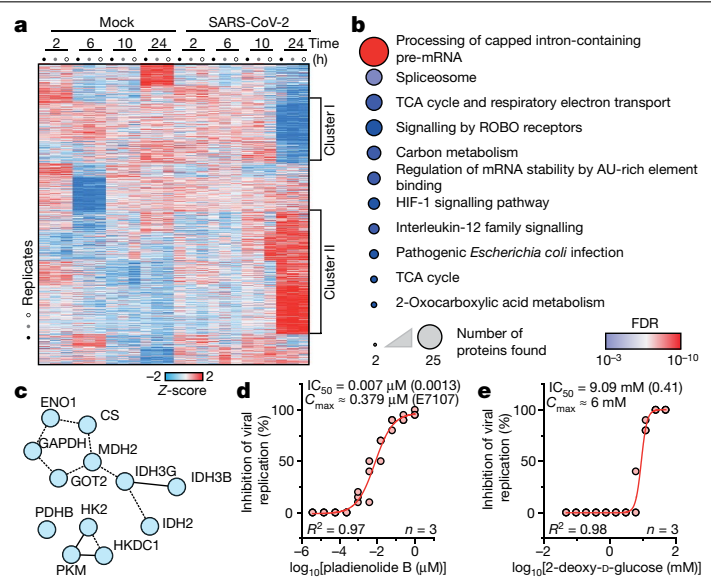

Fig. 3 |SARS-CoV-2 infection profiling reveals cellular pathways that are essential for replication. a, Patterns of protein levels across all samples. The proteins that were significantly up- or downregulated (two-sided, unpaired Student's $t$-test with equal variance assumed, $P<0.05, n=3$ ) in at least one infected sample compared with the corresponding control are shown. Data were standardized using $Z$-scoring before row-wise clustering and plotting. TCA, tricarboxylic acid.b, Reactome pathway analysis of the protein network created from cluster II, which includes host cell proteins that are increased after SARS-CoV-2 infection (a;Supplementary Table 4). Pathway results are shown with the number of proteins found in the dataset and computed FDR for pathway enrichment.c, Functional interaction network of proteins found annotated to carbon metabolism in the Reactome pathway analysis. Lines indicate functional interactions. d, e, The antiviral assays show that the inhibition of viral replication is dependent on the concentrations of pladienolide $\mathrm{B}(\mathbf{d}, n=3)$ and 2-deoxy-Dglucose $(\mathbf{e}, n=3)$. Each data point indicates a biological replicate and the red line shows the dose-response curve fit. $R^{2}$ and $\mathrm{IC}_{50}$ values were computed from the curve fit and the s.d. of $\mathrm{IC}_{50}$ is indicated in parentheses. $C_{\max }$, maximum plasma concentration; $\mathrm{E} 7107$, the indicated $C_{\mathrm{m} \text {, }}$ was obtained for the $\mathrm{E7107}$, a derivative of pladienolide B. All $n$ numbers represent independent biological samples.

b and Supplementary Table 3). The second cluster was composed of proteins that were increased by infection and revealed strong increases in RNA-modifying proteins, such as spliceosome components (consistent with translatome measurements in Fig. 2d), and carbon metabolism (Fig. 3b, c, Extended Data Fig. 5a and Supplementary Table 4). Notably, for 14 out of 25 spliceosome components that were increased after infection with SARS-CoV-2, direct binding to viral proteins of SARS-CoV or other coronaviruses had been shown ${ }^{13-16}$ (Extended Data Fig. 5b). We therefore tested whether the inhibition of splicing or glycolysis may be able to prevent SARS-CoV-2 replication. Addition of pladien olide $\mathrm{B}$, a spliceosome inhibitor that targets the splicing factor SF3B1 ${ }^{17}$ prevented viral replication at concentrations that were not toxic to the human Caco-2 cells (Fig. $3 \mathrm{~d}$ and Extended Data Fig. $5 \mathrm{c}$ ), revealing that splicing is an essential pathway for SARS-CoV-2 replication and a potential therapeutic target.

Next, we assessed the effects of the inhibition of carbon metabolism (that is, glycolysis) on SARS-CoV-2 replication. 2-deoxy-D-Glucose, an inhibitor of hexokinase (the rate-limiting enzyme in glycolysis), has previously been shown to be effective against other viruses in cell culture and suppressed infection with rhinovirus in mice ${ }^{18}$. Blocking glycolysis with non-toxic concentrations of 2-deoxy-D-glucose prevented SARS-CoV-2 replication in Caco-2 cells (Fig. 3 e and Extended Data Fig. 5d). Notably, we also observed changes in proteins that reside in the endoplasmic reticulum and that are involved in lipid metabolism (Extended Data Fig. 6), consistent with previous reports on other coronaviruse ${ }^{19}$. Together, our quantitative analyses of proteome changes
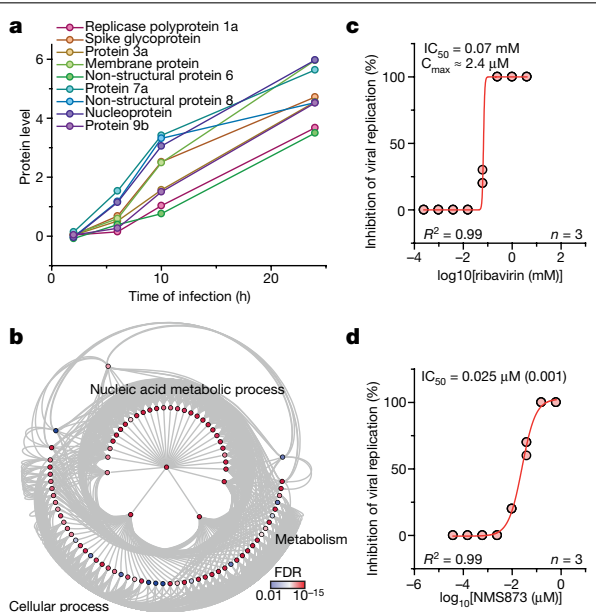

Fig. 4 Inhibition of host cell pathways induced by infection prevent SARS-CoV-2 replication. a, Protein levels of all detected viral proteins are plotted with their $\log _{2}$-transformed changes compared with the corresponding control for different infection times. Mean fold changes are plotted $(n=3)$. b, Gene ontology network analysis of host proteins that correlated with viral protein expression (FDR-adjusted $P<0.01$ ). Proteins were clustered according to the gene ontology term of the biological process and plotted as a network with FDR colour coding. Annotated pathways represent parent pathways in the network.c, d, The antiviral assays show that the inhibition of viral replication is dependent on the concentrations of ribavirin $(\mathbf{c}, n=3)$ and NMS873 $(\mathbf{d}, n=3)$. Each data point indicates a biological replicate and the red line indicates the dose-response curve fit. $R^{2}$ and $I_{50}$ values were computed from the curve fit and the s.d. of $\mathrm{IC}_{50}$ is indicated in parentheses. All $n$ numbers represent independent biological samples.

after infection with SARS-CoV-2 revealed host pathways that change after infection and revealed that spliceosome and glycolysis inhibitors are potential therapeutic agents for the treatment of COVID-19.

\section{Kinetic profiling of the infection proteome}

To identify additional potential inhibitors of SARS-CoV-2 replication, we determined proteins with abundance trajectories that were simila to the nine detected viral proteins (Fig. $4 \mathrm{a}$ and Extended Data Fig. 7a-d measurement depth does not allow us to distinguish polyprotein from processed protein). We compared the distance and false-discovery rate (FDR) for each protein to an averaged viral protein profile and performed gene ontology analysis (459 proteins with a FDR-adjusted $P<0.01$ ). We identified a major cluster of metabolic pathways, which consisted of diverse nucleic acid metabolism sub-pathways (Fig. 4b and Supplementary Table 5). Coronavirus replication depends on the availability of cellular nucleotide pools ${ }^{20}$. Compounds that interfere with nucleic acid metabolism, such as ribavirin, have been used in the past to inhibit viral replication ${ }^{21}$. We tested the effect of the inhibition of nucleotide synthesis on SARS-CoV-2 replication in cells. Ribavirin, which inhibits inosine monophosphate dehydrogenase (IMPDH), the rate-limiting enzyme in de novo synthesis of guanosine nucleotides, inhibited SARS-CoV-2 replication at low micromolar and clinically achievable concentrations ${ }^{22}$ (Fig. $4 \mathrm{c}$ and Extended Data Fig. 7e), consistent with data in monkey cells ${ }^{23}$. Inhibition of IMPDH had been shown to prevent replication of coronaviruses HCoV-43, CoV-NL63 and MERS-CoV but not of SARS-CoV ${ }^{24}$. Considering the clinical use of ribavirin to treat viruses such as hepatitis $\mathrm{C}$ and respiratory syncytia virus $^{25,26}$, it may be regarded as a treatment option for patients with COVID-19. 


\section{Article}

Components of the proteostasis machinery also acted in a comparable manner to the viral proteins (Extended Data Fig. 3e), consistent with the perturbation of host cell proteostasis due to the higher folding load, which is the results of the high translation rates of viral proteins. We therefore tested the effects of proteostasis perturbation on SARS-CoV-2 replication using NMS-873, a small-molecule inhibitor of the AAA ATPase p97. p97 is a key component of proteostasis, which affects protein degradation, membrane fusion, vesicular trafficking and disassembly of stress granule ${ }^{27}$. NMS- 873 has previously been shown to inhibit the replication of influenza $A$ and $B^{28}$. We show that NMS 873 inhibits SARS-CoV-2 replication at low nanomolar concentrations (Fig. 4d and Extended Data Fig. 7f). In summary, analyses of the effects of SARS-CoV-2 infection on the host cell proteome revealed major readjustments in cellular function, particularly of splicing, proteostasis and nucleotide biosynthesis. Compounds that modulate these pathways prevented SARS-CoV-2 replication in human cells.

\section{Discussion}

Identifying and testing potential drug candidates for the treatmen of COVID-19 is of high priority. So far, only limited data have been obtained that describes the response of the host cell to infection with SARS-CoV-2, preventing a databased assessment of treatment options. We describe a SARS-CoV-2 cell-infection system that can be used to determine the changes in host cell pathways after infection, which result from host cell (antiviral) responses or viral effector proteins, and assess inhibitors. At the MOI used, most of the cells were infected, enabling us to determine global changes across the whole cell population with minimal ratio compression from uninfected cells. We found that the expression of the previously described SARS-CoV-2 entry receptor ACE2 was mildly reduced after infection (Extended Data Fig. 8), consistent with a drop in ACE2 levels due to shedding by ADAM10 that has been described for SARS- $\mathrm{CoV}^{29}$. Temporal proteome and translatome proteomics showed limited translation attenuation and revealed core cellular pathways that were modulated after infection (Fig. 2). For SARS-CoV and other RNA viruses, severe effects on translation have been described ${ }^{30}$. Our observations suggest that SARS-CoV-2 reshapes host cell translation, probably by increasing the production of translation machinery components to compensate for the inhibition of host cell translation. We tested two translation inhibitors with different modes of action and found that these to efficiently prevented viral replication in cells. These findings encourage further testing of translation inhibitors for the prevention of SARS-CoV-2 replication.

Overall, our proteomics analyses highlight cellular pathways for therapeutic interventions, including a marked increase in components of the spliceosome, proteostasis and nucleotide biosynthesis pathways. This enabled us to assess new drug targets, which were based on the behaviour of SARS-CoV-2 in human cells and which had not previously been tested with other coronaviruses. Some of the inhibitors, for which we observed inhibition of SARS-CoV-2, are approved drugs, such as ribavirin, or are undergoing clinical trials (that is, 2-deoxy-D-glucose). A clinical trial for ribavirin was recently initiated (ClinicalTrials.gov; NCT04356677). Analysis of pathways that are important for viral infection in cells by combinatorial profiling using proteomics and translatomics represents a useful tool to propose likely pathways that inhibit viral replication. Determining possible compounds based on the specific cellular infection profile of the virus enables an unbiased determination of potential drug targets. Here, using such an experimental-data-driven approach, we identified several drugs that prevent SARS-CoV-2 replication in cells for further testing in clinical settings for the treatment of COVID-19.

\section{Online content}

Any methods, additional references, Nature Research reporting sum maries, source data, extended data, supplementary information, acknowledgements, peer review information; details of author contributions and competing interests; and statements of data and code availability are available at https://doi.org/10.1038/s41586-020-2332-7.

Zhu, N. et al. A novel coronavirus from patients with pneumonia in China, 2019. N. Engl. J. Med. 382, 727-733 (2020)

Zhao, S. et al. Preliminary estimation of the basic reproduction number of novel coronavirus (2019-nCoV) in China, from 2019 to 2020 : a data-driven analysis in the early phase of the outbreak. Int. J. Infect. Dis. 92, 214-217 (2020).

Klann, K., Tascher, G. \& Münch, C. Functional translatome proteomics reveal 913-925 (2020).

4. Jin. Y. . (2020).

5. She, J. et al. 2019 novel coronavirus of pneumonia in Wuhan, China: emerging attack and management strategies. Clin. Transl. Med. $\mathbf{9}, 19$ (2020).

Hoehl, S. et a Evidence of SARS-CoV-2 infection in returning travelers from Wuhan China. N. Engl. J. Med. 382, 1278-1280 (2020).

Xiao, F. et al. Evidence for gastrointestinal infection of SARS-CoV-2. Gastroenterology 158, 1831-1833(2020)

Young, B. E. et al. Epidemiologic features and clinical course of patients infected with SARS-CoV-2 in Singapore. J. Am. Med. Assoc. 323, 1488-1494 (2020).

Cinatl, J. et al. Treatment of SARS with human interferons. Lancet 362, 293-294 (2003).

10. Kamitani, W., Huang, C., Narayanan, K., Lokugamage, K. G. \& Makino, S. A two-pronged strategy to suppress host protein synthesis by SARS coronavirus Nsp1 protein. Nat. Struct. Mol. Biol. 16, 1134-1140 (2009).

11. Shen, L. et al. High-throughput screening and identification of potent broad-spectrum inhibitors of coronaviruses. J. Virol. 93, e00023-19 (2019).

12. Pillaiyar, T., Meenakshisundaram, S. \& Manickam, M. Recent discovery and development of inhibitors targeting coronaviruses. Drug Discov. Today 25, 668-688 (2020).

3. Neuman, B. W. et al. Proteomics analysis unravels the functional repertoire of coronavirus nonstructural protein 3. J. Virol. 82, 5279-5294 (2008).

14. Jourdan, S. S., Osorio, F. \& Hiscox, J. A. An interactome map of the nucleocapsid protein from a highly pathogenic North American porcine reproductive and respiratory syndrome virus strain generated using SILAC-based quantitative proteomics. Proteomics 12, 1015-1023 (2012)

15. Song, T. et al. Quantitative interactome reveals that porcine reproductive and respiratory syndrome virus nonstructural protein 2 forms a complex with viral nucleocapsid protein and cellular vimentin. J. Proteomics 142, 70-81 (2016).

16. Emmott, E. et al. The cellular interactome of the coronavirus infectious bronchitis virus nucleocapsid protein and functional implications for virus biology. J. Virol. 87 9486-9500 (2013).

17. Cretu, C. et al. Structural basis of splicing modulation by antitumor macrolide compounds. Mol. Cell 70, 265-273 (2018).

18. Gualdoni, G. A. et al. Rhinovirus induces an anabolic reprogramming in host cell metabolism essential for viral replication. Proc. Natl. Acad. Sci. USA 115, E7158-E7165 (2018).

19. Yan, B. et al. Characterization of the lipidomic profile of human coronavirus-infected cells: implications for lipid metabolism remodeling upon coronavirus replication. Viruses 11, 73 (2019)

Pruijssers, A. J. \& Denison, M. R. Nucleoside analogues for the treatment of coronavirus infections. Curr. Opin. Virol. 35, 57-62 (2019).

21. Saijo, M. et al. Inhibitory effect of mizoribine and ribavirin on the replication of severe (2005).

22. Wu, L. S. et al. Population pharmacokinetic modeling of plasma and intracellular ribavirin concentrations in patients with chronic hepatitis c virus infection. Antimicrob. Agents Chemother. 59, 2179-2188 (2015)

23. Wang, M. et al. Remdesivir and chloroquine effectively inhibit the recently emerged novel coronavirus (2019-nCoV) in vitro. Cell Res. 30, 269-271 (2020). 24. Cinatl, J. et al. Glycyrrhizin, an active component of liquorice roots, and replication of
SARS-associated coronavirus. Lancet $361,2045-2046$ (2003).

Martin, P. \& Jensen, D. M. Ribavirin in the treatment of chronic hepatitis C. J. Gastroenterol. Hepatol. 23, 844-855 (2008).

26. Marcelin, J. R., Wilson, J. W. \& Razonable, R. R. Oral ribavirin therapy for respiratory syncytial virus infections in moderately to severely immunocompromised patients. Transpl. Infect. Dis. 16, 242-250 (2014).

27. Ye, Y., Tang, W. K., Zhang, T. \& Xia, D. A mighty "protein extractor" of the cell: structure and function of the p97/CDC48 ATPase. Front. Mol. Biosci. 4, 39 (2017).

28. Zhang, J. et al. Identification of NMS-873, an allosteric and specific p97 inhibitor, as a broad antiviral against both influenza A and B viruses. Eur. J. Pharm. Sci. 133, 86-94 (2019)

29. Jia, H. P. et al. Ectodomain shedding of angiotensin converting enzyme 2 in human airway epithelia. Am. J. Physiol. Lung Cell. Mol. Physiol. 297, L84-L96 (2009).

30. Narayanan, K. et al. Severe acute respiratory syndrome coronavirus nsp1 suppresses host gene expression, including that of type linterferon, in infected cells. J. Virol. 82, 4471-4479 (2008)

Publisher's note Springer Nature remains neutral with regard to jurisdictional claims in published maps and institutional affiliations.

(c) The Author(s), under exclusive licence to Springer Nature Limited 2020 


\section{Methods}

Cell culture

Human Caco-2 cells, derived from colon carcinoma, were obtained from the Deutsche Sammlung von Mikroorganismen und Zellkulturen (DSMZ; AC169). The cell-authentification certificate from DSMZ is available and cells have been tested negative for mycoplasma infection.

Cells were grown at $37^{\circ} \mathrm{C}$ in minimal essential medium (MEM) supplemented with $10 \%$ fetalbovineserum(FBS) and containing100IU $/ \mathrm{ml}$ penicillin and $100 \mu \mathrm{g} / \mathrm{ml}$ streptomycin. All culture reagents were purchased from Sigma.

\section{Virus preparation}

SARS-CoV-2 was isolated from samples of travellers returning from Wuhan (China) to Frankfurt (Germany) using the human colon carcinoma cell line Caco-2 as described previously ${ }^{6}$. SARS-CoV-2 stocks used in the experiments had undergone one passage on Caco- 2 cells and were stored at $-80^{\circ} \mathrm{C}$. Virus titres were determined as $\mathrm{TCID}_{50} / \mathrm{ml}$ in confluent cells in 96-well microtitre plates.

\section{Quantification of viral RNA}

SARS-CoV-2 RNA from cell-culture supernatant samples was isolated using AVL buffer and the QIAamp Viral RNA Kit (Qiagen) according to the manufacturer's instructions. Absorbance-based quantification of the RNA yield was performed using the Genesys 1OS UV-Vis Spectrophotometer (ThermoScientific). RNA was subjected to OneStep qRT-PCR analysis using the Luna Universal One-Step RT-qPCR Kit (New England Biolabs) and a CFX96 Real-Time System, C1000 Touch Thermal Cycler. Primers were adapted from the WHO protocol ${ }^{31}$ targeting the open-reading frame for RNA-dependent RNA polymerase (RdRp): RdRP_SARSr-F2 (GTGARATGGTCATGTGTGGCGG) and RdRP_SARSr-R1 (CARATGTTAAASACACTATTAGCATA) using $0.4 \mu \mathrm{M}$ per reaction. Standard curves were created using plasmid DNA (pEX-A128-RdRP) that contained the corresponding amplicon regions of the $R d R P$ target sequence according to GenBank accession number NC_045512. For each condition three biological replicates were used. Mean \pm s.d. were calculated for each group.

\section{Antiviral and cell viability assays}

Confluent layers of Caco-2 cells in 96-well plates were infected with SARS-CoV-2 at a MOI of 0.01 . Virus was added together with drugs and incubated in MEM supplemented with $2 \%$ FBS with different drug dilutions. Cytopathogenic effects were assessed visually $48 \mathrm{~h}$ after infection. To assess the effects of drugs on Caco-2 cell viability, confluent cell layers were treated with different drug concentration in 96-well plates. The viability was measured using the Rotitest Vital (Roth) according to the manufacturer's instructions. Data for each condition were collected for at least three biological replicates. For dose-response curves, data were fitted with all replicates using OriginPro 2020 with the following equation:

$$
y=A 1+\frac{A 2-A 1}{1+10^{\left(\log x_{0}-x\right) p}}
$$

$\mathrm{IC}_{50}$ values were generated by Origin together with metrics for curve fits

Detection of the nucleoprotein of SARS-CoV-2

Viral infection was assessed by staining of SARS-CoV-2 nucleoprotein In brief, cells were fixed with acetone:methanol $(40: 60)$ solution and immunostaining was performed using a monoclonal antibody directed against the nucleoprotein of SARS-CoV-2 (1:500, Sinobiological, 40143-R019-100ul), which was detected with a peroxidase-conjugated anti-rabbit secondary antibody (1:1,000, Dianova), followed by addition of AEC substrate.
Isotope labelling and cell lysis

In brief, $2 \mathrm{~h}$ before collection, cells were washed twice with warm PBS to remove interfering medium and cultured for an additional $2 \mathrm{~h}$ with DMEM medium containing $84 \mathrm{mg} / \mathrm{l} \mathrm{L}$-arginine $\left({ }^{13} \mathrm{C} 6{ }^{15} \mathrm{~N} 4\right.$ (R10); Cambridge Isotope Laboratories, CNLM-539-H) and $146 \mathrm{mg} / \mathrm{l} \mathrm{L-lysine}$ $\left({ }^{13} \mathrm{C} 6{ }^{15} \mathrm{~N} 2\right.$ (K8), Cambridge Isotope Laboratories, CNLM-291-H) to labe nascent proteins. After labelling culture, the cells were washed three times with warm PBS and lysed with $95^{\circ} \mathrm{C}$ hot lysis buffer (100 mMEPPS pH 8.2, 2\% sodium deoxycholate, $1 \mathrm{mM}$ TCEP, $4 \mathrm{mM} 2$-chloracetamide protease inhibitor tablet mini EDTA-free (Roche)). Samples were then incubated for an additional $5 \mathrm{~min}$ at $95^{\circ} \mathrm{C}$, followed by sonication for $30 \mathrm{~s}$ and a further 10 -min incubation at $95^{\circ} \mathrm{C}$.

\section{Sample preparation for LC-MS/MS}

Samples were prepared as previously described ${ }^{3}$. In brief, proteins were precipitated using methanol:chloroform precipitation and resus pended in $8 \mathrm{M}$ urea and $10 \mathrm{mM}$ EPPS pH 8.2. Isolated proteins were digested with 1:50 w/w LysC (Wako Chemicals) and 1:100 w/w trypsin (Promega, Sequencing-grade) overnight at $37^{\circ} \mathrm{C}$ after dilution to a final urea concentration of $1 \mathrm{M}$. Digests were then acidified $(\mathrm{pH} 2-3)$ using TFA. Peptides were purified using C18 (50 mg) SepPak columns (Waters) as previously described. Desalted peptides were dried and $25 \mu \mathrm{g}$ of peptides were resuspended in TMT-labelling buffer $(200 \mathrm{mM}$ EPPS pH 8.2,10\% acetonitrile). Peptides were subjected to TMT labelling with 1:2 peptide TMT ratio (w/w) for $1 \mathrm{~h}$ at room temperature. The labelling reaction was quenched by addition of hydroxylamine to a final concentration of $0.5 \%$ and incubation at room temperature for an additional $15 \mathrm{~min}$. Labelled peptides were pooled and subjected to high $\mathrm{pH}$ reverse $\mathrm{Phase}$ fractionation with the $\mathrm{HpH}$ RP Fractionation kit (ThermoFisher Scientific) following the manufacturer's instructions. All multiplex reactions were mixed with a bridge channel, which consists of a control sample labelled in one reaction and split to all multiplexed reactions in equimolar amounts.

\section{LC-MS/MS}

Peptides were resuspended in $0.1 \%$ formic acid and separated on an Easy nLC 1200 (ThermoFisher Scientific) and a 22-cm-long 75- $\mu \mathrm{m}$-inner-diameter fused-silica column, which had been packed in house with 1.9- $\mu \mathrm{m} \mathrm{C18}$ particles (ReproSil-Pur, Dr. Maisch), and kept at $45^{\circ} \mathrm{C}$ using an integrated column oven (Sonation). Peptides were eluted by a nonlinear gradient from 5-38\% acetonitrile over $120 \mathrm{~min}$ and directly sprayed into a QExactive HF mass spectrometer equipped with a nanoFlex ion source (ThermoFisher Scientific) at a spray voltage of $2.3 \mathrm{kV}$. Full-scan MS spectra $(350-1,400 \mathrm{~m} / z)$ were acquired at a resolution of 120,000 at $\mathrm{m} / z 200$, a maximum injection time of $100 \mathrm{~ms}$ and an AGC target value of $3 \times 10^{6}$. Up to 20 most intense peptides per full scan were isolated using a $1 \mathrm{Th}$ window and fragmented using higher-energy collisional dissociation (normalized collision energy of 35). MS/MS spectra were acquired with a resolution of 45,000 at $\mathrm{m} / z 200$, a maximum injection time of $80 \mathrm{~ms}$ and an AGC target value of $1 \times 10^{5}$. Ions with charge states of 1 and $>6$ as well as ions with unassigned charge states were not considered for fragmentation. Dynamic exclusion was set to 20 s to minimize repeated sequencing of already acquired precursors.

\section{LC-MS/MS data analysis}

Raw files were analysed using Proteome Discoverer 2.4 software (ThermoFisher Scientific). Spectra were selected using default settings and database searches performed using SequestHT node in Proteome Discoverer. Database searches were performed against a trypsin-digested Homo sapiens SwissProt database, the SARS-CoV-2 database (Uniprot pre-release) and FASTA files of common contaminants ('contaminants.fasta' provided with MaxQuant) for quality control. Fixed modifications were set as TMT6 at the $\mathrm{N}$ terminus and carbamidomethyl at cysteine residues. One search node was 


\section{Article}

set up to search with TMT6 (K) and methionine oxidation as static modifications to search for light peptides and one search node was set up with TMT6+K8 $(K,+237.177), \operatorname{Arg} 10(\mathrm{R},+10.008)$ and methionine oxidation as static modifications to identify heavy peptides. Searches were performed using Sequest HT. After each search, posterior error probabilities were calculated and peptide spectrum matches (PSMs) filtered using Percolator using default settings. Consensus Workflow for reporter ion quantification was performed with default settings, except the minimal signal-to-noise ratio was set to 5 . Results were then exported to Excel files for further processing. For proteome quantification all PSMs were summed intensity normalized, followed by IRS ${ }^{32}$ and $\mathrm{TMM}^{33}$ normalization and peptides corresponding to a given UniProt accession were summed, including all modification states.

For translatome measurements, Excel files were processed in Python, as previously described ${ }^{3}$.Python 3.6 was used together with the following packages: pandas $0.23 .4^{34}$, numpy $1.15 .4^{35}$ and scipy 1.3 .0 . Excel files with normalized PSM data were read in and each channel was normalized to the lowest channel based on total intensity. For each peptide sequence, all possible modification states containing a heavy label were extracted and the intensities for each channel were averaged between all modified peptides. Baseline subtraction was performed by subtracting the measured intensities for the non-SILAC-labelled sample from all other values. Negative intensities were treated as zero. The heavy label incorporation at the protein level was calculated by summing the intensities of all peptide sequences belonging to one unique protein accession. These values were combined with the standard protein output of Proteome Discoverer 2.4 to add annotation data to the master protein accessions.

\section{Hierarchical clustering and profile comparison}

Hierarchical cluster analysis and comparison with viral protein profiles for all samples was performed using Perseus ${ }^{36}$ software package (version 1.6.5.0) after centring and scaling of data ( $Z$-scores). $K$-means pre-processing was performed with a cluster number of 12 and a maximum of 10 iterations. For the comparison of profiles, the viral profiles were $Z$-scored and averaged to generate reference profile. Profiles of all proteins were compared to the reference (Pearson), distances and FDRs were computed.

Network analysis

For network analysis, Cytoscape $3.7 .1^{37}$ software was used with the BiNGO3.0.338 plugin for gene ontology analysis, EnrichmentMap 3.1.0 and ReactomeFI 6.1.0 ${ }^{40}$. For gene ontology analyses, gene sets were extracted from data as indicated using fold change and significance cut-offs.

\section{Statistical analysis}

No statistical methods were used to predetermine sample size. Signifi cance was, unless stated otherwise, tested using unpaired two-sided Student's $t$-tests with equal variance assumed. Statistical analysis was performed using OriginPro 2020 analysis software. For network and gene ontology analysis all statistical computations were performed by the corresponding packages.

\section{Reporting summary}

Further information on research design is available in the Nature Research Reporting Summary linked to this paper.

\section{Data availability}

The LC-MS/MS proteomics data have been deposited in the Pro teomeXchange Consortium via the PRIDE $^{41}$ partner repository with the dataset identifier PXD017710. We furthermore created a webpage (http://corona.papers.biochem2.com/), in which the presented data is visualized for easy access of the published data.

Corman, V. M. et al. Detection of 2019 novel coronavirus (2019-nCoV) by real-time RT-PCR. Euro Surveill. 25, 2000045 (2020).

32. Plubell, D. L. et al. Extended multiplexing of tandem mass tags (TMT) labeling reveals age and high fat diet specific proteome changes in mouse epididymal adipose tissue. Mol. Cell. Proteomics 16, 873-890 (2017)

33. Robinson, M. D. \& Oshlack, A. A scaling normalization method for differential expression analysis of RNA-seq data. Genome Biol. 11, R25 (2010).

34. McKinney, W. Data structures for statistical computing in Python. In Proc. 9th Python in Science Conference 56-61 (SCIPY, 2010).

35. van der Walt, S., Colbert, S. C. \& Varoquaux, G. The NumPy Array: a structure for efficient numerical computation. Comput. Sci. Eng. 13, 22-30 (2011).

Tyanova, S. et al. The Perseus computational platform for comprehensive analysis of (prote)omics data. Nat. Methods 13, 731-740 (2016).

Shannon, P. et al. Cytoscape: a software environment for integrated models of

biomolecular interaction networks. Genome Res. 13, 2498-2504 (2003).

8. Maere, S., Heymans, K. \& Kuiper, M. BiNGO: a Cytoscape plugin to assess overrepresentation of gene ontology categories in biological networks. Bioinformatics 21 3448-3449 (2005)

39. Merico, D., Isserlin, R., Stueker, O., Emili, A. \& Bader, G. D. Enrichment map: a network-based method for gene-set enrichment visualization and interpretation. PLOS ONE 5, e13984 (2010). 40. Wu, G. \& Haw, R. Functional interaction network construction and analysis for disease discovery. Methods Mol. Biol. 1558, 235-253 (2017).

41. Perez-Riverol, Y. et al. The PRIDE database and related tools and resources in 2019 improving support for quantification data. Nucleic Acids Res. 47, D442-D450 (2019).

42. Chen, J.-Y. et al. Interaction between SARS-CoV helicase and a multifunctional cellular protein (Ddx5) revealed by yeast and mammalian cell two-hybrid systems. Arch. Virol. 154, 507-512 (2009)

43. Wong, H.-H. et al. Genome-wide screen reveals valosin-containing protein requirement for coronavirus exit from endosomes. J. Virol. 89, 11116-11128 (2015).

Acknowledgements We thank C. Pallas and L. Stegmann for experimental support and G. Tascher and M. Adrian-Allgood for proteomics support. J.C. acknowledges funding by the C. C.M. Was supported by the European Research Council under the European Union's Seventh Framework Programme (ERCStG 803565), CRC 1177 and the Emmy Noether Programme of the at Goethe and an Aventis Foundation Bridge Award.

Author contributions B.K., J.C. and C.M. conceived the study. D.B. carried out tissue-culture Aurk, virus experiments and cytotoxicity assays. K.K. performed proteomic a Blyses of vira infection kinetics and bioinformatics analyses. M.W. carried out quantitative PCRs. B.K. analysed literature for established inhibitors in viral therapy. D.K. developed the online too for data visualization. S.C. J.C. and C.M. supervised the work. K.K. J.C. and C.M. wrote the initial manuscript, with contributions from all authors. All authors read and approved the final manuscript.

Competing interests The authors declare no competing interests.

Additional information Supple 2332 .

Correspondence and requests for materials should be addressed to J.C. or C.M. Peer review information Nature thanks Ileana M. Cristea, Michael P. Weekes and the other, reviewer(s) for their contribution to the peer review of this work. Reprints and permissions information is available at http://www.nature com/reprints. 
Growth factor receptor signaling inhibition prevents SARS-CoV-2 replication 


\section{Journal Pre-proof}

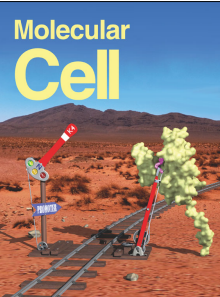

Jindrich Cinatl

PII:

S1097-2765(20)30549-9

DOI: https://doi.org/10.1016/j.molcel.2020.08.006

Reference: MOLCEL 7615

To appear in:

Molecular Cell

Received Date: 1 May 2020

Revised Date: 8 June 2020

Accepted Date: 7 August 2020

Please cite this article as: Klann, K., Bojkova, D., Tascher, G., Ciesek, S., Münch, C., Cinatl, J., Growth factor receptor signaling inhibition prevents SARS-CoV-2 replication, Molecular Cell (2020), doi: https:// doi.org/10.1016/j.molcel.2020.08.006.

This is a PDF file of an article that has undergone enhancements after acceptance, such as the addition of a cover page and metadata, and formatting for readability, but it is not yet the definitive version of record. This version will undergo additional copyediting, typesetting and review before it is published in its final form, but we are providing this version to give early visibility of the article. Please note that, during the production process, errors may be discovered which could affect the content, and all legal disclaimers that apply to the journal pertain.

(C) 2020 Published by Elsevier Inc. 


\section{Growth factor receptor signaling inhibition prevents SARS- CoV-2 replication}

Kevin Klann ${ }^{1,{ }^{*}}$, Denisa Bojkova ${ }^{2,{ }^{*},}$, Georg Tascher ${ }^{1}$, Sandra Ciesek $^{2,3}$, Christian Münch ${ }^{1,4,5,+}$, Jindrich Cinat ${ }^{2,+}$

5 Affiliations:

${ }^{1}$ Institute of Biochemistry II, Faculty of Medicine, Goethe University, Frankfurt am Main, Germany

${ }^{2}$ Institute of Medical Virology, University Hospital Frankfurt, Frankfurt am Main, Germany

${ }^{3}$ German Centre for Infection Research (DZIF), External partner site Frankfurt, Germany

$10{ }^{4}$ Frankfurt Cancer Institute, Frankfurt am Main, Germany; Cardio-Pulmonary Institute, Frankfurt am Main, Germany

${ }^{5}$ Lead author

*These authors contributed equally

+These authors contributed equally. Corresponding author. Email: ch.muench@em.unifrankfurt.de (C.M.) and cinatl@em.uni-frankfurt.de (J.C.)

Keywords: SARS-CoV-2, Proteomics, Signaling, TMT, Phosphoproteomics, COVID-19

\section{Summary}

SARS-CoV-2 infections are rapidly spreading around the globe. The rapid development of therapies is of major importance. However, our lack of understanding of the molecular

20 processes and host cell signaling events underlying SARS-CoV-2 infection hinder therapy development. We employed a SARS-CoV-2 infection system in permissible human cells to study signaling changes by phospho-proteomics. We identified viral protein phosphorylation and defined phosphorylation-driven host cell signaling changes upon infection. Growth factor receptor (GFR) signaling and downstream pathways were

25 activated. Drug-protein network analyses revealed GFR signaling as key pathway targetable by approved drugs. Inhibition of GFR downstream signaling by five compounds prevented SARS-CoV-2 replication in cells, assessed by cytopathic effect, viral dsRNA production, and viral RNA release into the supernatant. This study describes host cell signaling events upon SARS-CoV-2 infection and reveals GFR signaling as

30 central pathway essential for SARS-CoV-2 replication. It provides with novel strategies for COVID-19 treatment.

\section{Introduction}

Severe acute respiratory syndrome coronavirus 2 (SARS-CoV-2), a novel coronavirus, has been rapidly spreading around the globe since the beginning of 2020. In people, it causes coronavirus

35 disease 2019 (COVID-19) often accompanied by severe respiratory syndrome (Chen et al., 2020). To conquer the global health crisis triggered by COVID-19, rapidly establishing drugs is required to dampen the disease course and relieve healthcare institutions. Thus, repurposing of already available and (ideally) approved drugs might be essential to rapidly treat COVID- 19 . Many studies for proposing repurposing of specific drugs have been conducted in the last

40 months, but mostly remain computational without tests in infection models (Smith and Smith, 2020; Wang, 2020). In addition, they are hindered by the lack of knowledge about the molecular mechanisms of SARS-CoV-2 infection and the resulting host-cell responses required to allow 
viral replication. To rationally repurpose drugs, a molecular understanding of the infection and the changes within the host cell pathways is essential. Experimentally identifying viral targets in 45 the cell allows candidate drugs to be selected with high confidence for further testing in the clinics to reduce the risks for patients resulting from tests with drugs lacking in vitro validation.

Growth factor receptor (GFR) signaling plays important roles in cancer pathogenesis and has also been reported to be crucial for infection with some viruses (Beerli et al., 2019; Kung et al., 2011; Zhu et al., 2009). GFR activation leads to the modulation of a wide range of cellular

50 processes, including proliferation, adhesion, or differentiation (Yarden, 2001). Various viruses, such as Epstein-Barr virus, influenza, or hepatitis $C$, have been shown to use the epidermal growth factor receptor (EGFR) as an entry receptor (Eierhoff et al., 2010; Kung et al., 2011; Lupberger et al., 2011). In addition, EGFR activation can suppress interferon signaling and thus the antiviral response elicited in respiratory virus diseases, for instance influenza $A$ and

55 rhinovirus (Ueki et al., 2013). Activation of GFR signaling might play an important role also in other respiratory viruses, such as SARS-CoV-2.

In the last years, it has been shown for many viruses that modulation of host cell signaling is crucial for viral replication and might exhibit strong therapeutic potential (Beerli et al., 2019; Pleschka et al., 2001). However, how SARS-CoV-2 infection changes host cell signaling has

60 remained unclear. We recently established an in vitro cell culture model of SARS-CoV-2 infection using the colon epithelial cell line Caco-2, which is highly permissive for the virus and commonly used for the study of coronaviruses (Herzog et al., 2008; Ren et al., 2006). Here, we determine changes in the cellular phospho-protein networks upon infection with SARS-CoV-2 to gain insight into infection-induced signaling events. We found extensive rearrangements of

65 cellular signaling pathways, particularly of GFR signaling. Strikingly, inhibiting GFR signaling using prominent (anti-cancer) drugs - namely pictilisib, omipalisib, RO5126766, lonafarnib, and sorafenib - prevented SARS-CoV-2 replication in vitro, assessed by cytopathic effect and viral RNA replication and release. These compounds prevented replication at clinically achievable concentrations. Due to their clinical availability, these drugs could be rapidly transitioned towards

70 clinical trials to test their feasibility as COVID-19 treatment option.

\section{Results}

\section{Phospho-proteomics of cells infected with SARS-CoV-2}

In a previous study, we analyzed the effect of SARS-CoV-2 infection on the host cell translatome and proteome (Bojkova et al., 2020). This study found the effects 24 hours after SARS-CoV-2

75 infection especially useful for identifying druggable host pathways. To evaluate changes in intracellular signaling networks brought about by SARS-CoV-2 infection, we quantified phosphoproteome changes 24 hours after infection (Figure 1A). Caco-2 cells were mock-infected or infected with SARS-CoV-2 patient isolates (in five biological replicates at an $\mathrm{MOI}$ of 1 ) for one hour, washed, and incubated for 24 hours before cell harvest. Extracted proteins were digested

80 and split to 1) carry out whole-cell proteomics of a tandem mass tag (TMT) 10-plex samples using liquid chromatography synchronous precursor selection mass spectromety (LC-SPS-MS ${ }^{3}$ ), or 2) use Fe-NTA phosphopeptide enrichment (achieving 98\% enrichment) for phospho proteome analyses of a TMT 10-plex analyzed by LC-MS ${ }^{2}$, due to the higher precision and identification rates of $\mathrm{MS}^{2}$ based methods during phosphopeptide measurements (Hogrebe et

85 al., 2018). We identified and quantified 7,150 proteins and 16,715 different phosphopeptides for a total of 15,093 different modification sites (Figure 1B, C, S1, and Table S1, S2). The main fraction of phosphopeptides were modified serines (86.4\%), followed by threonine $(13.4 \%)$, and tyrosine (0.2\%) (Figure 1D). Upon infection, 2,197 and 799 phosphopeptides significantly increased or decreased respectively $\left(\log _{2} \mathrm{FC}>1, \mathrm{p}\right.$ value $\left.<0.05\right)$. 
90 Viral proteins are produced in the host cell and underlie (and often require) post-translational modification (PTM) by host cell enzymes (Wu et al., 2009). Accordingly, we assessed viral proteins phosphorylated in the host cell. We identified 33 modification sites on 6 different viral proteins (Figure 1E-J). Possible functions of the observed modifications largely remain unclear due to a lack of understanding of their molecular function and regulation. SARS-CoV-2 protein

95 3a was phosphorylated on the luminal side of this transmembrane protein (Figure 1E) Membrane protein $\mathrm{M}$ was phosphorylated at three serines in close proximity, at the C-terminal, cytoplasmic region of the protein (Figure $1 \mathrm{~F}$ ), suggesting a high-activity modification surface. SARS-CoV-1 protein 6 was described to accelerates infections in murine systems (Tangudu et al., 2007). We found a single phosphorylation of the SARS-CoV-2 protein homologue non-structural protein 6 in

100 host cells (Figure 1G) Protein 9b was modified at two sites (Figure 1H). However, its function in SARS-CoV-1 or SARS-CoV-2 remains unknown. Polyprotein $1 \mathrm{~b}$ is a large 7,096 amino acid protein heavily processed to generate distinct proteins in SARS-CoV-1 (Tangudu et al., 2007). We found polyprotein $1 \mathrm{~b}$ to be modified at three residues, two in a region of unknown function and one in the non-structural protein 11 (NSP11) part of the protein (Figure 1I). Our data cannot distinguish whether phosphorylation occurred before or after cleavage and whether phosphorylation may affect processing. SARS-CoV-2 nucleoprotein was heavily phosphorylated (Figure 1J). Mapping phosphosites to the structure (residues 47 to 173, PDB: 6vyo) revealed a small surface region, suggesting specific regulation and interaction changes (Figure $1 \mathrm{~K}$ ). To reveal host kinases potentially phosphorylating viral proteins, we bioinformatically assessed 110 identified phosphorylation motifs using NetPhos 3.1 and GPS5 (Blom et al., 2004; Wang et al., 2020) (Table S3). Some motifs present in nucleoprotein were predicted to be modified by CMGC kinases. Among several others, casein kinase II (CK2) kinases are part of the CMGC family and have been independently identified as interaction partners of the nucleoprotein, when expressed in cells (Gordon et al., 2020). Inhibition of CK2 kinases, could be employed to study possible

115 functional interactions between kinase and viral protein.

Taken together, we identified extensive changes in phosphorylation of host and viral proteins after SARS-CoV-2 infection. The roles of viral protein modifications remain unclear. However, targeting the corresponding host kinases may offer new treatment strategies.

\section{Signaling pathways modulated upon infection}

120 To identify the key host signaling pathway networks modulated by infection, we carried out protein-protein co-regulation analysis on all proteins quantified in phosphorylation and total protein level. We first standardized phosphorylation and total protein levels by individual Zscoring to compare the different datasets. Subsequently, to merge phosphorylation and proteome data, we collapsed all phospho-sites for each protein into one average profile and

125 calculated combined Z-scores. Patterns of co-regulation were identified using protein-protein correlation and hierarchical clustering (Figure 2A). This generalized approach allows to study large scale patterns of dependencies of protein and phosphorylation levels, that then can be dissected into individual phosphorylation sites and protein levels for downstream analysis. The dynamic landscape of the proteome revealed three main clusters of co-regulated proteins, each

130 one representing different sets of pathways (discussed in detail below):

The first cluster was mainly comprised of receptor signaling and endocytic pathways (Figure 2B). Prominent among these pathways were platelet derived growth factor receptor (PDGFR), ErbB1 (EGFR) signaling, metabolism, and various pathways associated with vesicle trafficking (Table S4). As changes in phospho-peptide abundance can represent different ratios in phosphorylated versus non-phosphorylated peptide or a change in protein abundance (with the same ratio of protein being phosphorylated), we integrated our phospho-proteome dataset with total proteome data (Figure 2C). When comparing abundances of individual phosphopeptides and their protein levels, extensive changes were observed in the phospho-proteome; however, no general 
changes were seen for the total proteome (Figure 2C, Table S2). Thus, phosphorylation changes were induced by signaling activity alteration resulting in increased phosphorylation and not due to protein abundance differences.

The second cluster was mainly comprised of proteins decreased in phosphorylation and was highly connected to cell cycle and translation initiation (Figure 2D and Table S4). We reported recently that inhibition of cellular translation prevented SARS-CoV-2 replication in cells (Bojkova

145 et al., 2020), consistent with regulation of translation by altering phosphorylation patterns. To further distinguish the regulations within this cluster, we correlated protein levels with differential phosphorylation abundance (Figure $2 \mathrm{E}$ ) and found two groups of proteins: The first was contained translation related pathways (identified in Figure $2 \mathrm{E}$ ) and was predominantly regulated by decreased modification. The second set of proteins was decreased in phosphorylation and on

150 total protein level. The majority of proteins found in the second cluster belonged to diverse cell cycle pathways. Consistent with these findings, cell cycle pathways were also enriched in the set of proteins significantly decreased on protein level (Figure 2F, S2, and Table S4 and S5). Translation pathways were not regulated on protein level to this extent.

Analysis of the third cluster revealed signaling events of the splicing machinery (Table S4)

155 possibly explaining previously observed changes in splicing machinery abundance upon SARSCoV-2 infection (Bojkova et al., 2020). Consistent with previous literature (Grimmler et al., 2005; Ilan et al., 2017; Mathew et al., 2008; Mermoud et al., 1994), we therefore hypothesized that the host splicing machinery is extensively reshaped during viral infection. This finding further supports splicing as a potential therapeutic target, in agreement with decreased SARS-CoV-2

160 pathogenic effects when inhibiting splicing by pladeinolide B. Additionally, we found carbon metabolism among the pathways showing significantly increased phosphorylation upon SARSCoV-2 infection (Table S4) in addition to previously described changes of total protein levels of enzymes part of glycolysis and carbon metabolism (Bojkova et al., 2020)(Figure S3).

Taken together, we showed that, during SARS-CoV-2 infection, specific rearrangements of 165 signaling pathways were elicited in the cellular proteome. Regulation was mainly comprised of cellular signaling and translational pathways as well as proteins regulated not only by phosphorylation, but also in total protein abundance. Proteins exhibiting decreased protein levels were significantly enriched in cell cycle proteins.

\section{Drug-target network reveals growth factor signaling as potent therapy candidate}

170 We observed over 2,000 phospho-peptides to be increased in abundance while their protein levels stay constant upon infection (Figure $2 \mathrm{C}$ and S4). This reveals differential modification activity (e.g. signaling events) for these phospho-proteins. For many kinases in cellular signaling pathways there are already approved drugs available. Hence, we investigated the potential to repurpose drugs to treat COVID-19 by mapping already available drugs via ReactomeFI to the

175 set of proteins increased in phosphorylation. We filtered the network for drugs and direct targets and found EGFR as one of the central hits, including a number of regulated proteins in the downstream signaling pathway of EGFR (Figure 3A). These downstream targets are also regulated by other GFRs and could thus also be explained by their observed activation upon SARS-CoV-2 infection (Figure 2). 28 clinically approved drugs (largely used in cancer therapy) 180 are already available to target EGFR or downstream targets. Indeed, we found a subnetwork of GFR signaling components remodeled (Figure 3B). We mapped identified members of GFR signaling and their respective phosphorylation differences upon SARS-CoV-2 infection (Figure 3C) revealing an extensive overall increase in phosphorylation of the whole pathway, including related components for cytoskeleton remodeling and receptor endocytosis. How GFR signaling might regulate SARS-CoV-2 infection is still matter for speculation. However, GFR signaling 
inhibition might provide a useful approach already implicated in SARS-CoV induced fibrosis therapy (Venkataraman and Frieman, 2017) and might be a viable strategy to treat COVID-19.

\section{Inhibition of growth factor signaling prevents viral replication}

Since GFR signaling seemed to be central during SARS-CoV-2 infection, we examined the use

190 of inhibitors as antiviral agents. Since there are several GFRs integrating their signaling and regulating a number of processes inside the cell, directly targeting downstream signaling components is likely to be more successful to prevent signaling of different GFRs and to avoid mixed effects of multiple pathways. GFR signaling, amongst others, results in activation of 1) the RAF/MEK/ERK MAPK signaling cascade and 2) integrates (via phosphoinositide 3 kinase [PI3K]

195 and protein kinase B [AKT]) into mTORC1 signaling to regulating proliferation (Figure 4A). To explore the antiviral efficiency of targeting proteins downstream of GFRs, we first tested the PI3K inhibitors pictilisib and omipalisib (Ippolito et al., 2016; Sarker et al., 2015; Schmid et al., 2016). Both compounds inhibited viral replication, based on their propensity to prevent cytopathogenic e $\square$ ect (CPE) and viral RNA production in cells (Figure 4B-D, S5, and S6). Our drug-target

200 analyses identified mitogen activated protein kinase kinase (MAP2K2, better known as MEK) and the RAF inhibitor sorafenib (Wilhelm et al., 2006) as promising targets inhibiting downstream signaling of GFRs (Figure 4A). Thus, we tested sorafenib and the dual RAF/MEK inhibitor RO5126766 in our viral replication assays. Both compounds inhibited cytopathic effects during infection and the viral replication (Figure 4B-D, S5, and S6). To validate our findings in another

205 cell line, we repeated the treatments in UKF-RC-2 cells infected with SARS-CoV-2. Quantifying the viral RNA copies in the supernatant, we observed that the compounds efficiently inhibited virus replication (Figure 4E and S7A) at non-toxic conditions (Figure S7B, except for sorafenib). Overall, five compounds, inhibiting downstream signaling of GFRs, prevented SARS-CoV-2 replication at clinically achievable concentrations (Figure 4B and 5) (Eskens et al., 2001; Fucile

210 et al., 2014; Martinez-Garcia et al., 2012; Munster et al., 2016; Sarker et al., 2015), emphasizing the importance of GFR signaling during SARS-CoV-2 infection and revealing clinically available treatment options as drug candidates for COVID-19.

\section{Discussion}

With the rapid spreading of the COVID-19 pandemic, investigating the molecular mechanisms

215 underlying SARS-CoV-2 infection are of high importance. In particular, the processes underlying infection and host-cell response remain unclear. These would offer potential avenues for pharmacological treatment of COVID-19. Here, we report global, differential phosphorylation analysis of host cells after infection with intact SARS-CoV-2 virus. We could identify phosphorylation sites on numerous viral proteins in cells, showing that they can undergo efficient

220 modification in infected cells. Until now, we can only speculate about the host kinases involved and the functions driven by PTMs, which will be an important topic for follow-up studies. For SARS-CoV-1, it was shown that modification of viral proteins can lead to regulation of RNA binding of the nucleoprotein (Wu et al., 2009) and is needed for viral replication. Although similar effects in SARS-CoV-2 are likely, this remains to be studied in this novel virus. A recent paper

225 analyzed the interaction profile of SARS-CoV-2 proteins expressed in HEK293T cells (Gordon et al., 2020). For the heavily phosphorylated nucleoprotein they could identify interactions the host casein kinases, which might indicate possible modification events by the latter. Also for the ORF9b/protein $9 \mathrm{~b}$ that we found modified in cells, interaction mapping identified MARK kinases as interaction partners.

230 By exploring the signaling changes inside the host cell, we could gain important insights into host cell signaling during infection. We found essential GFR signaling pathways activated such as EGFR or PDGFR, together with a plethora of RhoGTPase associated signaling molecules. We could furthermore show modulation of the splicing machinery, in line with previous results 
indicating dependency of viral in vitro pathology on the host spliceosome (Bojkova et al., 2020).

The same is true for metabolic reprogramming, for which we found differential post-translational modification of most members of the carbon metabolic pathways, namely glycolysis, pentose phosphate and TCA cycle. These metabolic pathways were significantly up-regulated on total protein levels in the presented dataset, consistent with our previous study (Bojkova et al., 2020), suggesting that these key pathways are regulated on multiple levels.

240 A number of drugs to treat COVID-19 have been suggested, largely based on bioinformatics analyses of genetics or cellular data (Gordon et al., 2020; Li et al., 2020; Wang, 2020). However, for many of these compounds, studies explaining their working mechanisms in the context of SARS-CoV-2 or viral assays to determine their efficacy of blocking viral replication in cell models of SARS-CoV-2 infection, are missing. While monitoring signaling changes in host cells, we

245 observed activation of GFR signaling cascades after infection, consistent with other viruses relying on the receptors themselves or elicited signal transduction (Eierhoff et al., 2010; Kung et al., 2011; Lupberger et al., 2011; Ueki et al., 2013; Wu et al., 2017; Zhu et al., 2009). From our data we could not clearly conclude which GFR might be activated and thus tested whether GFR downstream signaling inhibition can prevent SARS-CoV-2 replication, as reported for some other 250 viruses (Baturcam et al., 2019; Pleschka et al., 2001). Previously, temporal kinome analysis identified antiviral potential of RAS/RAF/MEK and PI3K/AKT/ for MERS-CoV (Kindrachuk et al., 2015). By targeting the RAS/RAF/MEK and PI3K/AKT/mTOR downstream axes of GFR signaling, we found efficient inhibition of viral replication in two different cell lines derived from different tissues (Figure 4). GFR signaling was shown to play a role in diverse virus infections as

255 well as in fibrosis induction by SARS-CoV-1 (Beerli et al., 2019; Kung et al., 2011; Lupberger et al., 2011; Pleschka et al., 2001; Ueki et al., 2013; Venkataraman et al., 2017). Thus, our results in cytopathic effects might indeed indicate cytoprotective roles for GFR signaling axes during SARS-CoV-2 infection and possible development of fibrosis (Luo et al., 2020). Notably, some inhibitors used in our study such as omipalisib were shown to suppress fibrosis progression in

260 patients with idiopathic pulmonary fibrosis, which may share deregulation of signaling pathways involved in lung fibrosis of coronavirus patients (Venkataraman et al., 2017). These findings suggest that inhibitors of GFR downstream signaling may bring benefit to COVID-19 patients independently of their antiviral activity.

Taken together, this study provides new insights into molecular mechanisms elicited by SARS-

265 CoV-2 infection. Proteomic analyses revealed several pathways that are rearranged during infection and showed that targeting of those pathways is a valid strategy to inhibit cytopathic effects triggered by infection.

\section{Limitations}

In this study, cancer cell lines were used to assess the effect of SARS-CoV-2 on host cells

270 during infections. We chose the experimental time-point, based on previous analysis of the infection course in these cells (Bojkova et al., 2020). Notably, the kinetics of infection are likely to be different in other cell lines or primary material, since we also observed a different $\mathrm{MOI}$ needed for UKF-RC-2 cells. In addition, we tested the efficiency of the presented drugs only in the context of in vitro cell line experiments. Thus, the results do not represent direct evidence for the

275 use of these therapeutics in patients, as the effects might differ in primary tissue. The results presented indicate potential anti-viral effects that have to be further validated in other models and clinical trials to assess their usefulness for the treatment of COVID-19.

\section{Acknowledgements}

We thank the Quantitative Proteomics Unit (IBC2, Goethe University Frankfurt) for support and

280 expertise on LC-MS instrumentation and data analysis and Christiane Pallas and Lena Stegmann for support of with experimental work. 


\section{Funding}

C.M. was supported by the European Research Council under the European Union's Seventh Framework Programme (ERC StG 803565), CRC1177 and the Emmy Noether Programme of the Deutsche Forschungsgemeinschaft (DFG, MU 4216/1-1), the Johanna Quandt Young Academy at Goethe, Aventis Foundation Bridge Award, and the Goethe Corona Fonds. J.C. was funded by the Hilfe für krebskranke Kinder Frankfurt e.V. and the Frankfurter Stiftung für krebskranke Kinder.

\section{Author contributions}

290 Conceptualization, K.K., D.B., C.M., and J.C.; Methodology, K.K. and D.B.; Software, K.K.; Formal Analysis, K.K. and G.T.; Investigation, K.K., D.B., and J.C.; Data Curation, K.K.; Writing Original Draft, K.K. and C.M.; Writing - Review and Editing, K.K., D.B., G.T., C.M., and J.C.; Visualization, K.K.; Supervision, C.M. and J.C.; Project Administration, C.M. and J.C.; Funding Acquisition, S.C., C.M., and J.C.

\section{Competing interest}

The authors filed a patent application on the use of GFR signaling inhibitors for the treatment of COVID-19. 


\section{Figure Legends}

Fig. 1. Phospho proteomic profiling of SARS-CoV-2 infected cells.

(A) Experimental scheme. Caco-2 cells were infected with SARS-CoV-2 for one hour (MOI: 1), washed and incubated for additional 24 hours. Proteins were extracted and prepared for bottomup proteomics. All ten conditions were multiplexed using TMT10 reagents. $250 \mu \mathrm{g}$ of pooled samples were used for whole cell proteomics (24 Fractions) and the remainder ( 1 mg) enriched

305 for phosphopeptides by Fe-NTA. Phosphopeptides were fractionated into 8 fractions and concatenated into 4 fractions. All samples were measured on an Orbitrap Fusion Lumos.

(B) Volcano plot showing fold changes of infected versus mock cells for all quantified phosphopeptides. $\mathrm{P}$ values were calculated using an unpaired, two-sided student's t-test with equal variance assumed and adjusted using the Benjamini Hochberg FDR method $(\mathrm{N}=5$

310 biological replicates). Orange or blue points indicate significantly increased or decreased phosphopeptides, respectively.

(C) Volcano plot showing differences between SARS-CoV-2 and mock infected cells in total protein levels for all quantified proteins. $P$ values were calculated using an unpaired, two-sided student's t-test with equal variance assumed and adjusted using the Benjamini Hochberg FDR

315 method $(\mathrm{N}=5)$. Orange or blue points indicate significantly increased or decreased phosphopeptides, respectively.

(D) Distribution of phosphorylation sites identified across modified amino acids. See also Figure S1 and Tables S1 and S2.

(E - K) Domain structures of SARS-CoV-2 proteins predicted by InterPro. Identified

320 phosphorylation sites are indicated. Protein 3a (E), Membrane Protein M (F), Non-structural protein $6(\mathbf{G})$, Protein 9b $(\mathbf{H})$, Replicase Polyprotein $1 \mathrm{~b}(\mathbf{I})$ and Nucleoprotein N (J). (K) X-ray structure of the RNA binding domain (PDB: 6vyo, residues 47-173) with identified phosphorylation sites marked in red.

See also Table S1, S2, S3 and Figure S1.

325 Fig. 2. Correlation of co-regulated proteins identifies cellular signaling pathways modulated upon infection.

(A) Correlation map of all detected phospho-proteins indicating Euclidean distance between proteins. To determine correlation, Z-scores of phospho-peptides and total protein levels were added and all peptide values for one protein collapsed into an average $Z$ score. Correlation

330 clustering was performed by Euclidean distance on combined $Z$ scores for all conditions. Red dashed line indicates main clusters found and identified.

(B) Reactome pathway enrichment of proteins found in Cluster I in (A). Shown are the number of proteins identified in the respective cluster versus statistical significance of enrichment. Circles are increasingly sized according to the number of proteins found in the pathway.

335 (C) Scatter plot showing fold changes of phospho-peptides compared to fold changes of total protein levels. The yellow oval indicates peptides for which phosphorylation is not driven by changes in protein abundance.

(D) Reactome pathways found enriched in Cluster II in (A). Analyses and presentation as in (B).

(E) Scatter plot showing correlation between fold changes of phosphopeptides compared to fold changes of total proteins levels. Two subsets of phosphopeptides were detected: one was 
mainly regulated by differential modification (indicated in yellow), the second by changes in protein abundance.

(F) STRING network analysis of proteins decreased in total protein levels (Figure 1C). Inserts indicate pathways found in the network.

Fig.3. Drug-target phosphoprotein network analysis identifies growth factor signaling as central hub for possible intervention by repurposed drugs.

(A) Proteins significantly increased in phosphorylation (FC $>1$, FDR $<0.05$ ) were subjected to ReactomeFI pathway analysis and overlaid with a network of FDA-approved drugs. The network

350 was filtered for drugs and drug targets only, to identify pathways that could be modulated by drug repurposing. Red lines indicate drug-target interactions, grey lines protein-protein interactions. Identified drugs are represented with yellow rectangles, while proteins are represented by blue circles.

(B) Search across all proteins with significant phosphorylation changes upon SARS-CoV-2 infection for proteins related to the EGFR pathway. STRING network highlighting all proteins annotated for EGFR signaling and their direct interaction neighbors. Red lines indicate direct EGFR interactions,black lines indicate interactions between pathway members. Grey lines represent filtered interactions to represent the whole network.

(C) Pathway representation of proteins identified in (B) to be direct functional interactors of

360 EGFR, according to the STRING interaction database (confidence cutoff 0.9). Phosphorylation changes of all significantly regulated sites are indicated by color-coded pie charts. Red indicates upregulation and blue indicates down-regulation.

See also Figure S4.

Fig. 4. Inhibition of growth factor receptor downstream signaling prevents SARS-CoV-2 replication.

(A) Schematic representation of growth factor signaling pathways activated upon SARS-CoV-2 infection. Inhibitors tested are indicated and their targets shown.

(B) Viral replication assay. Percentage inhibition of cytopathic effects (CPE) is plotted versus compound concentration ( $\mathrm{N}=3$ biological replicates for all compounds). Grey dots indicate

370 replicate measurements, red lines dose-response curve fits.

(C) Quantification of viral RNA in the supernatant of Caco-2 cells. Supernatant of control cells, infected cells (MOI 0.01) and infected cells treated either with Pictilisib, Omipalisib, Sorafenib, R05126766 or Lorafenib at indicated concentrations was analyzed by quantitative PCR for viral genome. $\mathrm{N}=3$, bar indicates mean of replicates, error bars indicate SD.

375 (D) Microscopy pictures showing staining for dsRNA to determine viral dsRNA production and CPE. Mock or SARS-CoV-2 infected cells are shown on the left. SARS-CoV-2 infected cells were treated with different concentrations of inhibitors (as indicated) and imaged after 24 hours. Pictilibsib: $0.625 \mu \mathrm{M}, 2.5 \mu \mathrm{M}, 10 \mu \mathrm{M}$; omipalisib: $0.01 \mu \mathrm{M}, 0.625 \mu \mathrm{M}, 2.5 \mu \mathrm{M}$; sorafenib: $2.5 \mu \mathrm{M}$, $5 \mu \mathrm{M}, 10 \mu \mathrm{M}$; RO5126766: $2.5 \mu \mathrm{M}, 5 \mu \mathrm{M}, 10 \mu \mathrm{M}$; lonafarnib: $0.6 \mu \mathrm{M}, 2.5 \mu \mathrm{M}, 10 \mu \mathrm{M}$. N = 3

380 technical replicates, one representative picture is shown, two more areas of the same well are shown in Figure S4. Scale bar represents $100 \mu \mathrm{m}$.

(E) Quantification of viral RNA in the supernatant of UKF-RC-2 cells. Supernatant of control cells, SARS-CoV-2 infected cells (MOI 0.1) untreated or treated with Pictilisib, Omipalisib, 
Sorafenib, RO5126766 or Lorafenib at indicated concentrations were analyzed by quantitative PCR for viral genome. $\mathrm{N}=3$ biological replicates, bar indicates mean of replicates, error bars indicate SD.

See also Figure S5.

Fig. 5. Effect of growth factor signaling on SARS-CoV-2 replication.

Upon infection growth factor signaling is activated and leads among others to the induction of 390 Phosphoinositol 3 Kinase (PI3K) and Mitogen Activated Protein Kinase (MAPK) signaling events. Inhibition of either axis of the two (by Sorafenib, RP5126766, Lonafarnib, Pictilisib or Omapalisib) leads to decreased replication of SARS-CoV-2 inside the host cell. 


\section{Resource Availability}

\section{Lead contact}

Further information and requests for resources and reagents should be directed to and will be fulfilled by the lead contact:

Christian Münch: ch.muench@em.uni-frankfurt.de (C.M.)

\section{Materials Availability}

UKF-RC-2 human kidney cells derived from renal carcinoma are available upon request.

\section{Data and Code Availability}

The mass spectrometry proteomics data have been deposited to the ProteomeXchange

405 Consortium via the PRIDE (Perez-Riverol et al., 2019) partner repository with the dataset identifiers PXD018357. Annotated spectra of new SARS-CoV-2 phospho-peptides were deposited at Mendeley Data under https://doi.org/10.17632/4g2zzdfd47.1 . Additional supplementary items are available under https://doi.org/10.17632/kpnzwh6v6j.1 .

\section{Experimental model and subject details}

\section{Cell culture}

Human Caco-2 cells, derived from colon carcinoma, was obtained from the Deutsche Sammlung von Mikroorganismen und Zellkulturen (DSMZ; Braunschweig, Germany). Cells were grown at $37^{\circ} \mathrm{C}$ in Minimal Essential Medium (MEM) supplemented with $10 \%$ fetal bovine serum (FBS) and containing $100 \mathrm{IU} / \mathrm{ml}$ penicillin and $100 \mu \mathrm{g} / \mathrm{ml}$ streptomycin. All culture reagents were purchased

415 from Sigma.

Cell line designated UKF-RC-2 was established from a tumor sample of a patient with a diagnosis of renal carcinoma hospitalized at Department of Urology, University Hospital Frankfurt. Tumor tissue was cut in pieces and dissociated using $0.2 \%$ trypsin solution. Primary tumor cells and passaging of cell line was performed using IMDM medium supplemented with

$42010 \%$ FCS and antibiotics. UKF-RC-2 cells between passages 15 and 20 were used for antiviral experiments. All culture reagents were purchased from Sigma.

\section{Virus preparation}

SARS-CoV-2 was isolated from samples of travelers returning from Wuhan (China) to Frankfurt (Germany) using human colon carcinoma cell line $\mathrm{CaCo}-2$ as described previously ${ }^{12}$. SARS-

425 CoV-2 stocks used in the experiments had undergone one passage on CaCo-2 cells and were stored at $-80^{\circ} \mathrm{C}$. Virus titers were determined as TCID50/ml in confluent cells in 96-well microtiter plates.

\section{Method details}

\section{Antiviral and cell viability assays}

430 Confluent layers of CaCo-2 cells in 96-well plates were infected with SARS-CoV-2 at MOI 0.01. Virus was added together with drugs and incubated in MEM supplemented with $2 \%$ FBS with different drug dilutions. Cytopathogenic e $\square$ ect (CPE) was assessed visually $48 \mathrm{~h}$ after infection. To assess effects of drugs on Caco-2 cell viability, confluent cell layers were treated with different drug concentration in 96-well plates. The viability was measured using the Rotitest 
Vital (Roth) according to manufacturer's instructions. Data for each condition was collected for at least three biological replicates. For dose response curves, data was fitted with all replicates using OriginPro 2020 with the following equation:

$y=A 1+\frac{A 2-A 1}{1+10^{(L O G x 0-x) p}}$

IC50 values were generated by OriginPro 2020 together with metrics for curve fits.

440 For UKF-RC-2 cells, the assay was performed as described above, except for usage of a MOI of 0.1 as staining experiments for SARS-CoV-2 infection in UKF-RC-2 cells revealed the need of a higher $\mathrm{MOI}$ to achieve comparable effects to $\mathrm{CaCo}-2$ cells.

\section{Quantification of viral RNA}

SARS-CoV-2 RNA from cell culture supernatant samples was isolated using AVL buffer and the

445 QIAamp Viral RNA Kit (Qiagen) according to the manufacturer's instructions. Absorbance-based quantification of the RNA yield was performed using the Genesys 10S UV-Vis

Spectrophotometer (Thermo Scientific). RNA was subjected to OneStep qRT-PCR analysis using the Luna Universal One-Step RT-qPCR Kit (New England Biolabs) and a CFX96 RealTime System, C1000 Touch Thermal Cycler. Primers were adapted from the WHO protocol

450 (Corman et al., 2020) targeting the open reading frame for RNA-dependent RNA polymerase (RdRp): RdRP_SARSr-F2 (GTG ARA TGG TCA TGT GTG GCG G) and RdRP_SARSr-R1 (CAR ATG TTA AAS ACA CTA TTA GCA TA) using $0.4 \mu \mathrm{M}$ per reaction. Standard curves were created using plasmid DNA ( $\mathrm{pEX}-\mathrm{A} 128-\mathrm{RdRP}$ ) harboring the corresponding amplicon regions for RdRP target sequence according to GenBank Accession number NC_045512. All quantification experiments have been carried out with biological replicates.

\section{Detection of viral load by microscopy}

Effect of selected compounds on viral replication was assessed by staining of double-stranded RNA, which has been shown to be sufficient for measurement of SARS-CoV-1 replication(Weber et al., 2006). Briefly, cells were fixed with acetone/methanol (40:60) solution $48 \mathrm{~h}$ post infection. Immunostaining was performed using a monoclonal antibody directed against dsRNA (1:150 dilution, SCICONS J2, mouse, IgG2a, kappa chain, English \& Scientific Consulting Kft., Szirák, Hungary), which was detected with biotin-conjugated secondary antibody (1:1000 dilution, Jackson ImmunoResearch) followed by application streptavidin, peroxidase conjugate (1:3000 dilution, Sigma Aldrich). Lastly, the dsRNA positive cells were visualized by addition of AEC

465 substrate. Wells were imaged at different areas to visualize a larger area (presented in Supplementary figures).

\section{Sample preparation for mass spectrometry}

For all proteomics analysis, Caco- 2 cells were infected at an $\mathrm{MOI}$ of 1 and the sample preparation was performed as described previously (Klann et al., 2020). Briefly, lysates were 470 precipitated by methanol/chloroform and proteins resuspended in $8 \mathrm{M}$ Urea/10 mM EPPS pH 8.2. Concentration of proteins was determined by Bradford assay and $300 \mu \mathrm{g}$ of protein per samples was used for digestion. For digestion, the samples were diluted to $1 \mathrm{M}$ Urea with $10 \mathrm{mM}$ EPPS pH 8.2 and incubated overnight with 1:50 LysC (Wako Chemicals) and 1:100 Sequencing grade trypsin (Promega). Digests were acidified using TFA and tryptic peptideswere purified by

475 tC18 SepPak (50 mg, Waters). $125 \mu \mathrm{g}$ peptides per sample were TMT labelled and the mixing was normalized after a single injection measurement by LC-MS/MS to equimolar ratios for each channel. $250 \mu \mathrm{g}$ of pooled peptides were dried for offline High pH Reverse phase fractionation by HPLC (whole cell proteome) and remaining $1 \mathrm{mg}$ of multiplexed peptides were used for phospho-peptide enrichment by High-Select Fe-NTA Phosphopeptide enrichment kit (Thermo 
480 Fisher) after manufacturer`s instructions. After enrichment, peptides were dried and resuspended in $70 \%$ acetonitrile/0.1\% TFA and filtered through a C 8 stage tip to remove contaminating Fe-NTA particles. Dried phospho-peptides then were fractionated on C18 (Empore) stage-tip. For fractionation C18 stagetips were washed with $100 \%$ acetonitrile twice, followed by equilibration with $0.1 \%$ TFA solution. Peptides were loaded in $0.1 \%$ TFA solution and

485 washed with water. Elution was performed stepwise with different acetonitrile concentrations in $0.1 \%$ Triethylamine solution $(5 \%, 7.5 \%, 10 \%, 12.5 \%, 15 \%, 17.5 \%, 20 \%, 50 \%)$. The eight fractions were concatenated into four fractions and dried for LC-MS.

\section{Offline high $\mathrm{pH}$ reverse phase fractionation}

Peptides were fractionated using a Dionex Ultimate 3000 analytical HPLC. $250 \mu \mathrm{g}$ of pooled and 490 purified TMT-labeled samples were resuspended in $10 \mathrm{mM}$ ammonium-bicarbonate (ABC), $5 \%$ ACN, and separated on a $250 \mathrm{~mm}$ long $\mathrm{C} 18$ column (X-Bridge, $4.6 \mathrm{~mm}$ ID, $3.5 \mu \mathrm{m}$ particle size; Waters) using a multistep gradient from $100 \%$ Solvent A ( $5 \%$ ACN, $10 \mathrm{mM} \mathrm{ABC}$ in water) to $60 \%$ Solvent B $(90 \%$ ACN, $10 \mathrm{mM} \mathrm{ABC}$ in water) over $70 \mathrm{~min}$. Eluting peptides were collected every $45 \mathrm{~s}$ into a total of 96 fractions, which were cross-concatenated into 24 fractions and dried for

495 further processing.

\section{Liquid chromatography mass spectrometry}

All mass spectrometry data was acquired in centroid mode on an Orbitrap Fusion Lumos mass spectrometer hyphenated to an easy-nLC 1200 nano HPLC system using a nanoFlex ion source (ThermoFisher Scientific) applying a spray voltage of $2.6 \mathrm{kV}$ with the transfer tube heated to

$500300^{\circ} \mathrm{C}$ and a funnel RF of $30 \%$. Internal mass calibration was enabled (lock mass 445.12003 $\mathrm{m} / \mathrm{z}$ ). Peptides were separated on a self-made, $32 \mathrm{~cm}$ long, $75 \mu \mathrm{m}$ ID fused-silica column, packed in house with $1.9 \mu \mathrm{m} \mathrm{C18}$ particles (ReproSil-Pur, Dr. Maisch) and heated to $50^{\circ} \mathrm{C}$ using an integrated column oven (Sonation). HPLC solvents consisted of $0.1 \%$ Formic acid in water (Buffer A) and $0.1 \%$ Formic acid, $80 \%$ acetonitrile in water (Buffer B).

505 For total proteome analysis, a synchronous precursor selection (SPS) multi-notch MS3 method was used in order to minimize ratio compression as previously described (McAlister et al., 2014). Individual peptide fractions were eluted by a non-linear gradient from 7 to $40 \%$ B over 90 minutes followed by a step-wise increase to $95 \%$ B in 6 minutes which was held for another 9 minutes. Full scan MS spectra (350-1400 m/z) were acquired with a resolution of $120,000 \mathrm{at} \mathrm{m} / \mathrm{z}$

510200 , maximum injection time of $100 \mathrm{~ms}$ and AGC target value of $4 \times 10^{5}$. The 20 most intense precursors with a charge state between 2 and 6 per full scan were selected for fragmentation ("Top 20") and isolated with a quadrupole isolation window of 0.7 Th. MS2 scans were performed in the lon trap (Turbo) using a maximum injection time of $50 \mathrm{~ms}$, AGC target value of $1.5 \times 10^{4}$ and fragmented using CID with a normalized collision energy (NCE) of $35 \%$. SPS-MS3

515 scans for quantification were performed on the 10 most intense MS2 fragment ions with an isolation window of $0.7 \mathrm{Th}$ (MS) and $2 \mathrm{~m} / \mathrm{z}$ (MS2). lons were fragmented using HCD with an NCE of $65 \%$ and analyzed in the Orbitrap with a resolution of 50,000 at $\mathrm{m} / \mathrm{z} 200$, scan range of 110 $500 \mathrm{~m} / \mathrm{z}$, AGC target value of $1.5 \times 10^{5}$ and a maximum injection time of $120 \mathrm{~ms}$. Repeated sequencing of already acquired precursors was limited by setting a dynamic exclusion of 45

520 seconds and 7 ppm and advanced peak determination was deactivated.

For phosphopeptide analysis, each peptide fraction was eluted by a linear gradient from 5 to $32 \%$ B over 120 minutes followed by a step-wise increase to $95 \%$ B in 8 minutes which was held for another 7 minutes. Full scan MS spectra (350-1400 m/z) were acquired with a resolution of 
120,000 at $\mathrm{m} / \mathrm{z} 200$, maximum injection time of $100 \mathrm{~ms}$ and $\mathrm{AGC}$ target value of $4 \times 10^{5}$. The 20 most intense precursors per full scan with a charge state between 2 and 5 were selected for fragmentation ("Top 20"), isolated with a quadrupole isolation window of 0.7 Th and fragmented via HCD applying an NCE of $38 \%$. MS2 scans were performed in the Orbitrap using a resolution of 50,000 at $\mathrm{m} / \mathrm{z} 200$, maximum injection time of $86 \mathrm{~ms}$ and $A G C$ target value of $1 \times 10^{5}$. Repeated sequencing of already acquired precursors was limited by setting a dynamic exclusion

530 of 60 seconds and 7 ppm and advanced peak determination was deactivated. $\mathrm{An} \mathrm{MS}^{2}$ based method was chosen, because of higher precision and identification rates (Hogrebe et al., 2018). Phospho-peptide fractions intrinsically exhibit lower complexity, rendering them less prone to ratio compression by isolation interference.

\section{Mass spectrometry data analysis}

535 Raw files were analyzed using Proteome Discoverer (PD) 2.4 software (ThermoFisher Scientific). Spectra were selected using default settings and database searches performed using SequestHT node in PD. Database searches were performed against trypsin digested Homo Sapiens SwissProt database, SARS-CoV-2 database (Uniprot pre-release). Static modifications were set as TMT6 at the N-terminus and lysines and carbamidomethyl at cysteine residues.

540 Search was performed using Sequest HT taking the following dynamic modifications into account: Oxidation (M), Phospho (S, T, Y), Met-loss (Protein N-terminus), Acetyl (Protein Nterminus) and Met-loss acetyl (Protein $\mathrm{N}$-terminus). For whole cell proteomics, the same settings were used except phosphorylation was not allowed as dynamic modification. For phosphoproteomics all peptide groups were normalized by summed intensity normalization and then

545 analyzed on peptide level. For whole cell proteomics normalized PSMs were summed for each accession and data exported for further use. Peptide and protein identifications were validated using a concatenated target-decoy strategy and FDR was estimated using q-values calculated by Percolator applying $1 \%$ and $5 \%$ cut-offs for high and medium confidence hits, while only high confident proteins are reported. Phosphosite localization probabilities were calculated using the ptmRS-node working in "PhosphoRS mode" and using default settings.

\section{Quantification and statistical analysis}

\section{Significance testing}

Unless otherwise stated significance was tested by unpaired, two-sided students t-tests with

555 equal variance assumed. Resulting $P$ values were corrected using the Benjamini-Hochberg FDR procedure. Adjusted $\mathrm{P}$ values smaller/equal 0.05 were considered significant. For phosphoproteomics an additional fold change cutoff was applied (log2 $>|1|)$, while for total protein levels, due to different dynamic range, a fold change cutoff of $\log 2>|0.5|$ was applied.

\section{Prediction of kinase motifs}

560 Kinase motifs of phosphopeptides from SARS-CoV-2 proteins were predicted using NetPhos 3.1 (Blom et al., 1999) and GPS 5.0 (stand-alone version) using the fasta-file of the Uniprot prerelease which was also used for the proteomics data analysis.(Blom et al., 2004; Wang et al., 2020). For NetPhos, only Kinases with a score above 0.5 were considered as positive hits. For GPS 5.0, sequences were submitted separately for S/T- and Y-kinases and the score threshold 565 was set to "high". For the final list in Supplementary Table 3, only the top hits with the highest scores were considered. 


\section{Journal Pre-proof}

\section{Protein co-regulation analysis}

Z-scores were calculated for each phospho-site and the total protein levels individually.

Phosphosites were collapsed by average. For merging phosphorylation and total protein levels

570 Z-scores for collapsed phosphorylation and protein level were added for each condition and replicate. Thus, both negative Z-scores (downregulation) will produce a lower combined Z-score and vice versa two positive Z-scores will produce a larger combined Z-score. Next, Euclidean distance correlation for all possible protein-protein pairs were calculated, taking all conditions and replicates individually into account. A heatmap was then build by Euclidean distance

575 hierarchical clustering of the correlation matrix.

\section{Pathway enrichment analysis}

Pathway enrichment analysis was performed by ReactomeFI cytoscape plugin or by STRING functional enrichment analysis. Both analysis used Reactome database for pathway annotations.

\section{Drug-target network analysis}

580 All proteins were loaded into ReactomeFI cytoscape plugin to visualize protein-protein functional interaction network. Next, drugs were overlaid by ReactomeFI and network was filtered for the drugs and the first interacting partners. Layout was calculated by yFilesLayout algorithm.

\section{Interaction network analysis}

All proteins showing significant regulation were loaded by OmicsVisualizer cytoscape plugin and

585 STRING interaction network was retrieved with a confidence cutoff of 0.9. For EGFR subnetwork, EGFR was selected with first interacting neighbors. 


\section{Supplementary Table Legends}

Supplementary Table 1: Quantification of phospho-peptide data, Related to Figure 1, 2 and 3.

Shown are Protein UniProt accessions together with gene names and modification sites (numbers in brackets indicate localization confidence). Log2 ratios between mock and SARSCoV-2 infected cells were calculated together with $P$ values. $P$ values were adjusted using Benjamini Hochberg FDR adjustment. Additionally, replicate quantification values are given.

Supplementary Table 2: Quantifications of total protein levels of SARS-CoV-2 infected cells and control cells, Related to Figure 1, 2 and 3.

Uniprot Accessions, Protein description, number of PSMs used for quantification, gene symbol, replicate quantifications, log2 ratio and adjusted $P$ values are shown. $P$ values were adjusted using Benjamini Hochberg FDR adjustment.

\section{Supplementary Table 3: Viral modification sites, Related to Figure 1.}

Modified amino acid, position in peptide, site probability, peptide sequence, number of modified PSMs, unmodified PSMs, protein accession, protein description, position in protein and modification motifs are given for all identified viral modification sites. Additionally, results of kinase predictions by NetPhos 3.1 and GPS5 are added.

605 Supplementary Table 4: Reactome Pathway enrichment analysis for proteins found belonging to clusters identified in correlation analysis, Related to Figure 2.

Reactome pathway, number of genes found in pathway, enrichment FDR and individual genes in pathway are given for all identified clusters.

Supplementary Table 5: Reactome Pathway enrichment analysis for proteins found significantly decreased in total protein levels, Related to Figure 1 and 2.

Reactome pathway, number of genes found in pathway, enrichment FDR and individual genes in pathway are given.

\section{References}

615 Baturcam, E., Vollmer, S., Schlüter, H., Maciewicz, R.A., Kurian, N., Vaarala, O., Ludwig, S., and Cunoosamy, D.M. (2019). MEK inhibition drives anti-viral defence in RV but not RSV challenged human airway epithelial cells through AKT/p70S6K/4E-BP1 signalling. Cell Commun. Signal. 17, 78.

Beerli, C., Yakimovich, A., Kilcher, S., Reynoso, G. V, Fläschner, G., Müller, D.J., Hickman,

620 H.D., and Mercer, J. (2019). Vaccinia virus hijacks EGFR signalling to enhance virus spread through rapid and directed infected cell motility. Nat. Microbiol. 4, 216-225.

Blom, N., Gammeltoft, S., and Brunak, S. (1999). Sequence and structure-based prediction of eukaryotic protein phosphorylation sites1 1Edited by F. E. Cohen. J. Mol. Biol. 294, 1351-1362.

Blom, N., Sicheritz-Pontén, T., Gupta, R., Gammeltoft, S., and Brunak, S. (2004). Prediction of 625 post-translational glycosylation and phosphorylation of proteins from the amino acid sequence. Proteomics 4, 1633-1649.

Bojkova, D., Klann, K., Koch, B., Widera, M., Krause, D., Ciesek, S., Cinatl, J., and Münch, C. (2020). Proteomics of SARS-CoV-2-infected host cells reveals therapy targets. Nature. 
Chen, N., Zhou, M., Dong, X., Qu, J., Gong, F., Han, Y., Qiu, Y., Wang, J., Liu, Y., and Wei, Y. pneumonia in Wuhan, China: a descriptive study. Lancet.

Corman, V.M., Landt, O., Kaiser, M., Molenkamp, R., Meijer, A., Chu, D.K., Bleicker, T., Brünink, S., Schneider, J., Schmidt, M.L., et al. (2020). Detection of 2019 novel coronavirus (2019-nCoV) by real-time RT-PCR. Euro Surveill. 25, 2000045.

635 Eierhoff, T., Hrincius, E.R., Rescher, U., Ludwig, S., and Ehrhardt, C. (2010). The epidermal growth factor receptor (EGFR) promotes uptake of influenza A viruses (IAV) into host cells. PLoS Pathog. 6, e1001099-e1001099.

Eskens, F.A.L.M., Awada, A., Cutler, D.L., de Jonge, M.J.A., Luyten, G.P.M., Faber, M.N., Statkevich, P., Sparreboom, A., Verweij, J., Hanauske, A.-R., et al. (2001). Phase I and

640 Pharmacokinetic Study of the Oral Farnesyl Transferase Inhibitor SCH 66336 Given Twice Daily to Patients With Advanced Solid Tumors. J. Clin. Oncol. 19, 1167-1175.

Fucile, C., Marenco, S., Bazzica, M., Zuccoli, M.L., Lantieri, F., Robbiano, L., Marini, V., Di Gion, P., Pieri, G., Stura, P., et al. (2014). Measurement of sorafenib plasma concentration by highperformance liquid chromatography in patients with advanced hepatocellular carcinoma: is it

645 useful the application in clinical practice? A pilot study. Med. Oncol. 32, 335.

Gordon, D.E., Jang, G.M., Bouhaddou, M., Xu, J., Obernier, K., O\&\#039;Meara, M.J., Guo, J.Z., Swaney, D.L., Tummino, T.A., Huttenhain, R., et al. (2020). A SARS-CoV-2-Human ProteinProtein Interaction Map Reveals Drug Targets and Potential Drug-Repurposing. BioRxiv 2020.03.22.002386.

650 Grimmler, M., Bauer, L., Nousiainen, M., Körner, R., Meister, G., and Fischer, U. (2005). Phosphorylation regulates the activity of the SMN complex during assembly of spliceosomal $U$ snRNPs. EMBO Rep. 6, 70-76.

Herzog, P., Drosten, C., and Müller, M.A. (2008). Plaque assay for human coronavirus NL63 using human colon carcinoma cells. Virol. J. 5, 138.

655 Hogrebe, A., von Stechow, L., Bekker-Jensen, D.B., Weinert, B.T., Kelstrup, C.D., and Olsen, J. $\mathrm{V}$ (2018). Benchmarking common quantification strategies for large-scale phosphoproteomics. Nat. Commun. 9, 1045.

Ilan, L., Osman, F., Namer, L.S., Eliahu, E., Cohen-Chalamish, S., Ben-Asouli, Y., Banai, Y., and Kaempfer, R. (2017). PKR activation and elF2 $\alpha$ phosphorylation mediate human globin mRNA 660 splicing at spliceosome assembly. Cell Res. 27, 688-704.

Ippolito, T., Tang, G., Mavis, C., Gu, J.J., Hernandez-Ilizaliturri, F.J., and Barth, M.J. (2016). Omipalisib (GSK458), a Novel Pan-PI3K/mTOR Inhibitor, Exhibits In Vitro Anti-Lymphoma Activity in Chemotherapy-Sensitive and -Resistant Models of Burkitt Lymphoma. Blood 128, 5376.

665 Kindrachuk, J., Ork, B., Hart, B.J., Mazur, S., Holbrook, M.R., Frieman, M.B., Traynor, D., Johnson, R.F., Dyall, J., Kuhn, J.H., et al. (2015). Antiviral Potential of ERK/MAPK and $\mathrm{PI}$ KK/AKT/mTOR Signaling Modulation for Middle East Respiratory Syndrome Coronavirus Infection as Identified by Temporal Kinome Analysis. Antimicrob. Agents Chemother. 59, 10881099.

670 Klann, K., Tascher, G., and Münch, C. (2020). Functional Translatome Proteomics Reveal Converging and Dose-Dependent Regulation by mTORC1 and elF2a. Mol. Cell 77, 913-925.e4. 
Kung, C.-P., Meckes, D.G., and Raab-Traub, N. (2011). Epstein-Barr Virus LMP1 Activates EGFR, STAT3, and ERK through Effects on PKCס. J. Virol. 85, 4399 LP - 4408.

X., Yu, J., Zhang, Z., Ren, J., Peluffo, A.E., Zhang, W , Zhao, Y., Yan, K., Cohen, D., and Wang, W. (2020). Network Bioinformatics Analysis Provides Insight into Drug Repurposing for COVID-2019.

Luo, W., Yu, H., Gou, J., Li, X., Sun, Y., Li, J., and Liu, L. (2020). Clinical Pathology of Critical Patient with Novel Coronavirus Pneumonia (COVID-19).

Lupberger, J., Zeisel, M.B., Xiao, F., Thumann, C., Fofana, I., Zona, L., Davis, C., Mee, C.J., 680 Turek, M., Gorke, S., et al. (2011). EGFR and EphA2 are host factors for hepatitis $C$ virus entry and possible targets for antiviral therapy. Nat. Med. 17, 589-595.

Martinez-Garcia, M., Banerji, U., Albanell, J., Bahleda, R., Dolly, S., Kraeber-Bodéré, F., Rojo, F., Routier, E., Guarin, E., Xu, Z.-X., et al. (2012). First-in-Human, Phase I Dose-Escalation Study of the Safety, Pharmacokinetics, and Pharmacodynamics of RO5126766, a First-in-Class Dual MEK/RAF Inhibitor in Patients with Solid Tumors. Clin. Cancer Res. 18, 4806 LP - 4819.

Mathew, R., Hartmuth, K., Möhlmann, S., Urlaub, H., Ficner, R., and Lührmann, R. (2008). Phosphorylation of human PRP28 by SRPK2 is required for integration of the U4/U6-U5 trisnRNP into the spliceosome. Nat. Struct. Mol. Biol. 15, 435-443.

McAlister, G.C., Nusinow, D.P., Jedrychowski, M.P., Wühr, M., Huttlin, E.L., Erickson, B.K., Rad, 690 R., Haas, W., and Gygi, S.P. (2014). MultiNotch MS3 enables accurate, sensitive, and multiplexed detection of differential expression across cancer cell line proteomes. Anal. Chem.

Mermoud, J.E., Cohen, P.T., and Lamond, A.I. (1994). Regulation of mammalian spliceosome assembly by a protein phosphorylation mechanism. EMBO J. 13, 5679-5688.

Munster, P., Aggarwal, R., Hong, D., Schellens, J.H.M., van der Noll, R., Specht, J., Witteveen, 695 P.O., Werner, T.L., Dees, E.C., Bergsland, E., et al. (2016). First-in-Human Phase I Study of GSK2126458, an Oral Pan-Class I Phosphatidylinositol-3-Kinase Inhibitor, in Patients with Advanced Solid Tumor Malignancies. Clin. Cancer Res. 22, 1932 LP - 1939.

Perez-Riverol, Y., Csordas, A., Bai, J., Bernal-Llinares, M., Hewapathirana, S., Kundu, D.J., Inuganti, A., Griss, J., Mayer, G., Eisenacher, M., et al. (2019). The PRIDE database and related

700 tools and resources in 2019: improving support for quantification data. Nucleic Acids Res. 47, D442-D450.

Pleschka, S., Wolff, T., Ehrhardt, C., Hobom, G., Planz, O., Rapp, U.R., and Ludwig, S. (2001). Influenza virus propagation is impaired by inhibition of the Raf/MEK/ERK signalling cascade. Nat. Cell Biol. 3, 301-305.

705 Ren, X., Glende, J., Al-Falah, M., Vries, V. de, Schwegmann-Wessels, C., Qu, X., Tan, L., Tschernig, T., Deng, H., Naim, H.Y., et al. (2006). Analysis of ACE2 in polarized epithelial cells: surface expression and function as receptor for severe acute respiratory syndrome-associated coronavirus. J. Gen. Virol. 87, 1691-1695.

Sarker, D., Ang, J.E., Baird, R., Kristeleit, R., Shah, K., Moreno, V., Clarke, P.A., Raynaud, F.I.,

710 Levy, G., Ware, J.A., et al. (2015). First-in-Human Phase I Study of Pictilisib (GDC-0941), a Potent Pan-Class I Phosphatidylinositol-3-Kinase (PI3K) Inhibitor, in Patients with Advanced Solid Tumors. Clin. Cancer Res. 21, 77 LP - 86.

Schmid, P., Pinder, S.E., Wheatley, D., Macaskill, J., Zammit, C., Hu, J., Price, R., Bundred, N., Hadad, S., Shia, A., et al. (2016). Phase II Randomized Preoperative Window-of-Opportunity 
715 Study of the PI3K Inhibitor Pictilisib Plus Anastrozole Compared With Anastrozole Alone in Patients With Estrogen Receptor-Positive Breast Cancer. J. Clin. Oncol. 34, 1987-1994.

Smith, M., and Smith, J.C. (2020). Repurposing Therapeutics for COVID-19: SupercomputerBased Docking to the SARS-CoV-2 Viral Spike Protein and Viral Spike Protein-Human ACE2 Interface.

720 Tangudu, C., Olivares, H., Netland, J., Perlman, S., and Gallagher, T. (2007). Severe acute respiratory syndrome coronavirus protein 6 accelerates murine coronavirus infections. J. Virol. $81,1220-1229$.

Ueki, I.F., Min-Oo, G., Kalinowski, A., Ballon-Landa, E., Lanier, L.L., Nadel, J.A., and Koff, J.L. (2013). Respiratory virus-induced EGFR activation suppresses IRF1-dependent interferon $\lambda$ and antiviral defense in airway epithelium. J. Exp. Med. 210, 1929-1936.

Venkataraman, T., and Frieman, M.B. (2017). The role of epidermal growth factor receptor (EGFR) signaling in SARS coronavirus-induced pulmonary fibrosis. Antiviral Res. 143, 142-150.

Venkataraman, T., Coleman, C., and Frieman, M. (2017). Overactive EGFR Signaling Leads to Increased Fibrosis After SARS-CoV Infection. J. Virol. JVI.00182-17.

730 Wang, J. (2020). Fast Identification of Possible Drug Treatment of Coronavirus Disease -19 (COVID-19) Through Computational Drug Repurposing Study.

Wang, C., Xu, H., Lin, S., Deng, W., Zhou, J., Zhang, Y., Shi, Y., Peng, D., and Xue, Y. (2020). GPS 5.0: An Update on the Prediction of Kinase-specific Phosphorylation Sites in Proteins. Genomics. Proteomics Bioinformatics.

735 Weber, F., Wagner, V., Rasmussen, S.B., Hartmann, R., and Paludan, S.R. (2006). Doublestranded RNA is produced by positive-strand RNA viruses and DNA viruses but not in detectable amounts by negative-strand RNA viruses. J. Virol. 80, 5059-5064.

Wilhelm, S., Carter, C., Lynch, M., Lowinger, T., Dumas, J., Smith, R.A., Schwartz, B., Simantov, R., and Kelley, S. (2006). Discovery and development of sorafenib: a multikinase inhibitor for 740 treating cancer. Nat. Rev. Drug Discov. 5, 835-844.

Wu, C.-H., Yeh, S.-H., Tsay, Y.-G., Shieh, Y.-H., Kao, C.-L., Chen, Y.-S., Wang, S.-H., Kuo, T.J., Chen, D.-S., and Chen, P.-J. (2009). Glycogen synthase kinase-3 regulates the phosphorylation of severe acute respiratory syndrome coronavirus nucleocapsid protein and viral replication. J. Biol. Chem. 284, 5229-5239.

745 Wu, Y., Prager, A., Boos, S., Resch, M., Brizic, I., Mach, M., Wildner, S., Scrivano, L., and Adler, B. (2017). Human cytomegalovirus glycoprotein complex $\mathrm{gH} / \mathrm{gL} / \mathrm{gO}$ uses PDGFR- $\alpha$ as a key for entry. PLoS Pathog. 13.

Yarden, Y. (2001). The EGFR family and its ligands in human cancer: signalling mechanisms and therapeutic opportunities. Eur. J. Cancer 37, 3-8.

750 Zhu, L., Lee, P., Lee, W., Zhao, Y., Yu, D., and Chen, Y. (2009). Rhinovirus-Induced Major Airway Mucin Production Involves a Novel TLR3-EGFR-Dependent Pathway. Am. J. Respir. Cell Mol. Biol. 40, 610-619. 
Highlights:

- Phosphoproteomics of SARS-CoV-2 infected cells reveal the signaling landscape

- SARS-CoV-2 proteins are extensively phosphorylated in host cells

- Infection leads to activation of growth factor receptor signaling

- Drugs inhibiting growth factor receptor signaling prevent viral replication

In this study, Klann et al. dissected the host cell signaling landscape upon infection with SARS-CoV-2. Mapping differential signaling networks identified a number of pathways activated during infection. Drug-target network analysis revealed potential therapeutic targets. Growth factor receptor signaling was highly activated upon infection and its inhibition prevented SARS-CoV-2 replication in cells. 
Figure 1

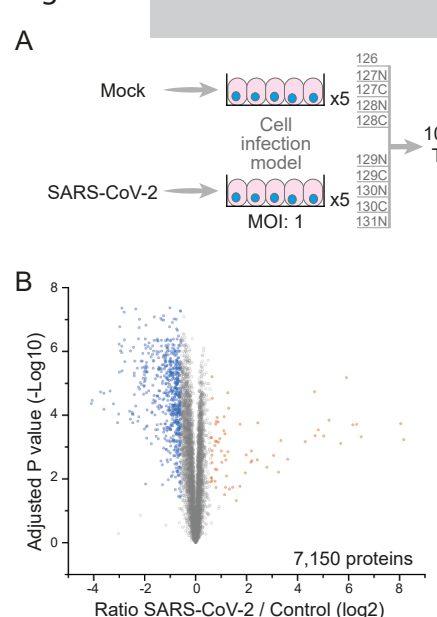

E

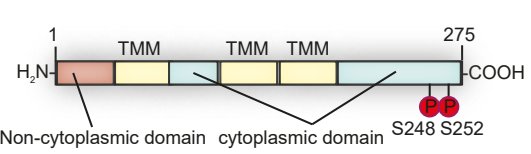

$\mathrm{H}$
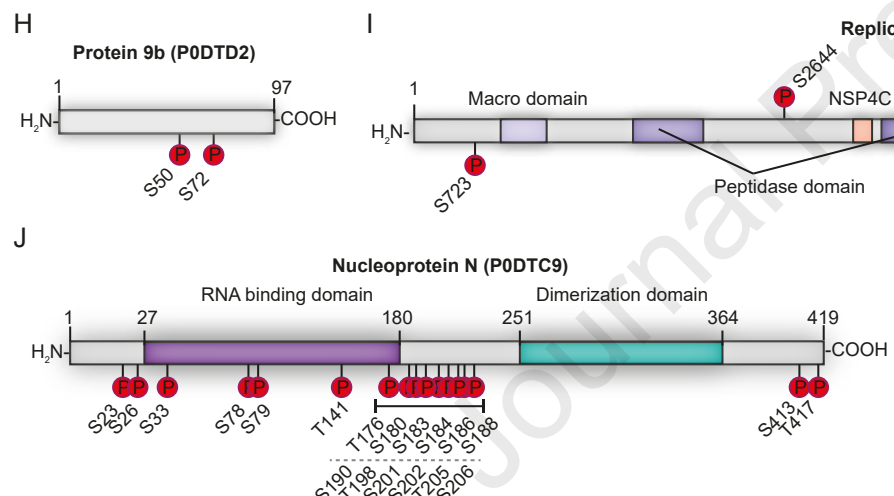

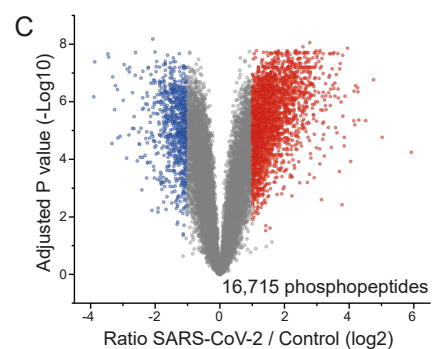

$\mathrm{F}$
D
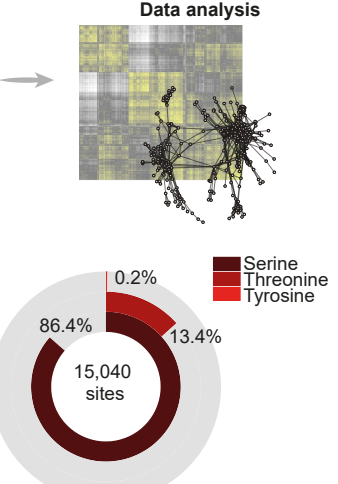

G Non-structural protein 6

Membrane Protein (PODTC5)
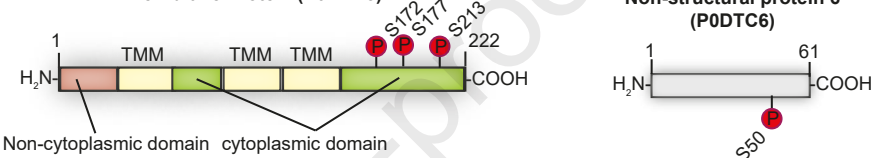

Replicase polyprotein 1b (PODTD1) 
Figure 2

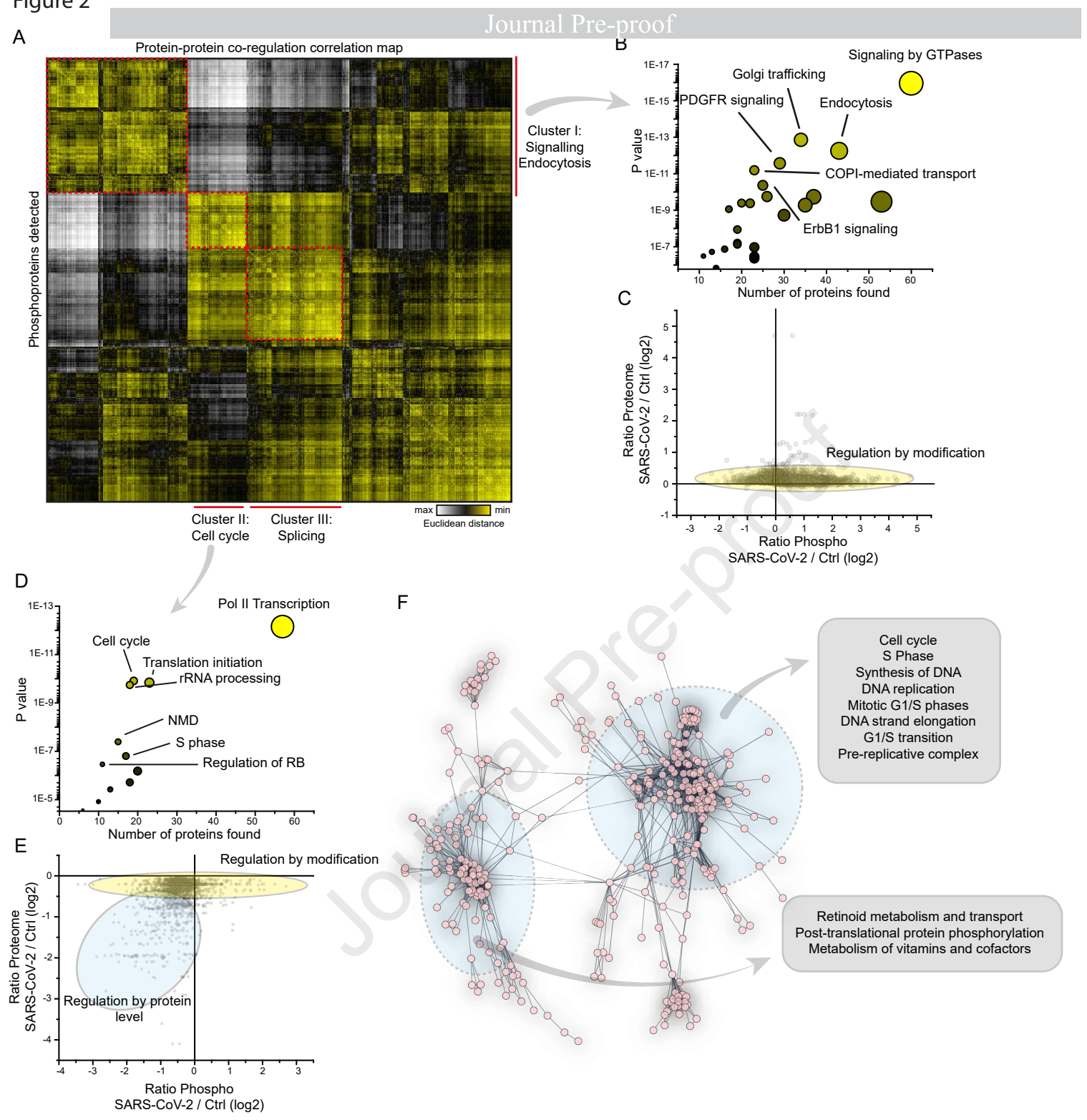


Figure 3

A

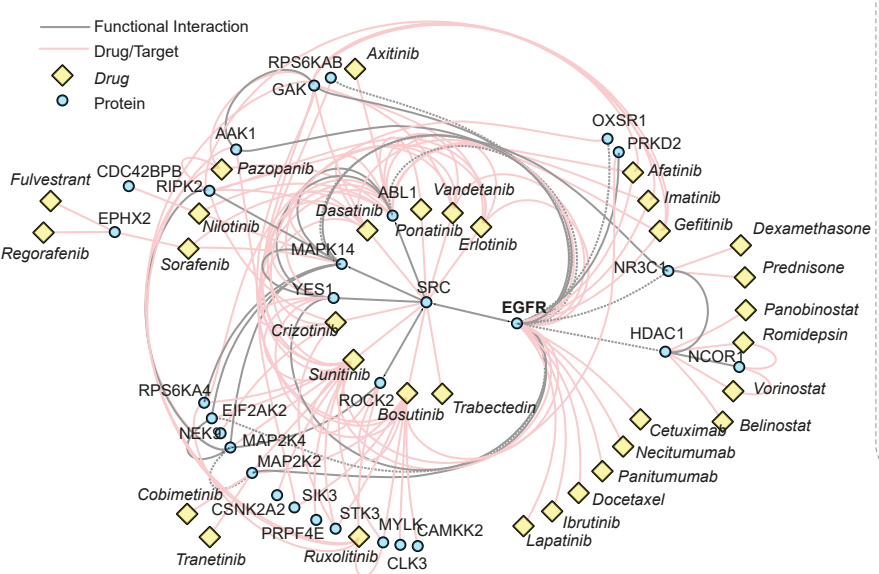

String interaction network
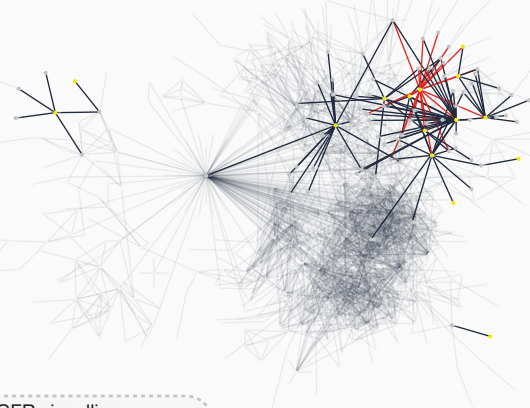

EGFR signalling

First interacting neighbour

$\diamond$ PRPF4EO $\triangle 000$ OMKK2

C

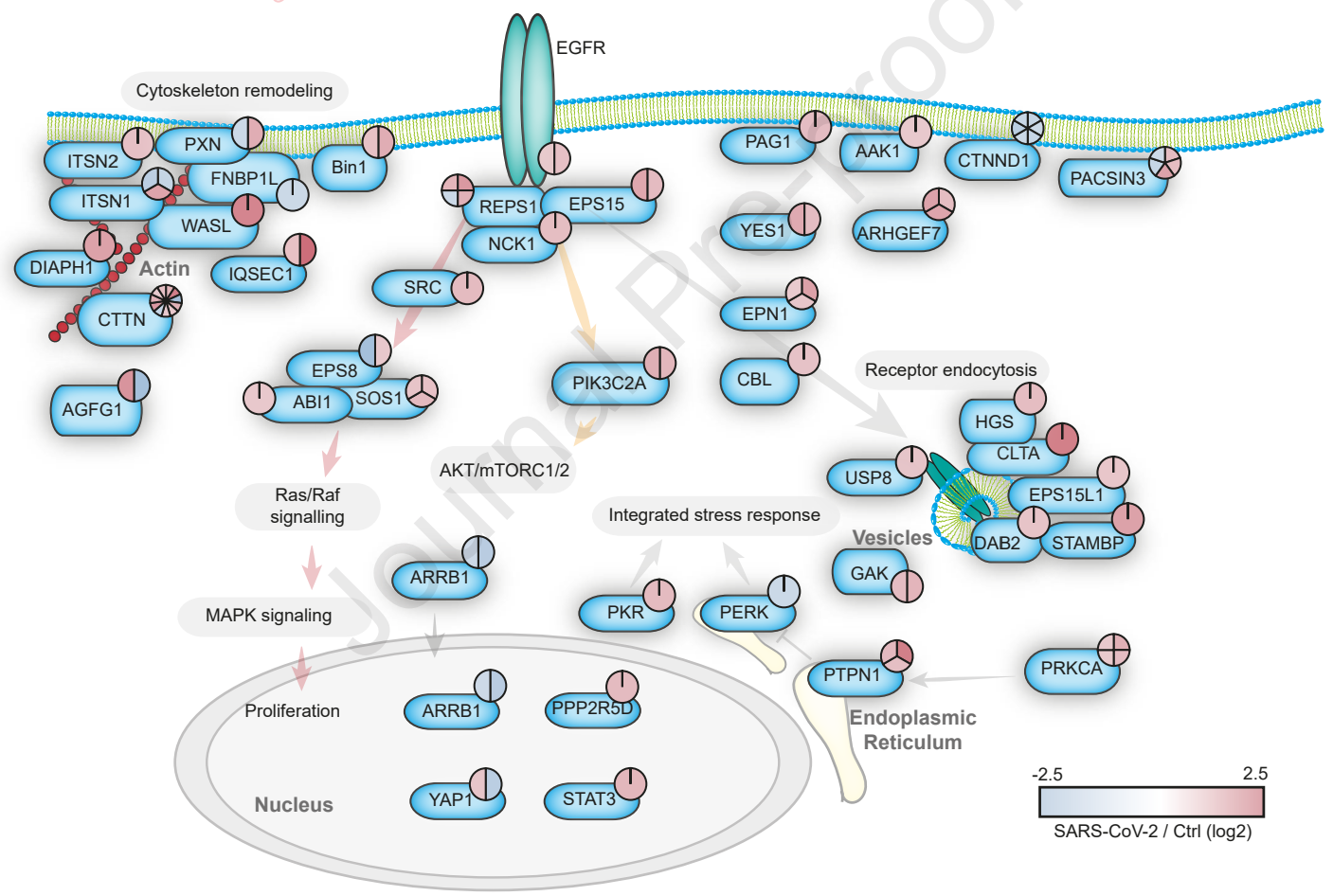


Figure 4
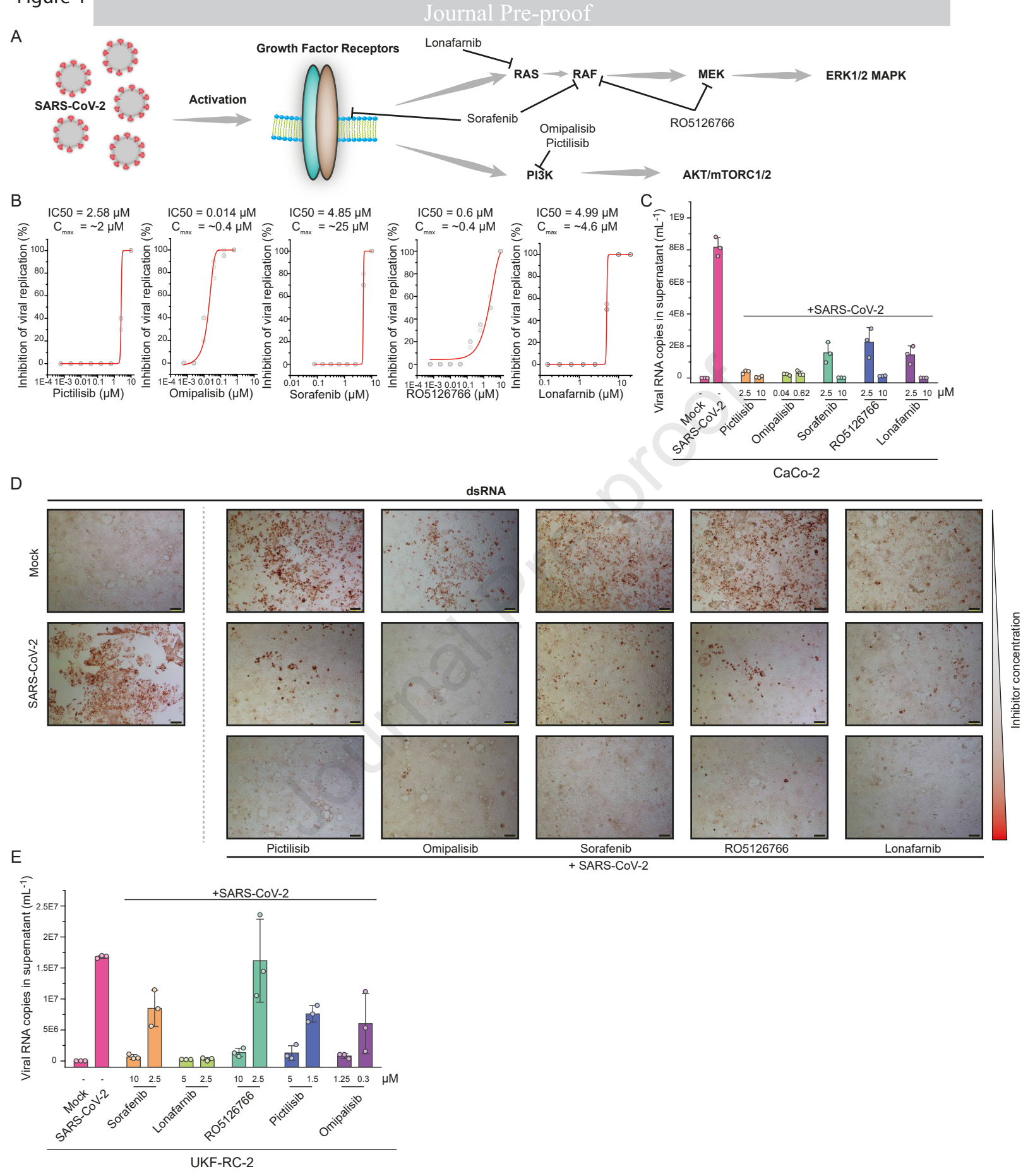
Figure 5

Journal Pre-proof

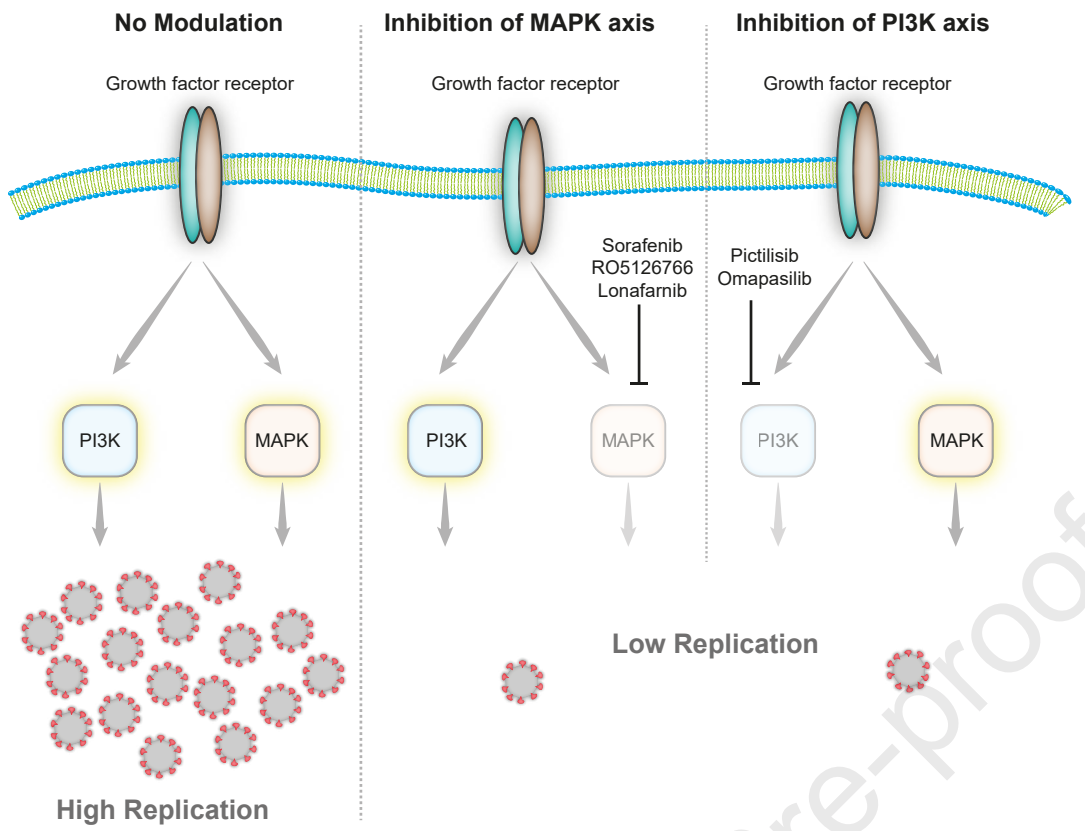




\section{Presentation of own work on manuscripts}

\section{Functional Translatome Proteomics Reveal Converging and Dose- Dependent Regulation by mTORC1 and elF2 $\alpha, 2020$, Molecular Cell}

Kevin Klann, Georg Tascher and Christian Münch

All experiments have been carried out by Kevin Klann, including cell culture work, sample preparation for mass spectrometry and partially MS instrumentation (MS instrumentation mainly by Georg Tascher). All data analysis and visualization was performed by the author. The manuscript was written by Kevin Klann and Christian Münch.

Instrument Logic Increases Identifications during Mutliplexed Translatome Measurements, 2020, Analytical Chemistry

Kevin Klann and Christian Münch

All experiments, data analysis and visualization was performed by Kevin Klann. The manuscript was written by Kevin Klann and Christian Münch.

Unbiased translation proteomics upon cell stress, 2020, Molecular and Cellular Oncology

Kevin Klann and Christian Münch

The manuscript was written by Kevin Klann and Christian Münch. 


\section{Proteomics of SARS-CoV-2-infected host cells reveals therapy targets, 2020, Nature (Co-First author)}

Denisa Bojkova, Kevin Klann, Benjamin Koch, Marek Widera, David Krause, Sandra Ciesek, Jindrich Cinatl and Christian Münch

Kevin Klann performed all proteomics experiments (including sample preparation, instrumentation and data analysis) and prepared all figures and visualizations. The first manuscript draft was written by Kevin Klann and Christian Münch, while the final manuscript was written with input from all authors. All other experiments have been carried out by co-authors.

\section{Growth factor receptor signaling inhibition prevents SARS-CoV-2 replication, 2020, Molecular Cell (Co-First author)}

Kevin Klann, Denisa Bojkova, Georg Tascher, Sandra Ciesek, Christian Münch and Jindrich Cinatl

Kevin Klann performed all proteomics experiments (including sample preparation, instrumentation and data analysis) and prepared all figures and visualizations. The first manuscript draft was written by Kevin Klann and Christian Münch, while the final manuscript was written with input from all authors. All other experiments have been carried out by co-authors. 


\section{References}

1. Blagosklonny, MV. Hsp-90-associated oncoproteins: multiple targets of geldanamycin and its analogs. Leukemia 2002;16:455-462.

2. Halliday, M, Radford, H, Sekine, $\mathrm{Y}$, et al. Partial restoration of protein synthesis rates by the small molecule ISRIB prevents neurodegeneration without pancreatic toxicity. Cell death \& disease 2015;6:e1672-e1672.

3. Hilton, A, Mizzen, L, Macintyre, G, Cheley, S, and Anderson, R. Translational Control in Murine Hepatitis Virus Infection. Journal of General Virology 1986;67:923-932.

4. Klann, K, Tascher, G, and Münch, C. Functional Translatome Proteomics Reveal Converging and Dose-Dependent Regulation by mTORC1 and elF2 $\alpha$. Molecular Cell 2020;77:913-925.e4.

5. Pópulo, H, Lopes, JM, and Soares, P. The mTOR signalling pathway in human cancer. International journal of molecular sciences 2012;13:18861918.

6. Klann, $\mathrm{K}$ and Münch, C. Unbiased translation proteomics upon cell stress. Molecular \& Cellular Oncology 2020:1763150.

7. Klann, K and Münch, C. Instrument Logic Increases Identifications during Mutliplexed Translatome Measurements. Analytical Chemistry 2020.

8. Chan, JFW, Yuan, S, Kok, KH, et al. A familial cluster of pneumonia associated with the 2019 novel coronavirus indicating person-to-person transmission: a study of a family cluster. The Lancet 2020.

9. Tang, B, Wang, X, Li, Q, et al. Estimation of the Transmission Risk of the 2019-nCoV and Its Implication for Public Health Interventions. Journal of Clinical Medicine 2020;9:462.

10. Zhu, N, Zhang, D, Wang, W, et al. A Novel Coronavirus from Patients with Pneumonia in China, 2019. New England Journal of Medicine 2020;382:727733. 
11. Bojkova, D, Klann, K, Koch, B, et al. Proteomics of SARS-CoV-2-infected host cells reveals therapy targets. Nature 2020.

12. Klann, K, Bojkova, D, Tascher, G, Ciesek, S, Münch, C, and Cinatl, J. Growth factor receptor signaling inhibition prevents SARS-CoV-2 replication. Molecular Cell 2020:2020.05.14.095661.

13. Fortelny, N, Overall, CM, Pavlidis, P, and Freue, GVC. Can we predict protein from mRNA levels? Nature 2017;547:E19-E20.

14. Sonenberg, $\mathrm{N}$ and Hinnebusch, AG. Regulation of Translation Initiation in Eukaryotes: Mechanisms and Biological Targets. Cell 2009;136:731-745.

15. Deribe, YL, Pawson, T, and Dikic, I. Post-translational modifications in signal integration. Nature Structural \& Molecular Biology 2010;17:666-672.

16. Dikic, I. Proteasomal and Autophagic Degradation Systems. Annual Review of Biochemistry 2017;86:193-224.

17. Kornitzer, D and Ciechanover, A. Modes of regulation of ubiquitin-mediated protein degradation. Journal of Cellular Physiology 2000;182:1-11.

18. Chou, A, Krukowski, K, Jopson, T, et al. Inhibition of the integrated stress response reverses cognitive deficits after traumatic brain injury. Proceedings of the National Academy of Sciences 2017;114:E6420 LP -E6426.

19. Hsieh, AC, Liu, Y, Edlind, MP, et al. The translational landscape of mTOR signalling steers cancer initiation and metastasis. Nature 2012;485:55-61.

20. Narayanan, K, Huang, C, Lokugamage, K, et al. Severe Acute Respiratory Syndrome Coronavirus nsp1 Suppresses Host Gene Expression, Including That of Type I Interferon, in Infected Cells. Journal of Virology 2008;82:4471 LP -4479.

21. DuRose, JB, Scheuner, D, Kaufman, RJ, Rothblum, LI, and Niwa, M. Phosphorylation of Eukaryotic Translation Initiation Factor $2 \alpha$ Coordinates rRNA Transcription and Translation Inhibition during Endoplasmic Reticulum Stress. Molecular and Cellular Biology 2009;29:4295 LP _4307.

22. Pakos $\square$ Zebrucka, K, Koryga, I, Mnich, K, Ljujic, M, Samali, A, and Gorman, AM. The integrated stress response. EMBO reports 2016;17:1374 LP -1395. 
23. Taniuchi, S, Miyake, M, Tsugawa, K, Oyadomari, M, and Oyadomari, S. Integrated stress response of vertebrates is regulated by four elF $2 \alpha$ kinases. Scientific Reports 2016;6:32886.

24. Dowling, RJO, Topisirovic, I, Alain, T, et al. mTORC1-mediated cell proliferation, but not cell growth, controlled by the 4E-BPs. Science (New York, N.Y.) 2010;328:1172-6.

25. Wengrod, JC and Gardner, LB. Cellular adaptation to nutrient deprivation: crosstalk between the mTORC1 and elF2 $\alpha$ signaling pathways and implications for autophagy. Cell Cycle 2015;14:2571-2577.

26. Reid, DW, Chen, Q, Tay, ASL, Shenolikar, S, and Nicchitta, CV. The Unfolded Protein Response Triggers Selective mRNA Release From the Endoplasmic Reticulum. Cell 2014;158:1362-1374.

27. Sidrauski, C, McGeachy, AM, Ingolia, NT, and Walter, P. The small molecule ISRIB reverses the effects of elF $2 \alpha$ phosphorylation on translation and stress granule assembly. eLife 2015;4. Ed. by Ron, D:e05033.

28. Thoreen, CC, Chantranupong, L, Keys, HR, Wang, T, Gray, NS, and Sabatini, DMM. A unifying model for mTORC1-mediated regulation of mRNA translation. Nature 2012;485:109-113.

29. Fiskin, E, Bionda, T, Dikic, I, and Behrends, C. Global Analysis of Host and Bacterial Ubiquitinome in Response to Salmonella Typhimurium Infection. Molecular Cell 2016;62:967-981.

30. Herhaus, L and Dikic, I. Regulation of Salmonella-host cell interactions via the ubiquitin system. International Journal of Medical Microbiology 2018;308:176-184.

31. De Leon, JA, Qiu, J, Nicolai, CJ, et al. Positive and Negative Regulation of the Master Metabolic Regulator mTORC1 by Two Families of Legionella pneumophila Effectors. Cell reports 2017;21:2031-2038.

32. Kamitani, W, Huang, C, Narayanan, K, Lokugamage, KG, and Makino, S. A two-pronged strategy to suppress host protein synthesis by SARS coronavirus Nsp1 protein. Nature Structural \& Molecular Biology 2009;16:11341140. 
33. Walsh, D. Manipulation of the host translation initiation complex elF4F by DNA viruses. Biochemical Society Transactions 2010;38:1511-1516.

34. Bai, Y, Zhou, K, and Doudna, JA. Hepatitis C virus 3'UTR regulates viral translation through direct interactions with the host translation machinery. Nucleic Acids Research 2013;41:7861-7874.

35. Balachandran, S, Roberts, PC, Brown, LE, et al. Essential Role for the dsRNA-Dependent Protein Kinase PKR in Innate Immunity to Viral Infection. Immunity 2000;13:129-141.

36. Dauber, B and Wolff, T. Activation of the Antiviral Kinase PKR and Viral Countermeasures. Viruses 2009;1:523-544.

37. Morrison, J, Laurent-Rolle, M, Maestre, AM, et al. Dengue Virus Co-opts UBR4 to Degrade STAT2 and Antagonize Type I Interferon Signaling. PLOS Pathogens 2013;9:e1003265.

38. Schepetilnikov, M, Kobayashi, K, Geldreich, A, et al. Viral factor TAV recruits TOR/S6K1 signalling to activate reinitiation after long ORF translation. The EMBO Journal 2011;30:1343-1356.

39. Reid, DW, Campos, RK, Child, JR, et al. Dengue Virus Selectively Annexes Endoplasmic Reticulum-Associated Translation Machinery as a Strategy for Co-opting Host Cell Protein Synthesis. Journal of Virology 2018;92. Ed. by Diamond, MS:e01766-17.

40. Didcock, L, Young, DF, Goodbourn, S, and Randall, RE. The V Protein of Simian Virus 5 Inhibits Interferon Signalling by Targeting STAT1 for Proteasome-Mediated Degradation. Journal of Virology 1999;73:9928 LP $-9933$.

41. Lin, R, Heylbroeck, C, Pitha, PM, and Hiscott, J. Virus-Dependent Phosphorylation of the IRF-3 Transcription Factor Regulates Nuclear Translocation, Transactivation Potential, and Proteasome-Mediated Degradation. Molecular and Cellular Biology 1998;18:2986 LP -2996.

42. Bhogaraju, S, Kalayil, S, Liu, Y, et al. Phosphoribosylation of Ubiquitin Promotes Serine Ubiquitination and Impairs Conventional Ubiquitination. Cell 2016;167:1636-1649.e13. 
43. Yángüez, E, Hunziker, A, Dobay, MP, et al. Phosphoproteomic-based kinase profiling early in influenza virus infection identifies GRK2 as antiviral drug target. Nature Communications 2018;9:3679.

44. Söderholm, S, Kainov, DE, Öhman, T, et al. Phosphoproteomics to Characterize Host Response During Influenza A Virus Infection of Human Macrophages. Molecular and Cellular Proteomics 2016;15:3203 LP -3219.

45. Bantscheff, M, Schirle, M, Sweetman, G, Rick, J, and Kuster, B. Quantitative mass spectrometry in proteomics: a critical review. Analytical and Bioanalytical Chemistry 2007;389:1017-1031.

46. Bekker-Jensen, DB, Kelstrup, CD, Batth, TS, et al. An Optimized Shotgun Strategy for the Rapid Generation of Comprehensive Human Proteomes. Cell Systems 2017;4:587-599.e4.

47. Bai, B, Tan, H, Pagala, VR, et al. Deep Profiling of Proteome and Phosphoproteome by Isobaric Labeling, Extensive Liquid Chromatography, and Mass Spectrometry. Methods in enzymology 2017;585:377-395.

48. Mertins, P, Tang, LC, Krug, K, et al. Reproducible workflow for multiplexed deep-scale proteome and phosphoproteome analysis of tumor tissues by liquid chromatography-mass spectrometry. Nature Protocols 2018;13:16321661.

49. Schwanhäusser, B, Busse, D, Li, N, et al. Global quantification of mammalian gene expression control. Nature 2011;473:337-342.

50. Dieterich, DC, Link, AJ, Graumann, J, Tirrell, DA, and Schuman, EM. Selective identification of newly synthesized proteins in mammalian cells using bioorthogonal noncanonical amino acid tagging (BONCAT). Proceedings of the National Academy of Sciences of the United States of America 2006;103:9482-7.

51. Forester, CM, Zhao, Q, Phillips, NJ, et al. Revealing nascent proteomics in signaling pathways and cell differentiation. Proceedings of the National Academy of Sciences 2018;115:2353 LP -2358. 
52. Welle, KA, Zhang, T, Hryhorenko, JR, Shen, S, Qu, J, and Ghaemmaghami, $S$. Time-resolved Analysis of Proteome Dynamics by Tandem Mass Tags and Stable Isotope Labeling in Cell Culture (TMT-SILAC) Hyperplexing. Molecular \& Cellular Proteomics 2016;15:3551-3563.

53. Kiick, KL, Saxon, E, Tirrell, DA, and Bertozzi, CR. Incorporation of azides into recombinant proteins for chemoselective modification by the Staudinger ligation. Proceedings of the National academy of Sciences 2002;99:19-24.

54. Larsson, O, Morita, M, Topisirovic, I, et al. Distinct perturbation of the translatome by the antidiabetic drug metformin. Proceedings of the National Academy of Sciences 2012;109:8977 LP -8982.

55. Sabatini, DM. mTOR and cancer: insights into a complex relationship. Nature reviews. Cancer 2006;6:729-34.

56. Grifoni, A, Sidney, J, Zhang, Y, Scheuermann, RH, Peters, B, and Sette, A. A Sequence Homology and Bioinformatic Approach Can Predict Candidate Targets for Immune Responses to SARS-CoV-2. Cell Host \& Microbe 2020;27:671-680.e2.

57. Rockx, B, Kuiken, T, Herfst, S, et al. Comparative pathogenesis of COVID19, MERS, and SARS in a nonhuman primate model. Science 2020;368:1012 LP -1015 .

58. Hoehl, S, Berger, A, Kortenbusch, M, et al. Evidence of SARS-CoV-2 Infection in Returning Travelers from Wuhan, China. New England Journal of Medicine 2020.

59. Morgenstern, B, Michaelis, M, Baer, PC, Doerr, HW, and Cinatl, J. Ribavirin and interferon- $\beta$ synergistically inhibit SARS-associated coronavirus replication in animal and human cell lines. Biochemical and Biophysical Research Communications 2005;326:905-908.

60. Marcelin, JR, Wilson, JW, Razonable, RR, Services, TMCH, and Diseases, TI. Oral ribavirin therapy for respiratory syncytial virus infections in moderately to severely immunocompromised patients. Transplant Infectious Disease 2014;16:242-250. 
61. Martin, P and Jensen, DM. Ribavirin in the treatment of chronic hepatitis C. Journal of Gastroenterology and Hepatology 2008;23:844-855.

62. Eskens, FALM, Ramos, FJ, Burger, $\mathrm{H}$, et al. Phase I Pharmacokinetic and Pharmacodynamic Study of the First-in-Class Spliceosome Inhibitor E7107 in Patients with Advanced Solid Tumors. Clinical Cancer Research 2013;19:6296 LP -6304.

63. Raez, LE, Papadopoulos, K, Ricart, AD, et al. A phase I dose-escalation trial of 2-deoxy-d-glucose alone or combined with docetaxel in patients with advanced solid tumors. Cancer Chemotherapy and Pharmacology 2013;71:523-530.

64. Wu, LS, Rower, JE, Burton, JR, et al. Population Pharmacokinetic Modeling of Plasma and Intracellular Ribavirin Concentrations in Patients with Chronic Hepatitis C Virus Infection. Antimicrobial Agents and Chemotherapy 2015;59:2179 LP -2188.

65. Eskens, FALM, Awada, A, Cutler, DL, et al. Phase I and Pharmacokinetic Study of the Oral Farnesyl Transferase Inhibitor SCH 66336 Given Twice Daily to Patients With Advanced Solid Tumors. Journal of Clinical Oncology 2001;19:1167-1175.

66. Munster, P, Aggarwal, R, Hong, D, et al. First-in-Human Phase I Study of GSK2126458, an Oral Pan-Class I Phosphatidylinositol-3-Kinase Inhibitor, in Patients with Advanced Solid Tumor Malignancies. Clinical Cancer Research 2016;22:1932 LP -1939.

67. Martinez-Garcia, M, Banerji, U, Albanell, J, et al. First-in-Human, Phase I Dose-Escalation Study of the Safety, Pharmacokinetics, and Pharmacodynamics of R05126766, a First-in-Class Dual MEK/RAF Inhibitor in Patients with Solid Tumors. Clinical Cancer Research 2012;18:4806 LP -4819.

68. Kindrachuk, J, Ork, B, Hart, BJ, et al. Antiviral Potential of ERK/MAPK and PI3K/AKT/mTOR Signaling Modulation for Middle East Respiratory Syndrome Coronavirus Infection as Identified by Temporal Kinome Analysis. Antimicrobial Agents and Chemotherapy 2015;59:1088-1099. 
69. Hung, IFN, Lung, KC, Tso, EYK, et al. Triple combination of interferon beta1b, lopinavir\&\#x2013;ritonavir, and ribavirin in the treatment of patients admitted to hospital with COVID-19: an open-label, randomised, phase 2 trial. The Lancet 2020;395:1695-1704.

70. Moleculin Biotech. Tackling Coronavirus with WP1122. 2020. URL: https: //www.moleculin.com/covid-19/.

71. Bagert, JD, Xie, YJ, Sweredoski, MJ, et al. Quantitative, Time-Resolved Proteomic Analysis by Combining Bioorthogonal Noncanonical Amino Acid Tagging and Pulsed Stable Isotope Labeling by Amino Acids in Cell Culture. Molecular \& Cellular Proteomics 2014;13:1352 LP -1358.

72. Chen, K, Hu, Z, Xia, Z, Zhao, D, Li, W, and Tyler, JK. The overlooked fact: fundamental need for spike-in control for virtually all genome-wide analyses. Molecular and cellular biology 2016;36:662-667.

73. McGlincy, NJ and Ingolia, NT. Transcriptome-wide measurement of translation by ribosome profiling. Methods (San Diego, Calif.) 2017;126:112-129.

74. Jaafar, ZA and Kieft, JS. Viral RNA structure-based strategies to manipulate translation. Nature Reviews Microbiology 2019;17:110-123.

75. Castello, A, Fischer, B, Eichelbaum, K, et al. Insights into RNA Biology from an Atlas of Mammalian mRNA-Binding Proteins. Cell 2012;149:1393-1406.

76. Galban, S, Kuwano, Y, Pullmann, R, et al. RNA-Binding Proteins HuR and PTB Promote the Translation of Hypoxia-Inducible Factor $1 \alpha$. Molecular and Cellular Biology 2008;28:93-107.

77. Shi, Z and Barna, M. Translating the Genome in Time and Space: Specialized Ribosomes, RNA Regulons, and RNA-Binding Proteins. Annual Review of Cell and Developmental Biology 2015;31. PMID: 26443190:31-54.

78. Zhang, Y, Kwok-Shing Ng, P, Kucherlapati, M, et al. A Pan-Cancer Proteogenomic Atlas of PI3K/AKT/mTOR Pathway Alterations. Cancer Cell 2017;31:820-832.e3. 
79. Wei, F, Zhang, Y, Geng, L, Zhang, P, Wang, G, and Liu, Y. mTOR Inhibition Induces EGFR Feedback Activation in Association with Its Resistance to Human Pancreatic Cancer. International Journal of Molecular Sciences 2015;16:3267-3282.

80. Sun, SY, Rosenberg, LM, Wang, X, et al. Activation of Akt and elF4E Survival Pathways by Rapamycin-Mediated Mammalian Target of Rapamycin Inhibition. Cancer Research 2005;65:7052-7058.

81. Chiarini, F, Evangelisti, C, Lattanzi, G, McCubrey, JA, and Martelli, AM. Advances in understanding the mechanisms of evasive and innate resistance to mTOR inhibition in cancer cells. Biochimica et Biophysica Acta (BBA) Molecular Cell Research 2019;1866:1322-1337.

82. Costa-Mattioli, M and Walter, P. The integrated stress response: From mechanism to disease. Science 2020;368.

83. Rabouw, HH, Langereis, MA, Anand, AA, et al. Small molecule ISRIB suppresses the integrated stress response within a defined window of activation. Proceedings of the National Academy of Sciences 2019;116:2097-2102.

84. Young-Baird, SK, Lourenço, MB, Elder, MK, Klann, E, Liebau, S, and Dever, TE. Suppression of MEHMO Syndrome Mutation in elF2 by Small Molecule ISRIB. Molecular Cell 2020;77:875-886.e7.

85. Zhu, PJ, Khatiwada, S, Cui, Y, et al. Activation of the ISR mediates the behavioral and neurophysiological abnormalities in Down syndrome. Science 2019;366:843-849.

86. Wong, YL, LeBon, L, Edalji, R, Lim, HB, Sun, C, and Sidrauski, C. The small molecule ISRIB rescues the stability and activity of Vanishing White Matter Disease elF2B mutant complexes. eLife 2018;7. Ed. by Hinnebusch, AG:e32733.

87. Jourdan, SS, Osorio, F, and Hiscox, JA. An interactome map of the nucleocapsid protein from a highly pathogenic $\mathrm{N}$ orth $\mathrm{A}$ merican porcine reproductive and respiratory syndrome virus strain generated using SILAC based quantitative proteomics. Proteomics 2012;12:1015-1023. 
88. Song, T, Fang, L, Wang, D, et al. Quantitative interactome reveals that porcine reproductive and respiratory syndrome virus nonstructural protein 2 forms a complex with viral nucleocapsid protein and cellular vimentin. Journal of proteomics 2016;142:70-81.

89. Emmott, E, Munday, D, Bickerton, E, et al. The cellular interactome of the coronavirus infectious bronchitis virus nucleocapsid protein and functional implications for virus biology. Journal of virology 2013;87:9486-9500.

90. Cretu, C, Agrawal, AA, Cook, A, et al. Structural Basis of Splicing Modulation by Antitumor Macrolide Compounds. Molecular Cell 2018;70:265-273.e8.

91. Beerli, C, Yakimovich, A, Kilcher, S, et al. Vaccinia virus hijacks EGFR signalling to enhance virus spread through rapid and directed infected cell motility. Nature Microbiology 2019;4:216-225.

92. Lupberger, J, Zeisel, MB, Xiao, F, et al. EGFR and EphA2 are host factors for hepatitis $C$ virus entry and possible targets for antiviral therapy. Nature Medicine 2011;17:589-595.

93. Venkataraman, T, Coleman, C, and Frieman, M. Overactive EGFR Signaling Leads to Increased Fibrosis After SARS-CoV Infection. Journal of Virology 2017:JVI.00182-17.

94. Kung, CP, Meckes, DG, and Raab-Traub, N. Epstein-Barr Virus LMP1 Activates EGFR, STAT3, and ERK through Effects on PKC $\delta$. Journal of Virology 2011;85:4399 LP -4408.

95. Eierhoff, T, Hrincius, ER, Rescher, U, Ludwig, S, and Ehrhardt, C. The epidermal growth factor receptor (EGFR) promotes uptake of influenza $A$ viruses (IAV) into host cells. PLoS pathogens 2010;6:e1001099-e1001099.

96. Yarden, Y. The EGFR family and its ligands in human cancer: signalling mechanisms and therapeutic opportunities. European Journal of Cancer 2001;37:3-8.

97. Fan, QW, Cheng, C, Knight, ZA, et al. EGFR signals to mTOR through PKC and independently of Akt in glioma. Science signaling 2009;2:ra4. 
98. Wilhelm, S, Carter, C, Lynch, M, et al. Discovery and development of sorafenib: a multikinase inhibitor for treating cancer. Nature Reviews Drug Discovery 2006;5:835-844.

99. Dowell, J, Minna, JD, and Kirkpatrick, P. Erlotinib hydrochloride. Nature Reviews Drug Discovery 2005;4:13-14. 


\section{Acknowledgements}

I especially thank Christian Münch for his outstanding mentorship. Thank you for listening and discussing new project ideas and always supporting our work in the lab. Working in your lab makes me a better scientist every day. I want to thank all members of the Münch Lab and the quantitative MS unit, you are all truly great colleagues, making work enjoyable. Thank you. Furthermore, I want to thank Georg Tascher in particular, without you I would not have a fraction of the MS knowledge I needed for this work. Special thanks to all advisors of my thesis through the years, Tobias Schmid, Christian Brandts and Ivan Dikic. At last, I would like to thank all colleagues and collaborators inside and outside of the IBC2. It's a pleasure working with you all. 


\section{Curriculum Vitae}




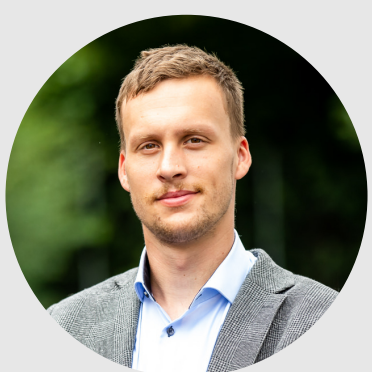

Kevin Klann PhD Candidate

(i) 07 June 1991

هiesenweg 5, 65760 Eschborn

(\%) +491772452312

(7) linkedin.com/in/kevin-klann841719130

@ klann@em.uni-frankfurt.de

\section{About me}

Creative scientist interested in systems biology of the proteome. I am currently focusing on the effect of proteostasis pertubation on the dynamics of cellular protein networks. Therefore, I am using a variety of mass spectrometry based proteomic tools and sophisticated bioinformatic analysis to drive the big picture of cell biology. Thus, I am highly experienced with multiplexed proteomic workflows and data analysis, multi-omics integration and always ready to learn new things to solve a biological question.

\section{Skills}

Systems biology

\begin{tabular}{l} 
Problem solving \\
\hline Proteomics \\
\hline Statistics
\end{tabular}

Python $\star 4$ Matlab $\star 2$ Ribosome Profiling $\star 3 q P C R \star 4 P T M$ profiling $\star 4$ Immunoprecipitation $* 3$ Microscopy $\star 3$ Protein purification $\star 3$ Cell culture $\star 4$ Broad repertoire of cell biological methods $\star 5$

$\left.{ }^{*}\right)[$ The skill scale is from 0 (Fundamental Awareness) to 6 (Expert).]

\section{Education}

since 2017 Ph.D. candidate in Biochemistry Goethe University, Frankfurt "Proteome dynamics upon stress and disease" with Dr. C. Münch at Institute for Biochemistry II

2014-2016 M.Sc. Molecular biology (Grade: 1.3) Ruprechts-Karl-Universität Heidelberg Thesis: "Characterization of translational regulation and cotranslational complex formation of the yeast fatty acid synthase" with Prof. B. Bukau at ZMBH

2011-2014 B.Sc. Biosciences (Grade: 1.6) Goethe University, Frankfurt Thesis: "Regulation of E3-Ligase CHIP in Solanum lycopersicum" with Prof. E. Schleiff

\section{Publications}

2020 Growth factor receptor signaling inhibition prevents SARS-CoV-2 replication Molecular Cel K. Klann*,D. Bojkova*,G. Tascher, S. Ciesek, C. Münch and J. Cinatl (*equally contributed)

2020 Instrument logic increases identifications during multiplexed translatome measurements Analytical Chemistry K. Klann and C. Münch

$2020 \quad$ Unbiased translation proteomics upon cell stress Molecular and Cellular Oncology

K. Klann and C. Münch

2020 Proteomics of SARS-CoV-2 infected host cells reveals therapy targets Nature

D. Bojkova*, K. Klann*, B. Koch*, M. Widera, D. Krause, S. Ciesek, J. Cinatl and C. Münch (* equally contributed)

2020 Serine-dependent redox homeostasis regulates glioblastoma cell survival British Jorunal of Cance A.L. Engel, N.I. Lorenz, K. Klann, C. Münch, C. Depner, J.P. Steinbach, M.W. Ronellenfitsch and A.L. Luger

2019 Functional translatome proteomics reveal converging and dosedependent regulation of MTORC1 and eIF2a Molecular Cell K. Klann, G. Tascher and C. Münch

2019 Loss of the selective autophagy receptor p62 impairs murine leukemia progression and mitophagy Blood T.D. Nguyen, S. Shaid, O. Vakhrusheva, S.E. Koschade, K. Klann, M. Thölken, F. Baker, J. Zhang, T. Oellerich, D. Sürün, A. Derlet, I. Haberbosch, S. Eimer, H.D. Osiewacz, C. Behrends, C. Münch, I. Dikic and C.H. Brandts

2018 Cotranslational assembly of protein complexes in eukaryotes revealed by ribosome profiling

Nature A. Shiber, K. Döring, U. Friedrich, K. Klann, D. Merker, M. Zedan, F. Tippmann, G. Kramer and B. Bukau

\section{Awards}

$2020 \quad$ Tandem Mass Tag Award by ThermoFisher

2019 FEBS Poster Prize at EMBO Proteostasis Workshop

\section{Practical experience}

$2016 \quad$ Master Thesis at ZMBH, Heidelberg (Bukau lab)

2015-2016 Research assistant at MPI for medical research, Heidelberg (Cryle lab)

2015-2016 Several research practicals during Molecular Biology M.sc. program

Date, Signature 
Schriftliche Erklärung 


\section{Schriftliche Erklärung}

Ich erkläre ehrenwörtlich, dass ich die dem Fachbereich Medizin der Johann Wolfgang Goethe-Universität Frankfurt am Main zur Promotionsprüfung eingereichte Dissertation mit dem Titel

Proteome dynamics upon proteotoxic stress and disease

in der/dem Institut für Biochemie II unter Betreuung und Anleitung von Dr. Christian Münch mit Unterstützung durch Prof. Dr. Christian Brandts ohne sonstige Hilfe selbst durchgeführt und bei der Abfassung der Arbeit keine anderen als die in der Dissertation angeführten Hilfsmittel benutzt habe. Darüber hinaus versichere ich, nicht die Hilfe einer kommerziellen Promotionsvermittlung in Anspruch genommen zu haben.

Ich habe bisher an keiner in- oder ausländischen Universität ein Gesuch um Zulassung zur Promotion eingereicht*. Die vorliegende Arbeit wurde bisher nicht als Dissertation eingereicht.

Vorliegende Ergebnisse der Arbeit wurden (oder werden) in folgendem Publikationsorgan veröffentlicht:

1. Klann K, Münch C. Instrument Logic Increases Identifications during Mutliplexed Translatome Measurements. Analytical Chemistry. 92, 12, 8041-8045, 2020

2. Klann K, Tascher G, Münch C. Functional Translatome Proteomics Reveal Converging and Dose-Dependent Regulation by mTORC1 and eIF2 $\alpha$. Molecular Cell. ;77(4):913--925.e4., 2020

3. Bojkova D, Klann K, Koch B, Widera M, Krause D, Ciesek S, Cinatl J, Münch C. Proteomics of SARSCoV-2-infected host cells reveals therapy targets. Nature. 583, 469-472, 2020

4. Klann, K., Bojkova, D., Tascher, G., Ciesek, S., Münch, C., and Cinatl, J. Growth factor receptor signaling inhibition prevents SARS-CoV-2 replication. Molecular Cell, 2020 (in press)

5. Klann K, Münch C. Unbiased translation proteomics upon cell stress. Molecular and Cellular Oncology. 7:4, 1763150,2020

(Ort, Datum)

*) im Falle des Nichtzutreffens entfernen
(Unterschrift) 